IRB-TH-13/00

\title{
BLM scale for the pion transition form factor
}

\author{
B. Melid \\ Institut für Physik, Universität Mainz, \\ D-55099 Mainz, Germany and \\ Institut für Theoretische Physik, Universität Würzburg, \\ D-97074 Würzburg, Germany \\ B. Nižići and K. Passekf \\ Theoretical Physics Division, Rudjer Bošković Institute, \\ P.O. Box 180, HR-10002 Zagreb, Croatia
}

(Dated: April 29, 2019)

\begin{abstract}
The NLO Brodsky-Lepage-Mackenzie (BLM) scale for the pion transition form factor has been determined. To achieve that, a consistent calculation up to $n_{f}$-proportional NNLO contributions to both the hard-scattering amplitude and the perturbatively calculable part of the pion distribution amplitude has been performed. By combining and matching the results obtained for these two amplitudes, a proper cancellation of collinear singularities has been established and the $\gamma_{5}$ ambiguity problem (related to the use of the dimensional regularization method) has been resolved by using the naive- $\gamma_{5}$ as well as the 't Hooft-Veltman (HV) schemes. It has been demonstrated that the prediction for the pion transition form factor is independent of the factorization scale $\mu_{F}^{2}$ at every order in the strong coupling constant, making it possible to use the simplest choice $\mu_{F}^{2}=Q^{2}$ at the intermediate steps of the calculation. Assuming the pion asymptotic distribution amplitude and working in the $\overline{M S}$ scheme, we have found the BLM scale to be $\mu_{R}^{2}=\mu_{B L M}^{2} \approx Q^{2} / 9$. Based on the same distribution, the complete NLO prediction for the pion transition form factor has been calculated in the $\alpha_{V}$ definition of the QCD coupling renormalized at $\mu_{R}^{2}=\mu_{V}^{2}=e^{5 / 3} \mu_{B L M}^{2} \approx Q^{2} / 2$. It is in good agreement with the presently available experimental data.
\end{abstract}

PACS numbers: 13.40.Gp,12.38.Bx,13.60.Le,11.15.Me,13.40.Hq

\section{INTRODUCTION}

The pion transition form factor plays a crucial role in testing QCD predictions for exclusive processes. It appears in the amplitude that relates two, in general virtual, photons with the lightest hadron, the pion, $\gamma^{*} \gamma^{*} \rightarrow \pi^{0}$. Historically, this process attracted much interest since the axial anomaly [1] fixes the value of the form factor when both virtualities of the photons are zero $\left(\gamma \gamma \rightarrow \pi^{0}\right)$. For large virtualities of the photons (or at least for one of them) perturbative QCD (pQCD) is applicable [2]. A specific feature of this process is that the leading-order ( $\mathrm{LO})$ prediction is zeroth order in the QCD coupling constant, and one expects that pQCD for this process may work at accessible values of spacelike photon virtualities [3]. Experimentally, the most favorable situation is when one of the photons is real $\left(\gamma^{*} \gamma \rightarrow \pi^{0}\right)$.

The framework for analyzing exclusive processes at large-momentum transfer within the context of pQCD was initiated and developed in the late seventies [2, 4. It was demonstrated to all orders in perturbation the-

\footnotetext{
*Alexander von Humboldt Fellow. On leave of absence from the Budjer Bošković Institute, Zagreb, Croatia.; Electronic address: melic@thphys.irb.hr

† Electronic address: nizic@thphys.irb.hn

$\ddagger$ Electronic address: passek@thphys.irb.hn
}

ory that exclusive amplitudes at large-momentum transfer factorize into a convolution of a process-dependent and perturbatively calculable hard-scattering amplitude, with a process-independent distribution amplitude (DA), one for each hadron involved in the amplitude. Whereas the DA is intrinsically nonperturbative and its form is determined by some nonperturbative methods, the DA evolution is subject to a perturbative treatment. In the standard hard-scattering approach (sHSA), a hadron is regarded as consisting only of valence Fock states, transverse quark momenta are neglected (collinear approximation) as well as quark masses.

Although the LO predictions in the sHSA (as well as in the modified hard-scattering approach (mHSA) in which the collinear approximation is abandoned [5]) have been obtained for many exclusive processes, only a few processes have been analyzed at the next-to-leading order (NLO): the pion electromagnetic form factor $\sqrt{6}$, 7 , 8, the pion transition form factor [7, 9, 10 (and [11] in the mHSA), and the process $\gamma \gamma \rightarrow M \bar{M}(M=\pi, K)$ [12].

It is well known that, unlike in QED, one cannot rely upon the LO predictions in pQCD (the expansion parameter, i.e., the running coupling constant is rather large at current energies), and that higher-order corrections are important. The size of the NLO correction as well as the size of the expansion parameter, i.e. the QCD running coupling constant, can serve as sensible indicators of the convergence of the expansion. However, as the 
truncation of the perturbative expansion at any finite order causes the residual dependence of the prediction on the choice of the renormalization scale and scheme, these choices introduce an ambiguity in the interpretation of the finite-order perturbative prediction. In general, including higher-order corrections has a stabilizing effect (see [8], for illustration) reducing the dependence of the predictions on the schemes and scales (since the all-order prediction is independent of the scheme and scale choice). However, to asses the convergence of the perturbative expansion, it is necessary not only to extend the calculation beyond the $\mathrm{LO}$ (which is a very demanding task in many cases), but also to optimize the choices of the scale and scheme according to some sensible criteria.

In the Brodsky-Lepage-Mackenzie (BLM) procedure [13, 14], all vacuum-polarization effects from the QCD $\beta$-function are resummed into the running coupling constant. Since the coefficients $\beta_{0}, \beta_{1}, \cdots$ are functions of $n_{f}$ (number of flavors), according to BLM procedure, the renormalization scale best suited to a particular process in a given order can be, in practice, determined by computing vacuum-polarization insertions in the diagrams of that order, and by setting the scale demanding that $n_{f}$-proportional terms should vanish. The renormalization scales in the BLM method are physical in the sense that they reflect the mean virtuality of the gluon propagators and the important advantage of this method is "pre-summing" the large $\left(\beta_{0} \alpha_{S}\right)^{n}$ terms, i.e., the infrared renormalons associated with the coupling constant renormalization (15] and references therein).

The optimization of the renormalization scale and scheme for exclusive processes by employing the BLM scale fixing was elaborated in Ref. 15]. It was stated that exclusive processes are especially sensitive to the choice of the renormalization scale for the underlying hardscattering amplitude and since each external momentum entering an exclusive reaction is partitioned among many propagators of the underlying hard-scattering amplitude, the physical scales that control these processes are inevitably much softer than the overall momentum transfer. The BLM method was applied to the pion electromagnetic form factor and the $\gamma \gamma \rightarrow \pi^{+} \pi^{-}$process. For the pion transition form factor, the size of the BLM scale was only assumed (taken the same as for the pion electromagnetic form factor). Since the LO prediction for the pion transition form factor is zeroth order in the QCD coupling constant, the NLO corrections $[7,9,10$ represent leading QCD corrections and the vacuum polarization contributions appearing at the next-to-next-toleading order (NNLO) are necessary for determining the BLM scale for this process.

The purpose of this work is to determine the BLM scale for the pion transition form factor, i.e., for the $\gamma^{*} \gamma \rightarrow \pi$ process. Although, the structure of the process is simple, the calculation of higher-order corrections to the hardscattering amplitude is complicated by the $\gamma_{5}$ ambiguity, which appears when using dimensional regularization. In our calculation we use dimensional regulariza- tion in $D=4-2 \epsilon$ dimensions to regularize both ultraviolet (UV) and collinear singularities. We have obtained the LO, NLO, and $n_{f}$-proportional NNLO terms for the hard-scattering amplitude using the Feynman gauge and modified minimal-subtraction scheme $(\overline{\mathrm{MS}})$ for which a suitable compact form has been adopted. In order to correctly subtract the collinear singularities and also to verify the right choice of the $\gamma_{5}$ prescription, we have also determined the LO, NLO, and $n_{f}$-proportional NNLO terms of the perturbatively calculable (evolutional) part of the distribution amplitude. The $\gamma_{5}$ ambiguity present in the calculation of the hard-scattering part has been resolved by combining and matching the results for the hard-scattering amplitude with the results for the distribution amplitude part. The proper cancellation of singularities has been established and the $\gamma_{5}$ problem has been resolved using both the so-called naive- $\gamma_{5}$ [16 and the 't Hooft-Veltman (HV) schemes [17, 18]. It has been demonstrated that the prediction for the pion transition form factor is independent of the factorization scale $\mu_{F}^{2}$ at every order in strong coupling constant. Finally, we have been able to justify the natural choice $\mu_{F}^{2}=Q^{2}$ for the factorization scale and to determine the renormalization scale using the BLM scale setting method.

The plan of the paper is as follows. Section II is devoted to some preliminary considerations. In Sec. III the calculational procedure is briefly outlined. In Sec. IV the LO, NLO, and the $n_{f}$-proportional NNLO unrenormalized contribution to both the hard-scattering amplitude and the perturbatively calculable part of the pion distribution amplitude are obtained. Renormalization of the UV divergences and factorization of the collinear divergences present in the hard-scattering and the pion distribution amplitude are performed in Sec. V. The results for both amplitudes are obtained in the naive- $\gamma_{5}$ as well as the HV scheme. The complete leading-twist analytical expression for the pion transition form factor up to $n_{f^{-}}$ proportional NNLO terms is obtained in Sec. VI. Section VII is devoted to determining the BLM scale for the pion transition form factor based on which the complete NLO numerical predictions are then obtained in the $\overline{M S}$ and $\alpha_{V}$ renormalization schemes. The concluding remarks are given in Sec. VII. The $\gamma_{5}$ problem is addressed in detail in Appendix A. In Appendix B the Feynman rules for the perturbatively calculable part of the distribution amplitude are derived. Finally, in Appendix C, we clarify some often obscured points on the coupling constant renormalization and justify our renormalization convention.

\section{PRELIMINARIES}

The pion transition form factor $F_{\gamma * \gamma \pi}\left(Q^{2}\right)$ for a pseudoscalar meson $\pi^{0}$ is defined in terms of the amplitude $\Gamma^{\mu \nu}$ for $\gamma^{*}(q, \mu)+\gamma(k, \nu) \rightarrow \pi(P)$, as

$$
\Gamma^{\mu \nu}=i e^{2} F_{\gamma^{*} \gamma \pi}\left(Q^{2}\right) \epsilon^{\mu \nu \alpha \beta} P_{\alpha} q_{\beta} .
$$




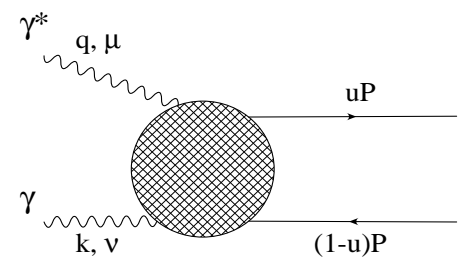

FIG. 1: Feynman diagram describing the $\gamma^{*} \gamma \rightarrow q \bar{q}$ amplitude in terms of which the hard-scattering amplitude for the $\gamma^{*} \gamma \rightarrow$ $\pi$ transition is obtained.

For large-momentum transfer $Q^{2}=-q^{2}$, the form factor can be represented [2, 国 as a convolution

$$
F_{\gamma^{*} \gamma \pi}\left(Q^{2}\right)=\Phi^{*}\left(x, \mu_{F}^{2}\right) \otimes T_{H}\left(x, Q^{2}, \mu_{F}^{2}\right),
$$

where $\otimes$ stands for the usual convolution symbol defined by

$$
A(z) \otimes B(z)=\int_{0}^{1} d z A(z) B(z) .
$$

In Eq. (2.2), the function $T_{H}\left(x, Q^{2}, \mu_{F}^{2}\right)$ is the hardscattering amplitude for producing a collinear $q \bar{q}$ pair from the initial photon pair; $\Phi^{*}\left(x, \mu_{F}^{2}\right)$ is the pion distribution amplitude representing the amplitude for the final state $q \bar{q}$ to fuse into a pion, i.e., the probability amplitude for finding the valence $q \bar{q}$ Fock state in the final pion with the constituents carrying fractions $x$ and $(1-x)$ of the meson's total momentum $P ; \mu_{F}^{2}$ is the factorization (or separation) scale at which soft and hard physics factorize.

The hard-scattering amplitude $T_{H}$ is obtained by evaluating the $\gamma^{*} \gamma \rightarrow q \bar{q}$ amplitude, which is described by the Feynman diagrams in Fig. 11, with massless on-shell quarks collinear with outgoing meson. By definition, $T_{H}$ is free of collinear singularities and has a well-defined expansion in $\alpha_{S}\left(\mu_{R}^{2}\right)$, with $\mu_{R}^{2}$ being the renormalization (or coupling constant) scale of the hard-scattering amplitude. Thus, one can write

$$
\begin{aligned}
T_{H}\left(x, Q^{2}, \mu_{F}^{2}\right) & \\
= & T_{H}^{(0)}\left(x, Q^{2}\right)+\frac{\alpha_{S}\left(\mu_{R}^{2}\right)}{4 \pi} T_{H}^{(1)}\left(x, Q^{2}, \mu_{F}^{2}\right) \\
& \quad+\frac{\alpha_{S}^{2}\left(\mu_{R}^{2}\right)}{(4 \pi)^{2}} T_{H}^{(2)}\left(x, Q^{2}, \mu_{F}^{2}, \mu_{R}^{2}\right)+\cdots .
\end{aligned}
$$

Although the function $\Phi\left(x, \mu_{F}^{2}\right)$ is intrinsically nonperturbative (containing the effects of confinement, nonperturbative interactions, and meson bound-state dynamics), it satisfies an evolution equation of the form

$$
\mu_{F}^{2} \frac{\partial}{\partial \mu_{F}^{2}} \Phi\left(x, \mu_{F}^{2}\right)=V\left(x, u, \alpha_{S}\left(\mu_{F}^{2}\right)\right) \otimes \Phi\left(u, \mu_{F}^{2}\right),
$$

where $V\left(x, u, \alpha_{S}\left(\mu_{F}^{2}\right)\right)$ is the perturbatively calculable evolution kernel

$$
\begin{aligned}
& V\left(x, u, \alpha_{S}\left(\mu_{F}^{2}\right)\right) \\
& \quad=\frac{\alpha_{S}\left(\mu_{F}^{2}\right)}{4 \pi} V_{1}(x, u)+\frac{\alpha_{S}^{2}\left(\mu_{F}^{2}\right)}{(4 \pi)^{2}} V_{2}(x, u)+\cdots .
\end{aligned}
$$

If the distribution amplitude $\Phi\left(x, \mu_{0}^{2}\right)$ is determined at an initial momentum scale $\mu_{0}^{2}$ (using some nonperturbative methods), then the differential-integral evolution equation (2.5) can be integrated using the moment method to give $\Phi\left(x, \mu_{F}^{2}\right)$ at any momentum scale $\mu_{F}^{2}>\mu_{0}^{2}$. The one- 22] and two-loop [19, 20, 21 corrections to the evolution kernel are known, but because of the complicated structure of the two-loop corrections, it was possible to obtain numerically only the first few moments of the evolution kernel [22. However, based on the conformal spin expansion, the conformal Ward identities, and the conformal consistency relation, the complete analytical form of the NLO solution of the evolution equation (2.5) has been obtained in Ref. [23].

Instead of using $\Phi\left(x, \mu_{F}^{2}\right)$, one often introduces the distribution amplitude $\phi\left(x, \mu_{F}^{2}\right)$ normalized to unity

$$
\int_{0}^{1} d x \phi\left(x, \mu_{F}^{2}\right)=1
$$

and related to $\Phi\left(x, \mu_{F}^{2}\right)$ by

$$
\Phi\left(x, \mu_{F}^{2}\right)=N_{\Phi} \phi\left(x, \mu_{F}^{2}\right),
$$

where

$$
N_{\Phi}=\frac{f_{\pi}}{2 \sqrt{2 N_{c}}}
$$

is the normalization constant imposed by the leptonic $\pi^{+} \rightarrow \mu^{+} \nu_{\mu}$ decay, $f_{\pi}=0.131 \mathrm{GeV}$ is the pion decay constant, and $N_{c}(=3)$ is the number of QCD colors.

It is convenient to expand the distribution amplitude $\phi\left(x, \mu_{0}^{2}\right)$ (determined at the initial scale $\mu_{0}^{2}$ ) in terms of the Gegenbauer polynomials $C_{n}^{3 / 2}(2 x-1)$, representing the eigenfunctions of the LO evolution kernel $V_{1}$ :

$$
\phi\left(x, \mu_{0}^{2}\right)=6 x(1-x) \sum_{n=0}^{\infty}{ }^{\prime} B_{n} C_{n}^{3 / 2}(2 x-1) .
$$

The nonperturbative input is now contained in the $B_{n}$ coefficients and $\sum^{\prime}$ denotes the sum over even indices. The DA (2.10), when evoluted to the scale $\mu_{F}^{2}$, is represented by the perturbative expansion

$$
\phi\left(x, \mu_{F}^{2}\right)=\phi^{L O}\left(x, \mu_{F}^{2}\right)+\frac{\alpha_{S}\left(\mu_{F}^{2}\right)}{4 \pi} \phi^{N L O}\left(x, \mu_{F}^{2}\right)+\cdots,
$$

where

$$
\begin{gathered}
\phi^{L O}\left(x, \mu_{F}^{2}\right)=6 x(1-x) \sum_{n=0}^{\infty}{ }^{\prime} B_{n}^{L O}\left(\mu_{F}^{2}\right) C_{n}^{3 / 2}(2 x-1), \\
\phi^{N L O}\left(x, \mu_{F}^{2}\right)=6 x(1-x) \sum_{k=2}^{\infty}{ }^{\prime} B_{k}^{N L O}\left(\mu_{F}^{2}\right) C_{k}^{3 / 2}(2 x-1) .
\end{gathered}
$$


The coefficients $B_{n}^{L O}\left(\mu_{F}^{2}\right)$ and $B_{k}^{N L O}\left(\mu_{F}^{2}\right)$ depend on the nonperturbative input $B_{n}$, as well as on the scales $\mu_{0}^{2}$ and $\mu_{F}^{2}$. Their exact form can be read from the results obtained from 23] and listed in $[8]^{1}$.

\section{CALCULATIONAL PROCEDURE}

Before proceeding with the calculation, we would like to point out some subtleties connected with the calculational procedure that (we think) deserve more explanation.

The hard-scattering amplitude $T_{H}$ is obtained by evaluating the $\gamma^{*}+\gamma \rightarrow q \bar{q}$ amplitude, which contains collinear singularities, owing to the fact that final state quarks are taken to be massless and on-shell. Since, by definition, $T_{H}$ is a finite quantity free of collinear singularities, these singularities should be subtracted. The $\gamma^{*}+\gamma \rightarrow q \bar{q}$ amplitude with the Lorentz structure factored out as in Eq. (2.1) and denoted by $T$ factorizes as

$$
T\left(u, Q^{2}\right)=T_{H}\left(x, Q^{2}, \mu_{F}^{2}\right) \otimes Z_{T, \text { col }}\left(x, u ; \mu_{F}^{2}\right),
$$

where, as usual, $u$ and $1-u$ denote the quark/antiquark longitudinal momentum fractions, $\mu_{F}^{2}$ is a factorization scale at which the separation of collinear singularities takes place and all collinear singularities are factorized in $Z_{T, c o l}$.

On the other hand, a process-independent distribution amplitude for a pion in a frame where $P^{+}=P^{0}+P^{3}=1$, $P^{-}=P^{0}-P^{3}=0$, and $P_{\perp}=0$ is defined [2, 20, 24] as

$$
\begin{aligned}
\Phi(u)= & \int \frac{d z^{-}}{2 \pi} e^{i(u-(1-u)) z^{-} / 2} \\
& \times\left\langle 0\left|\bar{\Psi}(-z) \frac{\gamma^{+} \gamma_{5}}{2 \sqrt{2}} \Omega \Psi(z)\right| \pi\right\rangle_{\left(z^{+}=z_{\perp}=0\right)},
\end{aligned}
$$

where

$$
\Omega=\exp \left\{i g \int_{-1}^{1} d s A^{+}(z s) z^{-} / 2\right\}
$$

is a path-ordered factor making $\Phi$ gauge invariant. The matrix element in (3.2) contains an ultraviolet divergence coming from the light-cone singularity at $z^{2}=0$ 2, 24. This divergence should be regulated, and after renormalization, which introduces a renormalization scale $\tilde{\mu}_{R}^{2}, z^{2}$ is effectively smeared over a region of order $z^{2}=-z_{\perp}^{2} \sim 1 / \tilde{\mu}_{R}^{2}$. As a result, the pion distribution amplitude $\Phi\left(v, \widetilde{\mu}_{R}^{2}\right)$ is obtained corresponding to the pion wave function integrated over the pion intrinsic

\footnotetext{
${ }^{1}$ It should, however, be pointed out that, in contrast to the expansion parameter $\alpha_{S}\left(\mu_{F}^{2}\right) /(4 \pi)$ from 2.11$)$, the expansion parameter $\alpha_{S}\left(\mu_{F}^{2}\right) / \pi$ was chosen in [8]. Hence, the expressions for $B_{n}^{L O(N L O)}$ from 8 should be modified accordingly.
}

transverse momentum up to the scale $\tilde{\mu}_{R}^{2}$. The distribution amplitude $\Phi\left(v, \tilde{\mu}_{R}^{2}\right)$ is a finite quantity and enters the convolution expression (2.2).

The unrenormalized pion distribution amplitude $\Phi(u)$ given in (3.2) and the distribution amplitude $\Phi\left(v, \tilde{\mu}_{R}^{2}\right)$ renormalized at the scale $\tilde{\mu}_{R}^{2}$ are (owning to the multiplicative renormalizability of the composite operator $\left.\bar{\Psi} \gamma^{+} \gamma_{5} \Omega \Psi\right)$ related by a multiplicative renormalizability equation

$$
\Phi(u)=Z_{\phi, r e n}\left(u, v ; \tilde{\mu}_{R}^{2}\right) \otimes \Phi\left(v, \tilde{\mu}_{R}^{2}\right) .
$$

By differentiating this equation with respect to $\tilde{\mu}_{R}^{2}$ one obtains the evolution equation (2.5), with the evolution potential $V$ given by

$$
V=-Z_{\phi, r e n}^{-1}\left(\tilde{\mu}_{R}^{2} \frac{\partial}{\partial \tilde{\mu}_{R}^{2}} Z_{\phi, r e n}\right) .
$$

For notational simplicity, here and where appropriate, we use the notation in which the convolution $(\otimes)$ is replaced by the matrix multiplication in $\mathrm{x}-\mathrm{y}$ space (unit matrix is given by $\mathbb{1} \equiv \delta(x-y))$.

The pion distribution amplitude as given in (3.2), with $|\pi\rangle$ being the physical pion state, of course, cannot be determined using perturbation theory. If the meson state $|\pi\rangle$ is replaced by a $|q \bar{q} ; t\rangle$ state composed of a free (collinear, massless, and on-shell) quark and antiquark (carrying momenta $t P$ and $(1-t) P$ ), the amplitude $(3.2)$ becomes

$$
\begin{aligned}
\tilde{\phi}(u, t)= & \int \frac{d z^{-}}{2 \pi} e^{i(u-(1-u)) z^{-} / 2} \\
& \times\left\langle 0\left|\bar{\Psi}(-z) \frac{\gamma^{+} \gamma_{5}}{2 \sqrt{2}} \Omega \Psi(z)\right| q \bar{q} ; t\right\rangle \frac{1}{\sqrt{N_{c}}} .
\end{aligned}
$$

Taking (3.6) into account, Eq. 3.2 can be written in the form

$$
\Phi(u)=\tilde{\phi}(u, t) \otimes\langle q \bar{q} ; t \mid \pi\rangle \sqrt{N_{c}} .
$$

The amplitude (3.6) can be treated perturbatively, making it possible to investigate the high-energy tail of the pion DA, to obtain $Z_{\phi, r e n}$ and to determine the DA evolution.

The distribution $\tilde{\phi}(u, t)$ is multiplicatively renormalizable and the UV singularities that are not removed by the renormalization of the fields and by the coupling constant renormalization, factorize in the renormalization constant $Z_{\phi, \text { ren }}$ at the renormalization scale $\tilde{\mu}_{R}^{2}$. Apart from UV singularities, the matrix element in (3.6) contains collinear singularities (since the initial state quarks are, as before, taken to be massless and on-shell), which are absorbed in $Z_{\phi, c o l}$ at the factorization scale $\mu_{0}^{2}$. Hence, one obtains

$$
\begin{aligned}
& \tilde{\phi}(u, t) \\
& \quad=Z_{\phi, r e n}\left(u, v ; \tilde{\mu}_{R}^{2}\right) \otimes \phi_{V}\left(v, s ; \tilde{\mu}_{R}^{2}, \mu_{0}^{2}\right) \otimes Z_{\phi, c o l}\left(s, t ; \mu_{0}^{2}\right) .
\end{aligned}
$$




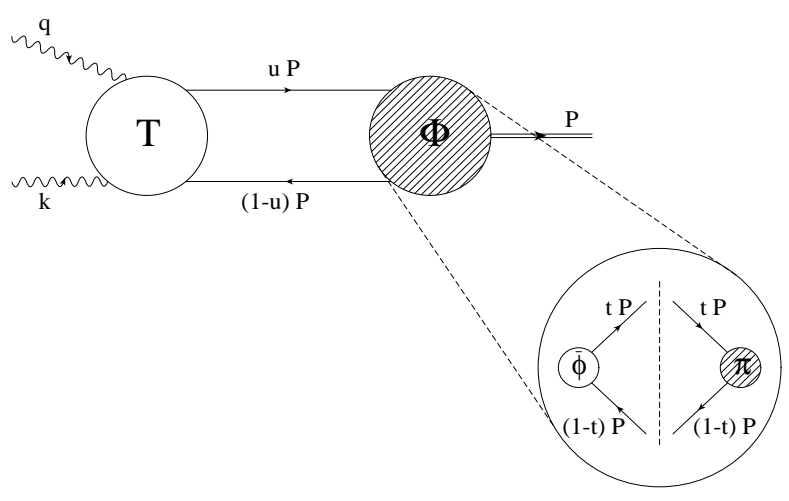

FIG. 2: Pictorial representation of the of the pion transition form factor calculational ingredients: $T$ represents the perturbatively calculable hard-scattering amplitude, while $\Phi$ is the pion distribution amplitude given by (3.2) which can be expressed, as in (3.7), in terms of the perturbatively calculable part $\tilde{\phi} 3.6$ and the perturbatively uncalculable part.

Upon combining Eqs. (3.7) and (3.8), the distribution $\Phi(u)$ can be written in the form

$$
\Phi(u)=Z_{\phi, r e n}\left(u, v ; \tilde{\mu}_{R}^{2}\right) \otimes \phi_{V}\left(v, s ; \tilde{\mu}_{R}^{2}, \mu_{0}^{2}\right) \otimes \Phi\left(s, \mu_{0}^{2}\right) .
$$

Here,

$$
\Phi\left(s, \mu_{0}^{2}\right)=Z_{\phi, c o l}\left(s, t ; \mu_{0}^{2}\right) \otimes\langle q \bar{q} ; t \mid \pi\rangle \sqrt{N_{c}}
$$

represents the nonperturbative input (containing all effects of collinear singularities, confinement, and pion bound-state dynamics) determined at the scale $\mu_{0}^{2}$, while $\phi_{V}\left(v, s ; \tilde{\mu}_{R}^{2}, \mu_{0}^{2}\right)$ governs the evolution of distribution amplitude to the scale $\tilde{\mu}_{R}^{2}$ :

$$
\Phi\left(v, \tilde{\mu}_{R}^{2}\right)=\phi_{V}\left(v, s ; \tilde{\mu}_{R}^{2}, \mu_{0}^{2}\right) \otimes \Phi\left(s, \mu_{0}^{2}\right),
$$

and satisfies the evolution equation

$$
\begin{aligned}
& \tilde{\mu}_{R}^{2} \frac{\partial}{\partial \tilde{\mu}_{R}^{2}} \phi_{V}\left(v, s, \tilde{\mu}_{R}^{2}, \mu_{0}^{2}\right) \\
& \quad=V\left(v, s^{\prime}, \tilde{\mu}_{R}^{2}\right) \otimes \phi_{V}\left(s^{\prime}, s, \tilde{\mu}_{R}^{2}, \mu_{0}^{2}\right) .
\end{aligned}
$$

By convoluting the "unrenormalized" (in the sense of collinear singularities) hard-scattering amplitude $T\left(u, Q^{2}\right)$ with the unrenormalized pion distribution amplitude $\Phi(u)$, given by Eqs. (3.1) and (3.4), respectively, one obtains (in a way analogous to [2, 25) the following expression for the pion transition form factor $F_{\gamma^{*} \gamma \pi}\left(Q^{2}\right)$ :

$$
F_{\gamma^{*} \gamma \pi}\left(Q^{2}\right)=\Phi^{\dagger}(u) \otimes T\left(u, Q^{2}\right) .
$$

The divergences of $T\left(u, Q^{2}\right)$ and $\Phi(u)$ cancel for $\tilde{\mu}_{R}^{2}=\mu_{F}^{2}$

$$
Z_{T, \text { col }}\left(x, u ; \mu_{F}^{2}\right) \otimes Z_{\phi, r e n}\left(u, v ; \mu_{F}^{2}\right)=\delta(x-v),
$$

and the usual expression (2.2) emerges, where the pion transition form factor is expressed in a form of the convolution of two finite amplitudes:

$$
F_{\gamma \pi}\left(Q^{2}\right)=T_{H}\left(x, Q^{2}, \mu_{F}^{2}\right) \otimes \Phi^{*}\left(x, \mu_{F}^{2}\right) .
$$

It is worth pointing out that the scale $\mu_{F}^{2}$ representing the boundary between the low- and high-energy parts in (2.2) is, at same time, the separation scale for collinear singularities in $T\left(u, Q^{2}\right)$, on the one hand, and the renormalization scale for UV singularities appearing in the perturbatively calculable part of the distribution amplitude $\Phi(u)$, on the other hand. The calculational procedure explained above is illustrated in Fig. 2.

Our main goal in this work is to determine the BLM scale for the pion transition form factor. To achieve that, we make use of the calculational procedure outlined above and in the following sections calculate the LO, NLO, and $n_{f}$-proportional NNLO contributions to the perturbative expansions of both the hard-scattering amplitude and the distribution amplitude.

This is the first calculation of the hard-scattering amplitude $T\left(u, Q^{2}\right)$ of an exclusive process with the NNLO terms taken into account. The subtraction (separation) of collinear divergences at the NNLO is significantly more demanding than that at the NLO. Owing to the fact that the process under consideration contains one pseudoscalar meson, the calculation is further complicated by the $\gamma_{5}$ ambiguity related to the use of the dimensional regularization method to treat divergences.

In order to correctly subtract the collinear divergences and determine the right treatment of the $\gamma_{5}$ matrix, we determine the LO, NLO, and $n_{f}$-proportional NNLO contributions to the distribution $\tilde{\phi}(u, t)$ given in (3.6), and by that, following (3.7 3.9), the renormalization constant $Z_{\phi, r e n}$ and the evolutional part $\phi_{V}$ of the distribution amplitude $\Phi(u)$ (3.2). Since, there is no $\gamma_{5}$ ambiguity in the DA calculation, the $\gamma_{5}$ ambiguity present in the hard-scattering calculation is resolved using (3.14). As an additional check, we employ two $\gamma_{5}$ schemes in our calculation. Finally, we obtain the ( $\gamma_{5}$ scheme independent) prediction for the pion transition form factor $F_{\gamma^{*} \gamma \pi}$ up to the $n_{f}$-proportional NNLO terms, expressed in terms of the finite quantities $T_{H}\left(x, Q^{2}, \mu_{F}^{2}\right)$ and $\Phi\left(x, \mu_{F}^{2}\right)$.

\section{LO, NLO, AND $n_{f}$-PROPORTIONAL NNLO UNRENORMALIZED CONTRIBUTIONS TO THE HARD-SCATTERING AND THE DISTRIBUTION AMPLITUDES}

In this section we present the calculation of the LO, $\mathrm{NLO}$, and $n_{f}$-proportional NNLO contributions to the hard-scattering amplitude and the perturbatively calculable part of the distribution amplitude.

\section{A. Contributions to the hard-scattering amplitude}

The hard-scattering amplitude $T_{H}$ for the pion transition form factor is obtained by evaluating the $\gamma^{*} \gamma \rightarrow q \bar{q}$ amplitude for the parton subprocess, which is described by the Feynman diagrams of Fig. 1.

The $q \bar{q}$ pair has to be projected into a negative-parity 

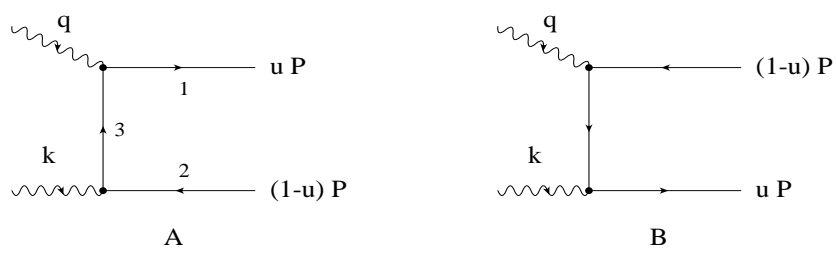

FIG. 3: Lowest-order Feynman diagrams contributing to the $\gamma^{*} \gamma \rightarrow q \bar{q}$ amplitude.

and spin 0 (pseudoscalar) state. This is achieved by introducing the projection operator $\gamma_{5} P / \sqrt{2}$ and taking the trace over a fermion loop. On the other hand, the color-singlet nature of the $q \bar{q}$ state is taken into account by introducing the factor $\sum_{\alpha=1}^{3} \delta_{\alpha \beta} / \sqrt{N_{c}}$, and taking the trace over the color indices. Also, the flavor function $(u \bar{u}-d \bar{d}) / \sqrt{2}$ should be included.

The hard-scattering amplitude $T\left(u, Q^{2}\right)$ can generally be expressed as an expansion in $\alpha_{S}$

$$
\begin{aligned}
T\left(u, Q^{2}\right)= & \frac{N_{T}}{Q^{2}}\left\{T^{(0)}(u)+\frac{\alpha_{S}}{4 \pi} T^{(1)}(u)\right. \\
& +\frac{\alpha_{S}^{2}}{(4 \pi)^{2}}\left[\left(-\frac{2}{3} n_{f}\right) T^{\left(2, n_{f}\right)}(u)+\cdots\right] \\
& +\cdots\} .
\end{aligned}
$$

\section{LO contributions}

In the LO approximation there are only two Feynman diagrams that contribute to the $\gamma^{*} \gamma \rightarrow q \bar{q}$ amplitude. They are shown in Fig. 3 . The contribution of diagram $A$ (after $i e^{2} \epsilon^{\mu \nu \alpha \beta} P_{\alpha} q_{\beta}$ is factored out) is given by

$$
T_{A}=\frac{N_{T}}{Q^{2}} \frac{1}{1-u},
$$

where

$$
N_{T}=2 \sqrt{2 N_{c}} C_{\pi}
$$

and

$$
C_{\pi}=\frac{e_{u}^{2}-e_{d}^{2}}{\sqrt{2}}=\frac{\sqrt{2}}{6}
$$

is the factor taking into account the flavor content of the $q \bar{q}$ pair. The contribution of diagram $B$ is obtained by making the replacement $u \rightarrow(1-u)$ in (4.2). Therefore, the lowest-order (QED) contribution to the $\gamma^{*} \gamma \rightarrow q \bar{q}$ amplitude, i.e., to $T\left(u, Q^{2}\right)$ given in (4.1), is

$$
T^{(0)}(u)=\frac{1}{1-u}+(u \rightarrow 1-u)
$$
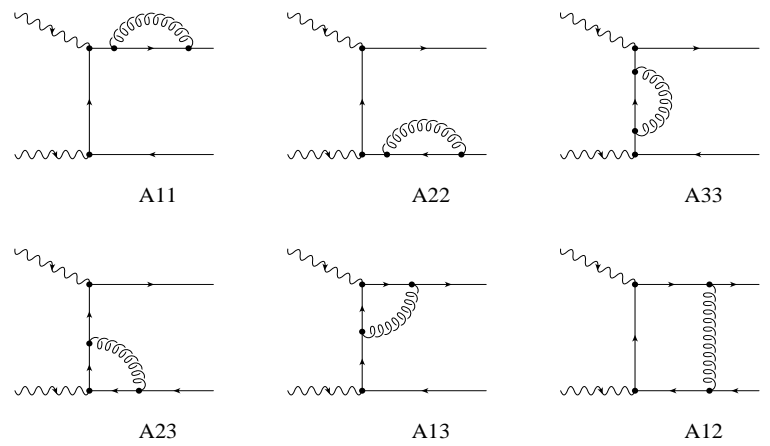

FIG. 4: Distinct one-loop Feynman diagrams contributing to the $\gamma^{*} \gamma \rightarrow q \bar{q}$ amplitude, generated from diagram $A$ of Fig. 3 by inserting a gluon line.

\section{NLO contributions}

At NLO there are 12 one-loop Feynman diagrams contributing to the $\gamma^{*} \gamma \rightarrow q \bar{q}$ amplitude. They can be generated by inserting an internal gluon line into the lowestorder diagrams of Fig. 3. We use the notation where $A i j$ is the diagram obtained from diagram $A$ by inserting the gluon line connecting the lines $i$ and $j$, where $i, j=1,2,3$. Since NLO, and all higher-order, diagrams generated from diagram $B$ can be obtained from the corresponding diagrams generated from diagram $A$ by using the substitution $u \rightarrow(1-u)$, the total number of NLO diagrams to be evaluated is 6 . They are shown in Fig. 4 .

These diagrams contain ultraviolet (UV) singularities, and owing to the fact that the final state quarks are massless and on shell they also contain collinear singularities. To regularize these singularities, we use dimensional regularization in $D=4-2 \epsilon$ space-time dimensions.

As is well known, dimensional regularization leads to an ambiguity when dealing with the pseudoscalar matrix $\gamma_{5}$. The reason for this lies in the fact that the matrix $\gamma_{5}$ cannot be unambiguously defined in $D \neq 4$ dimensions. In practice, the difficulty arises in evaluating a trace containing a single $\gamma_{5}$. We address this problem in detail in Appendix A.

In order to make sure that our results for the pion transition form factor are $\gamma_{5}$ scheme independent, we have evaluated all the contributions using two schemes: the naive- $\gamma_{5}$ scheme [16] and the 't Hooft-Veltman (HV) scheme 117, 18], defined by (A1) and (A3), respectively.

A few remarks concerning the diagrams with quark self-energy corrections where the quark momentum $p$ is on-shell $(A 11, A 22)$ are in order. Since these corrections modify external legs, each of these diagrams is accompanied with a factor of $1 / 2$ coming from the expansion of the quark field renormalization constant $\sqrt{Z_{2}}$. In dimensional regularization, the contributions of each of these diagrams turn out to be proportional to $\left(p^{2}\right)^{-\epsilon}$ and, therefore, vanish when $p^{2}=0$. On closer inspection, 
TABLE I: Contributions $\widehat{T}_{A i j}^{U V}$ and $\widehat{T}_{A i j}^{I R}$ (defined in (4.6)) of Feynman diagrams shown in Fig. 1 . Apart from the result denoted by $12_{\delta=1}$, the listed results correspond to the results obtained using the HV scheme, or equivalently, to the results obtained in the naive- $\gamma_{5}$ scheme with $\gamma_{5}$ being outside the contracting $\gamma$ matrices. The result denoted by $12_{\delta=1}$ has been obtained using the naive- $\gamma_{5}$ scheme with $\gamma_{5}$ placed between the contracting $\gamma$ matrices and between two Dirac slashed loop momenta.

\begin{tabular}{ccc}
\hline \hline$i j$ & \multicolumn{1}{c}{$\widehat{T}_{A i j}^{U V}$} & $\widehat{T}_{A i j}^{I R}$ \\
\hline $11(22)$ & $-\frac{1}{2}(1-\epsilon)\left(1-\frac{\epsilon}{2}\right)(1-u)^{-\epsilon}$ & $-\frac{1}{2}(1-\epsilon)\left(1-\frac{\epsilon}{2}\right)(1-u)^{-\epsilon}$ \\
33 & $(1-\epsilon)(1-u)^{-\epsilon}$ & $(2+\epsilon)(1-u)^{-\epsilon}$ \\
23 & $(1-\epsilon)\left[(1-u)^{-\epsilon}+\frac{1}{u}\left(1-(1-u)^{-\epsilon}\right)\right]$ & {$\left[(2+\epsilon)(1-u)^{-\epsilon}+\left(\frac{2}{\epsilon}+\epsilon\right) \frac{1}{u}\left(1-(1-u)^{-\epsilon}\right)\right]$} \\
13 & 0 & $-2(1+\epsilon)\left(\frac{1}{\epsilon}+2\right) \frac{1-u}{u}\left(1-(1-u)^{-\epsilon}\right)$ \\
$12_{\delta=0}$ & 0 & $-2(1-\epsilon) \frac{1}{\epsilon} \frac{1-u}{u}\left(1-(1-u)^{-\epsilon}\right)$
\end{tabular}

however, one finds that this vanishing is a result of the cancellation of a UV pole with collinear pole. The UV pole contributes to the renormalization of the quark fields (already taken into account by the factor $\sqrt{Z_{2}}$ ) and eventually leads to a correct running of the coupling constant.

The contribution of any of the diagrams Aij shown in Fig. 4 can be generally expressed as

$$
T_{A i j}=\frac{N_{T}}{Q^{2}} \frac{1}{1-u} \frac{\alpha_{S}}{4 \pi} C_{F} \widetilde{T}_{A i j},
$$

where $C_{F}=4 / 3$ is the color factor (the same for all diagrams), while $\widetilde{T}_{A i j}$ is defined by

$$
\begin{aligned}
\widetilde{T}_{A i j}= & \left(\Gamma_{U V}^{(0)}(\epsilon) \frac{1}{1-2 \epsilon} \widehat{T}_{A i j}^{U V}\right. \\
& \left.+\Gamma_{I R}^{(0)}(\epsilon) \frac{1}{1-2 \epsilon} \widehat{T}_{A i j}^{I R}\right)\left(\frac{\mu^{2}}{Q^{2}}\right)^{\epsilon}
\end{aligned}
$$

with the following abbreviations

$$
\begin{aligned}
\Gamma_{U V}^{(0)}(\epsilon) & =\Gamma(\epsilon) \frac{\Gamma(1-\epsilon) \Gamma(1-\epsilon)}{\Gamma(1-2 \epsilon)}(4 \pi)^{\epsilon} \\
\Gamma_{I R}^{(0)}(\epsilon) & =\Gamma(1+\epsilon) \frac{\Gamma(-\epsilon) \Gamma(1-\epsilon)}{\Gamma(1-2 \epsilon)}(4 \pi)^{\epsilon} .
\end{aligned}
$$

The first $\Gamma$ function on the right-hand side of Eqs. (4.7) originates from the loop momentum integration, while the integration over Feynman parameters produces $\Gamma$ s collected in a fraction. Consequently, the singularity contained in $\Gamma(\epsilon)$ appearing in (4.7a) is of UV origin, while the singularity contained in $\Gamma(-\epsilon)$ appearing in $(4.7 \mathrm{~b})$ is of infrared (IR) origin. It should be pointed out, however, that none of the diagrams of Fig. 1 1 contains a soft (genuine IR) singularity, so that the, here and in the following, subscript (and / or the superscript) IR signifies the collinear singularity. If the relation

$$
\Gamma(z) \Gamma(1-z)=\frac{\pi}{\sin \pi z}
$$

is taken into account in (4.7), one finds that

$$
\Gamma_{U V}^{(0)}(\epsilon)=-\Gamma_{I R}^{(0)}(\epsilon)
$$

Nevertheless, we continue to keep track of the origin of the UV and collinear singularities.

The contributions $\widehat{T}_{A i j}^{U V}$ and $\widehat{T}_{A i j}^{I R}$ of the individual diagrams are given in Table If. Following the explanations and notation given in App. A, we list the contributions obtained using the HV scheme, which are equivalent to the results obtained in the naive- $\gamma_{5}$ scheme with $\gamma_{5}$ being positioned outside the contracted $\gamma$ matrices. For diagram $A 12$ we also list the contribution obtained in the naive- $\gamma_{5}$ scheme corresponding to the case where $\gamma$ matrices are contracted through the string of $\gamma$ matrices of the form $\not \gamma_{5} \not l$. As elaborated in Appendix A, the $\gamma_{5}$ ambiguity in diagrams $A 11, A 22, A 33, A 23$, and $A 13$ has been resolved with the help of QED Ward identities. The remaining ambiguity in diagram $A 12$ is parameterized by $\delta$, taking the value 0 for the first choice for handling $\gamma_{5}$ in diagram $A 12$, and 1 for the second. Our results listed in Table [ are in agreement with [10] (but see the comments in App. A).

The NLO contribution $T^{(1)}(u)$ from Eq. (4.1) is of the form

$$
\begin{aligned}
T^{(1)}(u)= & {\left[\Gamma_{U V}^{(0)}(\epsilon) \frac{1}{1-2 \epsilon} \bar{T}_{U V}^{(1)}(u)\right.} \\
& \left.+\Gamma_{I R}^{(0)}(\epsilon) \frac{1}{1-2 \epsilon} \bar{T}_{I R}^{(1)}(u)\right]\left(\frac{\mu^{2}}{Q^{2}}\right)^{\epsilon}
\end{aligned}
$$



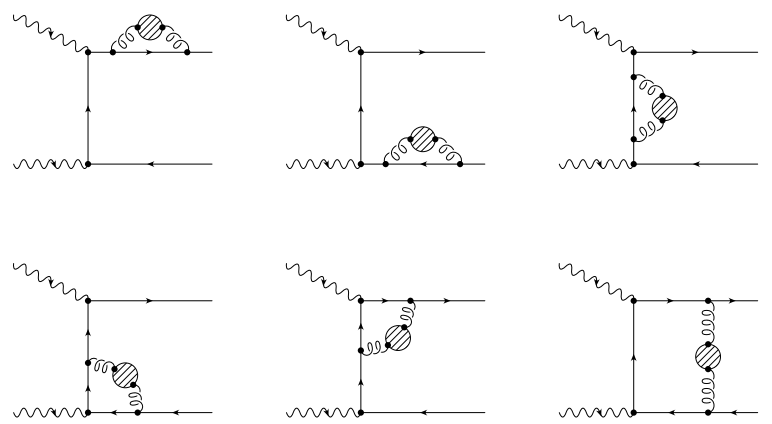

FIG. 5: Distinct vacuum-polarization two-loop Feynman diagrams contributing to the $\gamma^{*} \gamma \rightarrow q \bar{q}$ amplitude, which have been obtained from the one-loop diagrams of Fig. 4 by inserting the vacuum-polarization bubbles.

where

$$
\begin{aligned}
& \bar{T}_{U V}^{(1)}(u) \\
& =C_{F} \frac{1}{1-u}(1-\epsilon)\left[\frac{\epsilon}{2}(1-u)^{-\epsilon}+\frac{1}{u}\left(1-(1-u)^{-\epsilon}\right)\right] \\
& \quad+(u \rightarrow 1-u),
\end{aligned}
$$

and

$$
\begin{aligned}
& \bar{T}_{I R}^{(1)}(u) \\
& =C_{F} \frac{1}{1-u}\left[4+2 \epsilon-\left(1-\frac{3}{2} \epsilon+\frac{1}{2} \epsilon^{2}\right)(1-u)^{-\epsilon}\right. \\
& \quad+\left(\frac{2}{\epsilon}-4-\epsilon+(4 \delta-3) \frac{1-u}{u}(2+\epsilon)\right) \\
& \left.\quad \times\left(1-(1-u)^{-\epsilon}\right)\right]+(u \rightarrow 1-u)
\end{aligned}
$$

\section{3. $n_{f}$-proportional NNLO contributions}

By inserting the vacuum polarization bubbles in the NLO diagrams of Fig. 4 , the NNLO diagrams displayed in Fig. 5 are obtained. The vacuum polarization insertion is (in the Feynman gauge) given by the replacement

$$
-i g^{\kappa \lambda} \frac{\delta_{a b}}{l^{2}+i \eta} \rightarrow-i\left(g^{\kappa \lambda}-\frac{l^{\kappa} l^{\lambda}}{l^{2}+i \eta}\right) \frac{\delta_{a b}}{l^{2}+i \eta} \Pi\left(l^{2}\right),
$$

where

$$
\begin{aligned}
\Pi\left(l^{2}\right)= & \frac{g^{2}}{(4 \pi)^{2}}\left(\frac{\mu^{2}}{-l^{2}-i \eta}\right)^{\epsilon}\left[(5-3 \epsilon)-\frac{2}{3}(1-\epsilon) n_{f}\right] \\
& \times \frac{1}{\left(1-\frac{2}{3} \epsilon\right)(1-2 \epsilon)} \Gamma(\epsilon) \frac{\Gamma(1-\epsilon) \Gamma(1-\epsilon)}{\Gamma(1-2 \epsilon)}(4 \pi)^{\epsilon},
\end{aligned}
$$

and, due to gauge invariance of the complete finite order contribution (which we have used as an additional check of our calculation), it can effectively be described by

$$
-i g^{\kappa \lambda} \frac{\delta_{a b}}{l^{2}+i \eta} \rightarrow-i g^{\kappa \lambda} \frac{\delta_{a b}}{l^{2}+i \eta} \Pi\left(l^{2}\right) \text {. }
$$

We are interested only in $n_{f}$-proportional part (from the quark loops inserted in the gluon propagator)

$$
\Pi_{n_{f}}\left(l^{2}\right)=\left(-\frac{2}{3} n_{f}\right) \frac{1}{\left(l^{2}+i \eta\right)^{\epsilon}} \frac{g^{2}}{(4 \pi)^{2}} f_{n_{f}}\left(\epsilon, \mu^{2}\right),
$$

where $f_{n_{f}}$ is defined by

$$
f_{n_{f}}\left(\epsilon, \mu^{2}\right)=\left(\frac{\mu^{2}}{-1-i \eta^{\prime}}\right)^{\epsilon} \frac{1-\epsilon}{\left(1-\frac{2}{3} \epsilon\right)(1-2 \epsilon)} \Gamma_{U V}^{(0)}(\epsilon)
$$

The contributions of the two-loop Feynman diagrams shown in Fig. 5 can than be generally written as

$$
T_{(A i j)_{n f}}=\frac{N_{T}}{Q^{2}} \frac{1}{1-u} \frac{\alpha_{S}^{2}}{(4 \pi)^{2}} C_{F}\left(-\frac{2}{3} n_{f}\right) \widetilde{T}_{(A i j)_{n f}},
$$

where

$$
\begin{aligned}
& \widetilde{T}_{(A i j)_{n f}} \\
& =\left(\Gamma_{U V}^{(0)}(\epsilon) \Gamma_{U V}^{(1)}(\epsilon) \frac{(1-\epsilon)}{\left(1-\frac{2}{3} \epsilon\right)(1-2 \epsilon)(1-3 \epsilon)} \widehat{T}_{(A i j)_{n f}}^{U V}\right. \\
& \left.\quad+\Gamma_{U V}^{(0)}(\epsilon) \Gamma_{I R}^{(1)}(\epsilon) \frac{(1-\epsilon)}{\left(1-\frac{2}{3} \epsilon\right)(1-2 \epsilon)(1-3 \epsilon)} \widehat{T}_{(A i j)_{n f}}^{I R}\right) \\
& \quad \times\left(\frac{\mu^{2}}{Q^{2}}\right)^{2 \epsilon},
\end{aligned}
$$

while, similarly to 4.7 , the abbreviations

$$
\begin{aligned}
\Gamma_{U V}^{(1)}(\epsilon) & =\frac{\Gamma(2 \epsilon)}{\Gamma(1+\epsilon)} \frac{\Gamma(1-2 \epsilon) \Gamma(1-\epsilon)}{\Gamma(1-3 \epsilon)}(4 \pi)^{\epsilon} \\
\Gamma_{I R}^{(1)}(\epsilon) & =\frac{\Gamma(1+2 \epsilon)}{\Gamma(1+\epsilon)} \frac{\Gamma(-2 \epsilon) \Gamma(1-\epsilon)}{\Gamma(1-3 \epsilon)}(4 \pi)^{\epsilon}
\end{aligned}
$$

have been introduced.

The contributions $\widehat{T}_{(A i j)_{n f}}^{U V, I R}$ of the individual diagrams are listed in Table III. The integrals $I_{i}(u, \epsilon)(i=1,2,3)$ appearing in this table are defined as

$$
\begin{aligned}
& I_{1}(u, \epsilon) \equiv I(u ; \epsilon, 2 \epsilon) \\
& I_{2}(u, \epsilon) \equiv I(u ; \epsilon, 1+2 \epsilon) \\
& I_{3}(u, \epsilon) \equiv I(u ; 1+\epsilon, 1+2 \epsilon)
\end{aligned}
$$

where

$$
\begin{aligned}
I(u ; a, c) & =\int_{0}^{1} d y \frac{y^{a}}{(1-u y)^{c}} \\
& =\frac{1}{1+a}{ }_{2} F_{1}(c, 1+a, 2+a ; u) .
\end{aligned}
$$

As far as the $\gamma_{5}$-scheme dependence of the NNLO diagram contributions is concerned, it is the same as for the NLO diagrams from Table $\mathbb{1}$. 


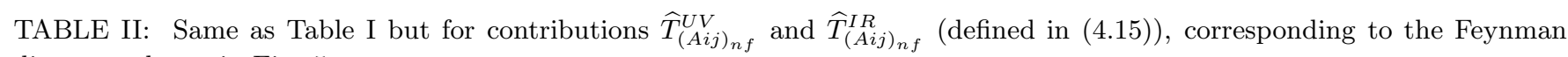
diagrams shown in Fig. 5 .

\begin{tabular}{ccc}
\hline \hline$i j$ & \multicolumn{1}{c}{$\widehat{T}_{(A i j)_{n}}^{U V}$} & $\widehat{T}_{(A i j)_{n} f}^{I R}$ \\
\hline $11(22)$ & $-\frac{1}{2} \frac{(1-\epsilon)^{2}\left(1-\frac{\epsilon}{2}\right)}{\left(1+\frac{\epsilon}{2}\right)\left(1-\frac{3}{2} \epsilon\right)}(1-u)^{-2 \epsilon}$ & $-\frac{1}{2} \frac{(1-\epsilon)^{2}\left(1-\frac{\epsilon}{2}\right)}{\left(1+\frac{\epsilon}{2}\right)\left(1-\frac{3}{2} \epsilon\right)}(1-u)^{-2 \epsilon}$ \\
33 & $-\frac{(1-\epsilon)^{2}}{\left(1-\frac{3}{2} \epsilon\right)}(1-u)^{-2 \epsilon}$ & 0 \\
23 & $\frac{(1-\epsilon)^{2}}{\left(1-\frac{3}{2} \epsilon\right)}(1-u)^{-2 \epsilon}$ & $\frac{\left(2-\epsilon-2 \epsilon^{2}\right)}{\left(1-\frac{3}{2} \epsilon\right)}(1-u)^{-2 \epsilon}$ \\
13 & $\frac{(1-\epsilon)^{3}}{\left(1-\frac{3}{2} \epsilon\right)} I_{1}(u, \epsilon)$ & $\frac{(1-\epsilon)}{\left(1-\frac{3}{2} \epsilon\right)}\left[2 \epsilon(1-\epsilon) I_{2}(u, \epsilon)-\left(2-\epsilon-2 \epsilon^{2}\right) u I_{3}(u, \epsilon)\right]$ \\
$12_{\delta=0}$ & 0 & $-2(1+\epsilon)\left[-(1-u)^{-2 \epsilon}-2 \epsilon I_{2}(u, \epsilon)-(1-\epsilon) u I_{3}(u, \epsilon)\right](1-u)$ \\
$12_{\delta=1}$ & 0 & $-2(1-\epsilon)\left[-(1-u)^{-2 \epsilon}-(1-\epsilon) u I_{3}(u, \epsilon)\right](1-u)$ \\
\hline \hline
\end{tabular}

The $n_{f}$-proportional NNLO contribution $T_{n_{f}}^{(2)}(u)$ from Eq. (4.1) takes the form

$$
\begin{aligned}
T^{\left(2, n_{f}\right)}(u)= & {\left[\Gamma_{U V}^{(0)}(\epsilon) \Gamma_{U V}^{(1)}(\epsilon) \frac{(1-\epsilon)}{\left(1-\frac{2}{3} \epsilon\right)(1-2 \epsilon)(1-3 \epsilon)} \bar{T}_{U V}^{\left(2, n_{f}\right)}(u)\right.} \\
& \left.+\Gamma_{U V}^{(0)}(\epsilon) \Gamma_{I R}^{(1)}(\epsilon) \frac{(1-\epsilon)}{\left(1-\frac{2}{3} \epsilon\right)(1-2 \epsilon)(1-3 \epsilon)} \bar{T}_{I R}^{\left(2, n_{f}\right)}(u)\right]\left(\frac{\mu^{2}}{Q^{2}}\right)^{2 \epsilon}
\end{aligned}
$$

where

$$
\begin{aligned}
\bar{T}_{U V}^{\left(2, n_{f}\right)}(u)= & C_{F} \frac{1}{1-u} \frac{(1-\epsilon)^{2}}{1-\frac{3}{2} \epsilon}\left[(1-\epsilon) I_{1}(u, \epsilon)-\frac{1-\frac{\epsilon}{2}}{1+\frac{\epsilon}{2}}(1-u)^{-2 \epsilon}\right]+(u \rightarrow 1-u), \\
\bar{T}_{I R}^{\left(2, n_{f}\right)}(u)= & C_{F} \frac{1}{1-u}\left\{\left[\frac{1+\frac{5}{2} \epsilon-\frac{9}{2} \epsilon^{2}-\frac{1}{2} \epsilon^{3}}{\left(1+\frac{\epsilon}{2}\right)\left(1-\frac{3}{2} \epsilon\right)}+2(1-(2 \delta-1) \epsilon)(1-u)\right](1-u)^{-2 \epsilon}\right. \\
& +2 \epsilon\left[\frac{(1-\epsilon)^{2}}{1-\frac{3}{2} \epsilon}-(2 \delta-2)(1+\epsilon)(1-u)\right] I_{2}(u, \epsilon) \\
& \left.-2\left[\frac{\left(1-\frac{\epsilon}{2}-\epsilon^{2}\right)(1-\epsilon)}{1-\frac{3}{2} \epsilon}-(1-(2 \delta-1) \epsilon)(1-\epsilon)(1-u)\right] u I_{3}(u, \epsilon)\right\} \\
& +(u \rightarrow 1-u) .
\end{aligned}
$$

Our results are expressed in a compact form in which the complete functional dependence on the dimensional parameter $\epsilon$ is retained. This is in contrast to the expansion over $\epsilon$ often encountered in the literature. In this expansion, nonleading terms in $\epsilon$ are neglected before renormalization and factorization of collinear singularities. As we show in Sec. V, it is advantageous (both for the simplicity and accuracy check of the calculation) not to expand the functions $\Gamma_{U V, I R}^{(0,1)}$ over $\epsilon$.

\section{B. Contributions to the perturbatively calculable part of the distribution amplitude}

In Sec. II we have defined the distribution amplitude $\tilde{\phi}(u, t)$ (3.6) representing the perturbatively calculable part of the pion distribution amplitude. Following [20], we have rederived the Feynman rules for this operator in the Feynman gauge. They are listed in Appendix B.

We now proceed to calculate LO, NLO, and $n_{f^{-}}$ 


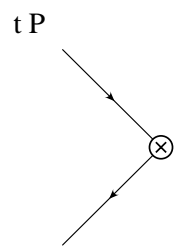

(1-t) P

FIG. 6: The LO diagram contributing to the $\tilde{\phi}(u, t)$ distribution (3.6), i.e., (B3).

proportional NNLO contributions to the $\tilde{\phi}(u, t)$ distribution amplitude defined in (3.6), or equivalently in (B3).

Contrary to [20, 24], we use dimensional regularization to regularize both UV and collinear singularities ${ }^{2}$. This enables us to combine these results with the hardscattering results, also obtained by employing the dimensional regularization. Compared to the hard-scattering amplitude calculation, calculation of the $\tilde{\phi}$ amplitude is complicated by the fact that noncovariant $l^{+}, l^{-}$and $\delta$-function terms (see (B4) and (B5)) appear in the loopmomenta. To deal with these types of terms and in order to simplify the expressions we follow the prescription given in 20 .

The presence of two $\gamma_{5}$ matrices in the traces has enabled their unambiguous treatment in the naive- $\gamma_{5}$ scheme (see Appendix A). Additionally, we have obtained the results using the HV scheme, which, however, introduces the "spurious" anomalous terms, and hence the additional renormalization is required. The corresponding renormalization constant will be determined by comparing the results obtained in the naive- $\gamma_{5}$ with those obtained in the HV scheme.

The perturbatively calculable $\tilde{\phi}(u, t)$ amplitude can be represented as a series in $\alpha_{S}$

$$
\begin{aligned}
\tilde{\phi}(u, t)= & \tilde{\phi}^{(0)}(u, t)+\frac{\alpha_{S}}{4 \pi} \tilde{\phi}^{(1)}(u, t) \\
& +\frac{\alpha_{S}^{2}}{(4 \pi)^{2}}\left[\left(-\frac{2}{3} n_{f}\right) \tilde{\phi}^{\left(2, n_{f}\right)}(u, t)+\cdots\right]+\cdots
\end{aligned}
$$

2 The evolutional behavior of the DA can be extracted from 3.6 even when using dimensional regularization for both UV and mass singularities. We introduce the auxiliary scale $\tilde{Q}^{2}$ and we insist on discriminating between UV and collinear singularities. Otherwise, the UV and collinear part of higher-order corrections would cancel, leading to $\tilde{\phi}(u, t)=\delta(u-t)$.

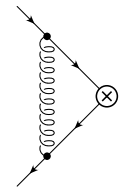

A

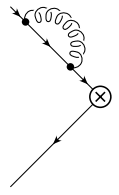

B1

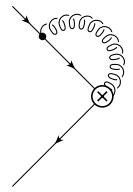

C1

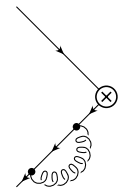

B2

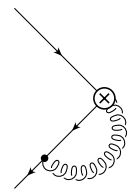

$\mathrm{C} 2$
FIG. 7: The one-loop diagrams contributing to the $\tilde{\phi}(u, t)$ distribution (3.6), i.e., (B3) (in the Feynman gauge).

\section{LO contribution}

The contribution of the LO Feynman diagram displayed in Fig. 6 reads

$$
\begin{aligned}
& \tilde{\phi}^{(0)}(u, t)=\delta(u-t) . \\
& \text { 2. NLO contributions }
\end{aligned}
$$

At NLO there are 5 one-loop Feynman diagrams contributing when the Feynman gauge is used. They are displayed in Fig. 7. The general form of these individual contributions (denoted by $X$ ) is given by

$$
\begin{aligned}
& \tilde{\phi}_{X}(u, t) \\
& =\frac{\alpha_{S}}{4 \pi} K_{X}(u, t)\left\{\frac{(4 \pi)^{2}}{i}\left[\mu^{2 \epsilon} \int \frac{d^{D} l}{(2 \pi)^{D}} \frac{1}{\left(l^{2}+i \eta\right)^{2}}\right]\right\},
\end{aligned}
$$

where

$$
K_{X}(u, t)=2 C_{F} \int_{0}^{1} d y F_{X}(u, t ; y)
$$

and the $F_{X}(u, t ; y)$ and $K_{X}(u, t)$ contributions can be read from Table III. The notation $u_{1}=u, u_{2}=1-u$ has been introduced, as well as the usual definition of the "+" form

$$
\{F(x, y)\}_{+} \equiv F(x, y)-\delta(x-y) \int_{0}^{1} d z F(z, y),
$$


TABLE III: Contributions $F_{X}$ appearing in Eqs. 4.25) and (4.31) and corresponding to the diagrams $(X)$ displayed in Fig. 月 and Fig. 8. We list also the general results for the integrals appearing in Eqs. (4.25) and (4.31), and parameterized by $\kappa=0$ and $\kappa=\epsilon$, respectively.

\begin{tabular}{ccc}
\hline \hline $\mathrm{X}$ & $F_{X}(u, t ; y)$ & {$\left[\int_{0}^{1} d y y^{\kappa} F_{X}(u, t ; y)\right]_{\kappa=0, \epsilon}$} \\
\hline$A$ & $(1-\epsilon)\left[u_{1} \delta\left(u_{1}-y t_{1}\right)+u_{2} \delta\left(u_{2}-y t_{2}\right)\right]$ & $(1-\epsilon)\left[\frac{u_{1}}{t_{1}} \theta\left(t_{1}-u_{1}\right)\left(\frac{u_{1}}{t_{1}}\right)^{\kappa}+\left(\begin{array}{c}u_{1} \rightarrow u_{2} \\
t_{1} \rightarrow t_{2}\end{array}\right)\right]$ \\
$A+B 1+B 2$ & $(1-\epsilon)\left\{u_{1} \delta\left(u_{1}-y t_{1}\right)+u_{2} \delta\left(u_{2}-y t_{2}\right)\right\}_{+}$ & $(1-\epsilon)\left\{\frac{u_{1}}{t_{1}} \theta\left(t_{1}-u_{1}\right)\left(\frac{u_{1}}{t_{1}}\right)^{\kappa}+\left(\begin{array}{c}u_{1} \rightarrow u_{2} \\
t_{1} \rightarrow t_{2}\end{array}\right)\right\}_{+}$ \\
$C 1$ & $-\left\{\frac{u_{1}}{u_{1}-t_{1}} \delta\left(u_{1}-y t_{1}\right)\right\}_{+}$ & \\
\hline \hline
\end{tabular}

the presence of which reflects the chiral symmetry conservation. This "+" form is a consequence of the fact that the axial current is conserved in the chiral limit, and represents a general all-order property [21].

By definition, the $D$ dimensional integral in Eq. (4.24) gives zero in dimensional regularization, but only if we do not distinguish between UV and collinear singularities ${ }^{3}$. By discriminating between the singularities of different

3 The $D$ dimensional integrals appearing in Eqs. 4.24 and (4.30 are of the form

$$
I_{[\alpha]}=\mu^{2 \epsilon} \int \frac{d^{D} l}{(2 \pi)^{D}} \frac{1}{\left(l^{2}+i \eta\right)^{\alpha}} .
$$

By employing

$$
I_{[\alpha]}=\left[\mu^{2 \epsilon} \int \frac{d^{D} l}{(2 \pi)^{D}} \frac{(l-p)^{2}}{\left(l^{2}+i \eta\right)^{\alpha}\left((l-p)^{2}+i \eta\right)}\right]_{p^{2} \neq 0},
$$

and insisting on distinguishing the $\Gamma$ functions obtained from the loop-momentum integrations and $\Gamma$ functions from the Feynman parameter integration, it can be shown that

$$
\begin{aligned}
I_{[\alpha]}= & \frac{i}{(4 \pi)^{2}} \frac{1}{\left(p^{2}+i \eta\right)^{\alpha-2}}\left(\frac{4 \pi \mu^{2}}{-p^{2}-i \eta}\right)^{\epsilon} \\
\times & {\left[\frac{\Gamma(\alpha-2+\epsilon)}{\Gamma(\alpha)} \frac{\Gamma(3-\alpha-\epsilon) \Gamma(2-\epsilon)}{\Gamma(5-\alpha-2 \epsilon)}(2-\epsilon)\right.} \\
& \left.+\frac{\Gamma(\alpha-1+\epsilon)}{\Gamma(\alpha)} \frac{\Gamma(2-\alpha-\epsilon) \Gamma(3-\epsilon)}{\Gamma(5-\alpha-2 \epsilon)}\right] .
\end{aligned}
$$

Here, the first fraction in the terms containing $\Gamma$ functions corresponds to the loop-momentum integration possibly resulting in UV singularities, while the second fraction corresponds to the integration over Feynman parameters and consequently, to collinear singularities. For $\alpha<2+\tilde{\epsilon}$, where $\tilde{\epsilon}=0$ or $\ll$, only the UV singularities appear, while for $\alpha>2+\tilde{\epsilon}$, only the collinear singularities appear. The two terms in the bracket cancel in both cases, so $I_{[\alpha \neq 2+\tilde{\epsilon}], \tilde{\epsilon} \ll}=0$. However, for $\alpha=2+\tilde{\epsilon}$, both UV and collinear singularities are present, and the cancellation can occur only if we abandon distinguishing them. origin, we obtain the following expression:

$$
\begin{aligned}
-i(4 \pi)^{2}\left[\mu^{2 \epsilon} \int \frac{d^{D} l}{(2 \pi)^{D}} \frac{1}{\left(l^{2}+i \eta\right)^{2}}\right] \\
=\left[\Gamma_{U V}(\epsilon) \frac{1}{1-2 \epsilon}\left(1-\frac{\epsilon}{2}\right)\right. \\
\left.\quad+\Gamma_{I R}(\epsilon) \frac{1}{1-2 \epsilon}\left(1-\frac{\epsilon}{2}\right)\right]\left(\frac{\mu^{2}}{\widetilde{Q}^{2}}\right)^{\epsilon},
\end{aligned}
$$

where $\tilde{Q}^{2}>0$ represents the auxiliary scale.

The NLO contribution $\tilde{\phi}^{(1)}(u, t)$, to which Feynman diagrams of Fig. 7 contribute, can then be expressed by

$$
\begin{aligned}
\tilde{\phi}^{(1)}(u, t)= & {\left[\Gamma_{U V}^{(0)}(\epsilon) \frac{1}{1-2 \epsilon}\left(1-\frac{\epsilon}{2}\right) K^{(1)}(u, t)\right.} \\
& \left.+\Gamma_{I R}^{(0)}(\epsilon) \frac{1}{1-2 \epsilon}\left(1-\frac{\epsilon}{2}\right) K^{(1)}(u, t)\right]\left(\frac{\mu^{2}}{\widetilde{Q}^{2}}\right)^{\epsilon},
\end{aligned}
$$

where the function $K^{(1)}$ calculated in the naive- $\gamma_{5}$ scheme amounts to

$$
\begin{aligned}
& K^{(1)}= 2 C_{F}\left\{\frac{u}{t}\left[(1-\epsilon)+\frac{1}{t-u}\right] \theta(t-u)\right. \\
&\left.+\left(\begin{array}{c}
u \rightarrow 1-u \\
t \rightarrow 1-t
\end{array}\right)\right\}_{+} . \\
& \text {3. } n_{f} \text {-proportional NNLO contributions }
\end{aligned}
$$

By inserting the vacuum polarizations in the NLO diagrams of Fig. 7, we obtain the NNLO diagrams displayed in Fig. 8. The $n_{f}$-proportional contributions ${ }^{4}$ of these diagrams, evaluated using Eqs. (4.13 4.14), read

\footnotetext{
4 There are more two-loop diagrams containing quark loops, but they contribute to the $n_{f}$-proportional NNLO part of the meson singlet distribution amplitude.
} 


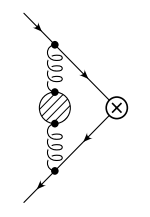

The function $f_{n_{f}}\left(\epsilon, \mu^{2}\right)$ is defined in (4.14b), while $K_{(X)_{n f}}$ is given by

$$
K_{(X)_{n f}}(u, t)=2 C_{F} \int_{0}^{1} d y y^{\epsilon} F_{X}(u, t ; y)
$$
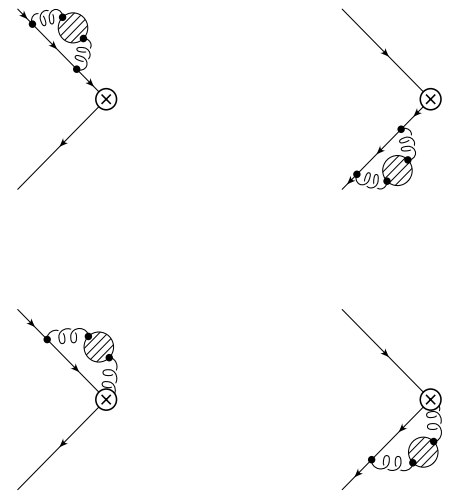

FIG. 8: Distinct two-loop Feynman diagrams contributing to the $\tilde{\phi}(u, t)$ distribution (3.6), i.e., (B3), which have been obtained by inserting the vacuum polarization bubbles in the one-loop diagrams of Fig. 6 .

$$
\begin{aligned}
& \tilde{\phi}_{(X)_{n f}}(u, t) \\
& =\frac{\alpha_{S}^{2}}{(4 \pi)^{2}}\left(-\frac{2}{3} n_{f}\right) K_{(X)_{n f}}(u, t)(1+\epsilon) f_{n_{f}}\left(\epsilon, \mu^{2}\right) \\
& \quad \times\left\{\frac{(4 \pi)^{2}}{i}\left[\mu^{2 \epsilon} \int \frac{d^{D} l}{(2 \pi)^{D}} \frac{1}{\left(l^{2}+i \eta\right)^{2+\epsilon}}\right]\right\} .
\end{aligned}
$$

and $F_{X}(u, t ; y)$ and $K_{(X)_{n f}}(u, t)$ can be read from Table III.

Similarly to (4.27), the $D$ dimensional integral from Eq. (4.30) gives

$$
\begin{gathered}
-i(4 \pi)^{2}\left[\mu^{2 \epsilon} \int \frac{d^{D} l}{(2 \pi)^{D}} \frac{1}{\left(l^{2}+i \eta\right)^{2+\epsilon}}\right] \\
=\frac{1}{\left(-\widetilde{Q}^{2}+i \eta\right)^{\epsilon}}\left(\frac{\mu^{2}}{\widetilde{Q}^{2}}\right)^{\epsilon} \\
\times\left[\Gamma_{U V}^{(1)}(\epsilon) \frac{1-\epsilon}{(1-3 \epsilon)\left(1-\frac{3}{2} \epsilon\right)} \frac{1-\frac{\epsilon}{2}}{1+\epsilon}\right. \\
\left.+\Gamma_{I R}^{(1)}(\epsilon) \frac{1-\epsilon}{(1-3 \epsilon)\left(1-\frac{3}{2} \epsilon\right)} \frac{1-\frac{\epsilon}{2}}{1+\epsilon}\right],
\end{gathered}
$$

where $\tilde{Q}^{2}>0$ represents the auxiliary scale.

The $n_{f}$-proportional NNLO contribution of the diagrams displayed in Fig. 8 takes the form

$$
\begin{aligned}
\tilde{\phi}^{\left(2, n_{f}\right)}(u, t)= & {\left[\Gamma_{U V}^{(0)}(\epsilon) \Gamma_{U V}^{(1)}(\epsilon) \frac{(1-\epsilon)^{2}\left(1-\frac{\epsilon}{2}\right)}{\left(1-\frac{2}{3} \epsilon\right)(1-2 \epsilon)(1-3 \epsilon)\left(1-\frac{3}{2} \epsilon\right)} K^{\left(2, n_{f}\right)}(u, t)\right.} \\
& \left.+\Gamma_{U V}^{(0)}(\epsilon) \Gamma_{I R}^{(1)}(\epsilon) \frac{(1-\epsilon)^{2}\left(1-\frac{\epsilon}{2}\right)}{\left(1-\frac{2}{3} \epsilon\right)(1-2 \epsilon)(1-3 \epsilon)\left(1-\frac{3}{2} \epsilon\right)} K^{\left(2, n_{f}\right)}(u, t)\right]\left(\frac{\mu^{2}}{\widetilde{Q}^{2}}\right)^{2 \epsilon}
\end{aligned}
$$

where the function $K^{\left(2, n_{f}\right)}$ calculated in the naive- $\gamma_{5}$ scheme amounts to

$$
\begin{aligned}
K^{\left(2, n_{f}\right)}(u, t)= & 2 C_{F}\left\{\frac{u}{t}\left[(1-\epsilon)+\frac{1}{t-u}\right]\left(\frac{u}{t}\right)^{\epsilon} \theta(t-u)\right. \\
& \left.+\left(\begin{array}{c}
u \rightarrow 1-u \\
t \rightarrow 1-t
\end{array}\right)\right\}_{+} .
\end{aligned}
$$

\section{The HV scheme results}

The preceding results have been calculated in the naive- $\gamma_{5}$ scheme. When the HV scheme is used, only the results for $A$ diagram from Fig. 7 and the corresponding "bubble" diagram from Fig. 8 differ from the naive- $\gamma_{5}$ results, and are given by

$$
\begin{aligned}
\tilde{\phi}_{A}^{H V}(u, t) & =\tilde{\phi}_{A}(u, t)+\Delta \tilde{\phi}_{A}(u, t) \\
& =\tilde{\phi}_{A}(u, t)\left(1+4 \epsilon \frac{1+\frac{\epsilon}{4}}{\left(1-\frac{\epsilon}{2}\right)(1-\epsilon)}\right),
\end{aligned}
$$


and

$$
\begin{aligned}
\tilde{\phi}_{(A)_{n f}}^{H V}(u, t) & =\tilde{\phi}_{(A)_{n f}}(u, t)+\Delta \tilde{\phi}_{(A)_{n f}}(u, t) \\
& =\tilde{\phi}_{(A)_{n f}}(u, t)\left(1+4 \epsilon \frac{1}{\left(1-\frac{\epsilon}{2}\right)(1-\epsilon)}\right) .
\end{aligned}
$$

Hence, when using the HV scheme, the functions $K^{(1)}$ and $K^{\left(2, n_{f}\right)}$ in Eqs. (4.28) and (4.33) get replaced by

$$
\begin{aligned}
K_{H V}^{(1)}= & 2 C_{F}\left\{4 \epsilon \frac { 1 + \frac { \epsilon } { 4 } } { 1 - \frac { \epsilon } { 2 } } \left[\frac{u}{t} \theta(t-u)\right.\right. \\
& \left.\left.+\left(\begin{array}{c}
u \rightarrow 1-u \\
t \rightarrow 1-t
\end{array}\right)\right]\right\}+K^{(1)},
\end{aligned}
$$

and

$$
\begin{aligned}
K_{H V}^{\left(2, n_{f}\right)}(u, t)= & 2 C_{F}\left\{4 \epsilon \frac { 1 } { 1 - \frac { \epsilon } { 2 } } \left[\frac{u}{t}\left(\frac{u}{t}\right)^{\epsilon} \theta(t-u)\right.\right. \\
& \left.\left.+\left(\begin{array}{c}
u \rightarrow 1-u \\
t \rightarrow 1-t
\end{array}\right)\right]\right\}+K^{\left(2, n_{f}\right)},
\end{aligned}
$$

respectively. These results, obviously, these results bear the signature of chiral symmetry violation.

\section{RENORMALIZATION AND FACTORIZATION OF COLLINEAR SINGULARITIES}

\section{A. General renormalization procedure}

Since in this work we present the calculation (up to $n_{f}$ proportional NNLO contributions) of the hard-scattering amplitude $T\left(u, Q^{2}\right)(4.1)$, as well as of the perturbatively calculable part of the DA $\tilde{\phi}(u, t)(4.22)$, both containing UV and collinear singularities, here we outline the general procedure for the renormalization of UV and the factorization of collinear singularities.

We introduce the amplitude $\mathcal{M}$ (having the same form of the perturbative expansion as the amplitudes $T\left(u, Q^{2}\right)$ and $\tilde{\phi}(u, t))$ :

$$
\mathcal{M}=\mathcal{M}^{(0)}+\frac{\alpha_{S}}{4 \pi} \mathcal{M}^{(1)}+\frac{\alpha_{S}^{2}}{(4 \pi)^{2}}\left[\left(-\frac{2}{3} n_{f}\right) \mathcal{M}^{\left(2, n_{f}\right)}+\cdots\right]+\cdots
$$

where

$$
\begin{aligned}
\mathcal{M}^{(1)}= & {\left[\Gamma_{U V}^{(0)}(\epsilon)\left(a_{0}^{U V}+\epsilon a_{1}^{U V}+\epsilon^{2} a_{2}^{U V}+O\left(\epsilon^{3}\right)\right)\right.} \\
& \left.+\Gamma_{I R}^{(0)}(\epsilon)\left(a_{0}^{I R}+\epsilon a_{1}^{I R}+\epsilon^{2} a_{2}^{I R}+O\left(\epsilon^{3}\right)\right)\right]\left(\frac{\mu^{2}}{Q^{2}}\right)^{\epsilon}, \\
\mathcal{M}^{\left(2, n_{f}\right)}= & {\left[\Gamma_{U V}^{(0)}(\epsilon) \Gamma_{U V}^{(1)}(\epsilon)\left(b_{0}^{n_{f}, U V}+\epsilon b_{1}^{n_{f}, U V}+\epsilon^{2} b_{2}^{n_{f}, U V}+O\left(\epsilon^{3}\right)\right)\right.} \\
& \left.+\Gamma_{U V}^{(0)}(\epsilon) \Gamma_{I R}^{(1)}(\epsilon)\left(b_{0}^{n_{f}, I R}+\epsilon b_{1}^{n_{f}, I R}+\epsilon^{2} b_{2}^{n_{f}, I R}+O\left(\epsilon^{3}\right)\right)\right]\left(\frac{\mu^{2}}{Q^{2}}\right)^{2 \epsilon},
\end{aligned}
$$

with $a_{i}^{U V(I R)}$ and $b_{i}^{U V(I R)}$ representing general coefficients in the expansion over $\epsilon$.

As a first step, we perform the coupling constant renormalization in the $\overline{M S}$ renormalization scheme. Note that in the functions $\Gamma_{U V}^{(0)}(\epsilon)$ and $\Gamma_{I R}^{(0)}(\epsilon)$, defined by (4.7), the singularities are contained in

$$
\begin{aligned}
\Gamma(\epsilon) \Gamma(1-\epsilon) & =\frac{\pi}{\sin \pi \epsilon}=\frac{1}{\epsilon}+\frac{\pi^{2}}{6} \epsilon+O\left(\epsilon^{3}\right) \\
& =-\Gamma(-\epsilon) \Gamma(1+\epsilon),
\end{aligned}
$$

while the remaining artifacts of dimensional regularization can be found in

$$
\frac{\Gamma(1-\epsilon)}{\Gamma(1-2 \epsilon)}(4 \pi)^{\epsilon}=1+\epsilon(-\gamma+\ln (4 \pi))+O\left(\epsilon^{2}\right)
$$

(and similarly for $\Gamma_{U V}^{(1)}$ and $\Gamma_{I R}^{(1)}$ functions $(4.16)$ ). By expanding $\Gamma$ functions over $\epsilon$, in relation (5.1), an unnecessary complication of keeping track of various $\gamma, \pi^{2}$, and $\ln 4 \pi$ terms, would be introduced. Instead, we make use of the freedom in defining the $\overline{M S}$ scheme beyond 
$O\left(\epsilon^{0}\right)$, which is explained in detail in Appendix 9 (along with some other conventions and "misconventions"), and define the bare coupling constant $\alpha_{S}$ in terms of the running coupling $\alpha_{S}\left(\mu_{R}^{2}\right)$ by

$\alpha_{S}=\left(\frac{\mu_{R}^{2}}{\mu^{2}}\right)^{\epsilon}\left(\epsilon \Gamma_{U V}^{(0)}(\epsilon)\right)^{-1} \alpha_{S}\left(\mu_{R}^{2}\right)\left(1-\frac{\alpha_{S}\left(\mu_{R}^{2}\right)}{4 \pi} \beta_{0} \frac{1}{\epsilon}\right)$

where $\beta_{0}=11-2 / 3 n_{f}$. The inclusion of the factor $\epsilon \Gamma_{U V}^{(0)}(\epsilon)$ in $(5.3)$ turns out to be very suitable for this type of calculation, in which both UV and IR singularities are regularized by the dimensional regularization method. The elegance and advantage introduced in the calculation by the choice (5.3) becomes clear when one notes that

$$
\begin{aligned}
\Gamma_{U V}^{(1)}(\epsilon) & =\frac{1}{2} \Gamma_{U V}^{(0)}(\epsilon)+O\left(\epsilon^{2}\right) \\
\Gamma_{I R}^{(1)}(\epsilon) & =\frac{1}{2} \Gamma_{I R}^{(0)}(\epsilon)+O\left(\epsilon^{2}\right)
\end{aligned}
$$

So, one can see that the presence of the factor $\epsilon \Gamma_{U V}^{(0)}(\epsilon)$ in (5.3) is natural in the sense that it contains the combinations of $\Gamma$ 's that naturally emerge in this calculation, and leads to their cancellation without expanding the whole result over $\epsilon$. That is in contrast to "artificial" choices like $\exp (\epsilon(-\gamma+\ln 4 \pi))$ and $(4 \pi)^{\epsilon} / \Gamma(1-\epsilon)$ found in the literature (for example, 27, 28 and [29, respectively).

By substituting (5.4) into (5.1), and performing the coupling constant renormalization according to (5.3), one obtains

$$
\mathcal{M}=\mathcal{M}^{(0)}+\frac{\alpha_{S}\left(\mu_{R}^{2}\right)}{4 \pi} \hat{\mathcal{M}}^{(1)}+\frac{\alpha_{S}^{2}\left(\mu_{R}^{2}\right)}{(4 \pi)^{2}}\left[\left(-\frac{2}{3} n_{f}\right) \hat{\mathcal{M}}_{n_{f}}^{(2)}+\cdots\right]+\cdots,
$$

where

$$
\begin{aligned}
\hat{\mathcal{M}}^{(1)}= & {\left[\frac{1}{\epsilon}\left(a_{0}^{U V}+\epsilon a_{1}^{U V}+O\left(\epsilon^{2}\right)\right)+\frac{1}{-\epsilon}\left(a_{0}^{I R}+\epsilon a_{1}^{I R}+O\left(\epsilon^{2}\right)\right)\right]\left(\frac{\mu_{R}^{2}}{Q^{2}}\right)^{\epsilon} } \\
\hat{\mathcal{M}}^{\left(2, n_{f}\right)}= & {\left[\frac { 1 } { \epsilon ^ { 2 } } \left(\left(\frac{b_{0}^{n_{f}, U V}}{2}-\left(\frac{\mu_{R}^{2}}{Q^{2}}\right)^{-\epsilon} a_{0}^{U V}\right)+\epsilon\left(\frac{b_{1}^{n_{f}, U V}}{2}-\left(\frac{\mu_{R}^{2}}{Q^{2}}\right)^{-\epsilon} a_{1}^{U V}\right)\right.\right.} \\
& \left.+\epsilon^{2}\left(\frac{b_{2}^{n_{f}, U V}}{2}-\left(\frac{\mu_{R}^{2}}{Q^{2}}\right)^{-\epsilon} a_{2}^{U V}\right)+O^{\prime}\left(\epsilon^{3}\right)\right) \\
+ & \frac{1}{-\epsilon^{2}}\left(\left(\frac{b_{0}^{n_{f}, I R}}{2}-\left(\frac{\mu_{R}^{2}}{Q^{2}}\right)^{-\epsilon} a_{0}^{I R}\right)+\epsilon\left(\frac{b_{1}^{n_{f}, I R}}{2}-\left(\frac{\mu_{R}^{2}}{Q^{2}}\right)^{-\epsilon} a_{1}^{I R}\right)\right. \\
& \left.\left.+\epsilon^{2}\left(\frac{b_{2}^{n_{f}, I R}}{2}-\left(\frac{\mu_{R}^{2}}{Q^{2}}\right)^{-\epsilon} a_{2}^{I R}\right)+O^{\prime}\left(\epsilon^{3}\right)\right)\right]\left(\frac{\mu_{R}^{2}}{Q^{2}}\right)^{2 \epsilon} .
\end{aligned}
$$

Note that the only artifact of dimensional regularization we are left with is the dimensional parameter $\epsilon$. The result (5.5) is given in a simple and compact form in which all terms in the expansion over $\epsilon$ are still retained. Also, the distinction between the singularities of UV and IR origin is still preserved.

If the coefficients of the $1 / \epsilon$ and $1 / \epsilon^{2}$ poles of UV origin are different from zero, the additional renormalization should be performed, as in the case of the multiplicatively renormalizable composite operator from $\hat{\phi}(u, t)$ (3.6). The UV singularities are then factorized in the renormalization constant $Z_{\mathcal{M}, \text { ren }}$. After all UV divergences are properly renormalized, the remaining $1 /(-\epsilon)$ and $1 /\left(-\epsilon^{2}\right)$ collinear poles should be, at some factorization scale, factorized in $Z_{\mathcal{M}, c o l}$.

\section{B. Renormalization of the hard-scattering amplitude $T$}

We shall now apply the results of the preceding subsection to the renormalization of our results for the hardscattering amplitude $T$ given by Eqs. (4.1), 4.5), (4.10), and, (4.21). By comparing (4.1) and (5.12), we can identify $\mathcal{M} \equiv T\left(u, Q^{2}\right) /\left(N_{T} / Q^{2}\right)$ and $\mathcal{M}^{(i)} \equiv T^{(i)}(u)$.

The NLO coefficients $a_{j}^{U V(I R)}$ appearing in $5.1 \mathrm{~b}$ ) are determined from (4.10) by expanding the coefficients of $\Gamma_{U V, I R}^{(0)}(\epsilon)$ over $\epsilon$, while the $n_{f}$-proportional NNLO coefficients $b_{j}^{n f, U V(I R)}$ in $5.1 \mathrm{C}$ ) are obtained from (4.21) by expanding the coefficients of $\Gamma_{U V}^{(0)}(\epsilon) \Gamma_{U V, I R}^{(1)}(\epsilon)$ over $\epsilon$. The 
TABLE IV: The NLO coefficients $a_{j}^{U V(I R)}$ defined in (5.1b) and determined for $\mathcal{M}^{(1)} \equiv T^{(1)}(u)(4.10)$. The $n_{f}$-proportional NNLO coefficients $b_{j}^{n f, U V(I R)}$ defined in 5.10 and determined for $\mathcal{M}^{\left(2, n_{f}\right)} \equiv T^{\left(2, n_{f}\right)}(u)$ (4.21).

\begin{tabular}{|c|c|}
\hline$a_{0}^{U V}$ & 0 \\
\hline$a_{1}^{U V}$ & $C_{F} \frac{1}{1-u}\left(\frac{1}{2}+\frac{1}{u} \ln (1-u)\right)+(u \rightarrow 1-u)$ \\
\hline$a_{2}^{U V}$ & $C_{F} \frac{1}{1-u}\left(\frac{1}{2}-\left(\frac{1}{2}-\frac{1}{u}\right) \ln (1-u)-\frac{1}{2 u} \ln ^{2}(1-u)\right)+(u \rightarrow 1-u)$ \\
\hline$\overline{a_{0}^{I R}}$ & $C_{F} \frac{1}{1-u}(3+2 \ln (1-u))+(u \rightarrow 1-u)$ \\
\hline$a_{1}^{I R}$ & $C_{F} \frac{1}{1-u}\left(\frac{19}{2}+\left(-(8 \delta-7)+\frac{8 \delta-6}{u}\right) \ln (1-u)-\ln ^{2}(1-u)\right)+(u \rightarrow 1-u)$ \\
\hline$a_{2}^{I R}$ & $\begin{array}{l}C_{F} \frac{1}{1-u}\left(\frac{37}{2}+\left(-\frac{40 \delta-29}{2}+\frac{20 \delta-15}{u}\right) \ln (1-u)+\left(\frac{8 \delta-7}{2}-\frac{8 \delta-6}{2 u}\right) \ln ^{2}(1-u)\right. \\
\left.+\frac{1}{3} \ln ^{3}(1-u)\right)+(u \rightarrow 1-u)\end{array}$ \\
\hline$\overline{b_{0}^{n_{f}, U V}}$ & 0 \\
\hline$b_{1}^{n_{f}, U V}$ & $C_{F} \frac{1}{1-u}\left(1+\frac{2}{u} \ln (1-u)\right)+(u \rightarrow 1-u)$ \\
\hline$b_{2}^{n_{f}, U V}$ & $C_{F} \frac{1}{1-u}\left(\frac{11}{3}-\left(2-\frac{25}{3 u}\right) \ln (1-u)-\frac{2}{u} \ln ^{2}(1-u)+\frac{2}{u} \operatorname{Li}_{2}(u)\right)+(u \rightarrow 1-u)$ \\
\hline$\overline{b_{0}^{n_{f}, I R}}$ & $C_{F} \frac{1}{1-u}(3+2 \ln (1-u))+(u \rightarrow 1-u)$ \\
\hline$b_{1}^{n_{f}, I R}$ & $\begin{array}{l}C_{F} \frac{1}{1-u}\left(\frac{39}{2}+\left(-\frac{24 \delta-34}{3}+\frac{24 \delta-18}{3 u}\right) \ln (1-u)-2 \ln ^{2}(1-u)+2 \operatorname{Li}_{2}(u)\right) \\
+(u \rightarrow 1-u)\end{array}$ \\
\hline$b_{2}^{n_{f}, I R}$ & $\begin{array}{l}C_{F} \frac{1}{1-u}\left(\frac{311}{4}+\left(-\frac{408 \delta-347}{9}+\frac{408 \delta-270}{9 u}\right) \ln (1-u)\right. \\
+\left(\frac{24 \delta-34}{3}-\frac{24 \delta-18}{3 u}\right) \ln ^{2}(1-u)+\frac{4}{3} \ln ^{3}(1-u) \\
\left.+\left(-\frac{24 \delta-52}{3}+\frac{24 \delta-18}{3 u}\right) \operatorname{Li}_{2}(u)-2 \operatorname{Li}_{3}(u)+4 S_{1,2}(u)\right)+(u \rightarrow 1-u)\end{array}$ \\
\hline
\end{tabular}

special cases of the generalized Nielsen polylogarithms

$$
\begin{aligned}
\operatorname{Li}_{2}(u) & =S_{1,1}(u)=-\int_{0}^{u} d x \frac{\ln (1-x)}{x} \\
\operatorname{Li}_{3}(u) & =S_{2,1}(u)=\int_{0}^{u} d x \frac{\operatorname{Li}_{2}(x)}{x} \\
S_{1,2}(u) & =\frac{1}{2} \int_{0}^{u} d x \frac{\ln ^{2}(1-x)}{x}
\end{aligned}
$$

appear in these results, and the useful identity is

$$
\begin{aligned}
S_{1,2}(u)= & -\operatorname{Li}_{3}(1-u)-\ln (1-u) \operatorname{Li}_{2}(u) \\
& -\frac{1}{2} \ln (u) \ln ^{2}(1-u)+\frac{\pi^{2}}{6} \ln (1-u)+\xi(3) .
\end{aligned}
$$

So, after the coupling constant renormalization has been performed, the hard-scattering amplitude
$T /\left(N_{T} / Q^{2}\right)$ takes the form given by (5.5), where the LO contribution $T^{(0)}$ is given by 4.5 and the coefficients $a_{j}^{U V(I R)}$ and $b_{j}^{n f, U V(I R)}$ are listed in Table IV. As expected, the coefficients of the UV poles in (5.5) vanish, since all UV singularities get removed by the coupling constant renormalization. According to Eq. (3.1), i.e.,

$$
T\left(u, Q^{2}\right)=T_{H}\left(x, Q^{2}, \mu_{F}^{2}\right) \otimes Z_{T, c o l}\left(x, u ; \mu_{F}^{2}\right),
$$

the remaining singularities of the collinear type factorize 
at the factorization scale $\mu_{F}^{2}$ in

$$
\begin{aligned}
& Z_{T, c o l}\left(x, u ; \mu_{F}^{2}\right) \\
& =\delta(x-u)+\frac{\alpha_{S}\left(\mu_{R}^{2}\right)}{4 \pi}\left(\frac{\mu_{R}^{2}}{\mu_{F}^{2}}\right)^{\epsilon} \frac{1}{-\epsilon} \tilde{a}_{0}^{I R}(x, u) \\
& +\frac{\alpha_{S}^{2}\left(\mu_{R}^{2}\right)}{(4 \pi)^{2}}\left(\frac{\mu_{R}^{2}}{\mu_{F}^{2}}\right)^{2 \epsilon} \frac{1}{-\epsilon^{2}}\left\{\left(-\frac{2}{3} n_{f}\right)\right. \\
& \times\left[\left(\frac{\tilde{b}_{0}^{n_{f}, I R}(x, u)}{2}-\left(\frac{\mu_{R}^{2}}{\mu_{F}^{2}}\right)^{-\epsilon} \tilde{a}_{0}^{I R}(x, u)\right)\right. \\
& \left.\left.+\epsilon\left(\frac{\tilde{b}_{1}^{n_{f}, I R}(x, u)}{2}-\tilde{a}_{1}^{I R}(x, u)\right)\right]+\cdots\right\}+\cdots
\end{aligned}
$$

where the coefficients $\tilde{a}_{i}^{I R}(x, u)$ and $\tilde{b}_{i}^{n_{f}, I R}(x, u)$ satisfy the relations

$$
\begin{aligned}
T^{(0)}(x) \otimes \tilde{a}_{i}^{I R}(x, u) & =a_{i}^{I R}(u), \\
T^{(0)}(x) \otimes \tilde{b}_{i}^{n_{f}, I R}(x, u) & =b_{i}^{n_{f}, I R}(u) .
\end{aligned}
$$

With the help of

$$
\begin{aligned}
& \alpha_{S}\left(\mu_{R}^{2}\right) \\
& =\left(\frac{\mu_{F}^{2}}{\mu_{R}^{2}}\right)^{\epsilon} \alpha_{S}\left(\mu_{F}^{2}\right)\left[1+\frac{\alpha_{S}\left(\mu_{F}^{2}\right)}{4 \pi} \beta_{0} \frac{1}{\epsilon}\left(\left(\frac{\mu_{F}^{2}}{\mu_{R}^{2}}\right)^{\epsilon}-1\right)\right],
\end{aligned}
$$

one can easily demonstrate that $Z_{T, c o l}\left(x, u ; \mu_{F}^{2}\right)$ is indeed independent of the hard-scattering renormalization scale $\mu_{R}^{2}$ :

$$
\begin{aligned}
& Z_{T, c o l}\left(x, u ; \mu_{F}^{2}\right) \\
& =\delta(x-u)+\frac{\alpha_{S}\left(\mu_{F}^{2}\right)}{4 \pi} \frac{1}{-\epsilon} \tilde{a}_{0}^{I R}(x, u) \\
& +\frac{\alpha_{S}^{2}\left(\mu_{F}^{2}\right)}{(4 \pi)^{2}} \frac{1}{-\epsilon^{2}}\left\{\left(-\frac{2}{3} n_{f}\right)\right. \\
& \times\left[\left(\frac{\tilde{b}_{0}^{n_{f}, I R}(x, u)}{2}-\tilde{a}_{0}^{I R}(x, u)\right)\right. \\
& \left.\left.\quad+\epsilon\left(\frac{\tilde{b}_{1}^{n_{f}, I R}(x, u)}{2}-\tilde{a}_{1}^{I R}(x, u)\right)\right]+\cdots\right\}+\cdots
\end{aligned}
$$

After factorizing the collinear singularities from (5.5) by (5.8), and taking into account that $b_{0}^{n_{f}, I R}=a_{0}^{I R}$, we obtain

$$
\begin{aligned}
\frac{T_{H}\left(x, Q^{2}, \mu_{F}^{2}\right)}{N_{T} / Q^{2}}= & T^{(0)}(x)+\frac{\alpha_{S}\left(\mu_{R}^{2}\right)}{4 \pi}\left[\left(a_{1}^{U V}(x)-a_{1}^{I R}(x)\right)-a_{0}^{I R}(x) \ln \frac{\mu_{F}^{2}}{Q^{2}}+O(\epsilon)\right] \\
& +\frac{\alpha_{S}^{2}\left(\mu_{R}^{2}\right)}{(4 \pi)^{2}}\left\{( - \frac { 2 } { 3 } n _ { f } ) \left[\left(\frac{b_{2}^{n_{f}, U V}(x)}{2}-\frac{b_{2}^{n_{f}, I R}(x)}{2}\right)-\left(a_{2}^{U V}(x)-a_{2}^{I R}(x)\right)\right.\right. \\
& +\left(a_{1}^{U V}(x)-a_{1}^{I R}(x)-a_{0}^{I R}(x) \ln \frac{\mu_{F}^{2}}{Q^{2}}\right) \ln \frac{\mu_{R}^{2}}{Q^{2}} \\
& \left.\left.-\left(b_{1}^{n_{f}, I R}(x)-2 a_{1}^{I R}(x)\right) \ln \frac{\mu_{F}^{2}}{Q^{2}}+\frac{1}{2} a_{0}^{I R}(x) \ln ^{2} \frac{\mu_{F}^{2}}{Q^{2}}+O(\epsilon)\right]+\cdots\right\} \\
& +\cdots
\end{aligned}
$$

where the $O(\epsilon)$ terms can now be safely neglected (notice that we have kept all $\epsilon^{n}$ terms till the end of calculation).

Finally, having evaluated all the necessary terms, we summarize our result for the hard-scattering amplitude $T_{H}\left(x, Q^{2}, \mu_{F}^{2}\right)$ in the form

$$
\begin{aligned}
T_{H}\left(x, Q^{2}, \mu_{F}^{2}\right)= & T_{H}^{(0)}\left(x, Q^{2}\right)+\frac{\alpha_{S}\left(\mu_{R}^{2}\right)}{4 \pi} T_{H}^{(1)}\left(x, Q^{2}, \mu_{F}^{2}\right) \\
& +\frac{\alpha_{S}^{2}\left(\mu_{R}^{2}\right)}{(4 \pi)^{2}}\left[\left(-\frac{2}{3} n_{f}\right) T_{H}^{\left(2, n_{f}\right)}\left(x, Q^{2}, \mu_{R}^{2}, \mu_{F}^{2}\right)+\cdots\right]+\cdots,
\end{aligned}
$$


where

$$
\begin{aligned}
T_{H}^{(0)}\left(x, Q^{2}\right)= & \frac{N_{T}}{Q^{2}} A^{(0)}(x)+(x \rightarrow 1-x) \\
T_{H}^{(1)}\left(x, Q^{2}, \mu_{F}^{2}\right)= & \frac{N_{T}}{Q^{2}}\left(A^{(1)}(x)-A_{c o l}^{(1)}(x) \ln \frac{\mu_{F}^{2}}{Q^{2}}\right)+(x \rightarrow 1-x) \\
T_{H}^{\left(2, n_{f}\right)}\left(x, Q^{2}, \mu_{R}^{2}, \mu_{F}^{2}\right)= & \frac{N_{T}}{Q^{2}}\left[A^{\left(2, n_{f}\right)}(x)+\left(A^{(1)}(x)-A_{c o l}^{(1)}(x) \ln \frac{\mu_{F}^{2}}{Q^{2}}\right) \ln \frac{\mu_{R}^{2}}{Q^{2}}\right. \\
& \left.-A_{c o l}^{\left(2, n_{f}\right)}(x) \ln \frac{\mu_{F}^{2}}{Q^{2}}+\frac{1}{2} A_{c o l}^{(1)}(x) \ln ^{2} \frac{\mu_{F}^{2}}{Q^{2}}\right]+(x \rightarrow 1-x) .
\end{aligned}
$$

We have introduced the functions $A^{(i)}$ and $A_{c o l}^{(i)}$, which are given by

$$
A^{(0)}(x)=\frac{1}{1-x},
$$

and

$$
\begin{aligned}
A^{(1)}(x)= & C_{F} \frac{1}{1-x}\left[-9-(8 \delta-7) \frac{1-x}{x} \ln (1-x)+\ln ^{2}(1-x)\right], \\
A^{\left(2, n_{f}\right)}(x)= & C_{F} \frac{1}{1-x}\left[-\frac{457}{24}+\left(\frac{(48 \delta-95)}{18}+\frac{(-16 \delta+19)}{6 x}\right) \ln (1-x)\right. \\
& +\left(\frac{13}{6}-\frac{1}{2 x}\right) \ln ^{2}(1-x)-\frac{1}{3} \ln ^{3}(1-x) \\
& \left.+\left(\frac{(12 \delta-26)}{3}-\frac{(4 \delta-4)}{x}\right) \operatorname{Li}_{2}(x)+\operatorname{Li}_{3}(x)-2 S_{1,2}(x)\right],
\end{aligned}
$$

while

$$
\begin{aligned}
A_{c o l}^{(1)}(x) & =C_{F} \frac{1}{1-x}(3+2 \ln (1-x)) \\
A_{c o l}^{\left(2, n_{f}\right)}(x) & =C_{F} \frac{1}{1-x}\left[\frac{1}{2}+\left(\frac{(24 \delta-8)}{3}+\frac{(-8 \delta+6)}{x}\right) \ln (1-x)+2 \operatorname{Li}_{2}(x)\right] .
\end{aligned}
$$

The collinearly singular terms removed from 5.5 by (5.8) correspond to

$$
\begin{aligned}
& T_{H}^{(0)}\left(x, Q^{2}\right) \otimes Z_{T, c o l}\left(x, u ; \mu_{F}^{2}\right) \\
& =\frac{N_{T}}{Q^{2}}\left\{A^{(0)}(u)+\frac{\alpha_{S}\left(\mu_{F}^{2}\right)}{4 \pi} \frac{1}{-\epsilon} A_{c o l}^{(1)}(u)+\frac{\alpha_{S}^{2}\left(\mu_{F}^{2}\right)}{(4 \pi)^{2}} \frac{1}{-\epsilon^{2}}\right. \\
& \quad \times\left[\left(-\frac{2}{3} n_{f}\right)\left(-\frac{1}{2} A_{c o l}^{(1)}(u)+\epsilon \frac{1}{2} A_{c o l}^{\left(2, n_{f}\right)}(u)\right)+\cdots\right] \\
& \quad+\cdots\}+(u \rightarrow 1-u) .
\end{aligned}
$$

The functions $A_{c o l}^{(i)}(5.15)$, which appear in $(5.16)$ and as coefficients of $\ln ^{n}\left(\mu_{F}^{2} / Q^{2}\right)$ in (5.13), are obviously connected to collinear singularities of the hard-scattering amplitude $T$.

\section{Renormalization of the perturbatively calculable DA part}

\section{General analysis}

Next, we proceed to renormalize the $\tilde{\phi}(u, t)$ following the procedure outlined in subsection $\mathrm{VA}$. By comparing (5.1) with (4.22) we identify $\mathcal{M} \equiv \phi(u, t)$ and $\mathcal{M}^{(i)} \equiv$ $\bar{\phi}^{(i)}(u, t)$, while the $Q^{2}$ scale corresponds to the scale $\tilde{Q}^{2}$. The coefficients

$$
\begin{aligned}
a_{i}^{U V} & =a_{i}^{I R} \equiv a_{i}(u, t) \\
b_{i}^{n_{f}, U V} & =b_{i}^{n_{f}, I R} \equiv b_{i}^{n_{f}}(u, t)
\end{aligned}
$$

are determined from Eqs. (4.28) and (4.29) by expanding the coefficients of $\Gamma_{U V, I R}^{(0)}(\epsilon)$ over $\epsilon$, and from Eqs. (4.33) and (4.34) by expanding the coefficients of 
$\Gamma_{U V}^{(0)}(\epsilon) \Gamma_{U V, I R}^{(1)}(\epsilon)$ over $\epsilon$, respectively. Although in this work only the $n_{f}$-proportional part of the NNLO contribution $\tilde{\phi}_{n_{f}}^{(2)}(u, t)$ has been determined, our symbolical analysis can be extended to the whole NNLO contribution $\tilde{\phi}^{(2)}(u, t)$. In this case the general coefficients of the order $\alpha_{S}^{2} \operatorname{term} \tilde{\phi}^{(2)}(u, t) \equiv \mathcal{M}^{(2)}$

$$
b_{i}^{U V}=b_{i}^{I R} \equiv b_{i}(u, t)
$$

appear. After the coupling constant renormalization, the distribution amplitude $\tilde{\phi}(u, t) \equiv \mathcal{M}$ is given by the expression (5.5) with the renormalization scale denoted by $\tilde{\mu}_{R}^{2}$. The complete order $\alpha_{S}^{2}\left(\tilde{\mu}_{R}^{2}\right)$ coefficient $\hat{\mathcal{M}}^{(2)}$ reads

$$
\begin{aligned}
\hat{\mathcal{M}}^{(2)}= & {\left[\frac { 1 } { \epsilon ^ { 2 } } \left(\left(\frac{b_{0}}{2}-\beta_{0}\left(\frac{\tilde{\mu}_{R}^{2}}{\tilde{Q}^{2}}\right)^{-\epsilon} a_{0}\right)+\epsilon\left(\frac{b_{1}}{2}-\beta_{0}\left(\frac{\tilde{\mu}_{R}^{2}}{\tilde{Q}^{2}}\right)^{-\epsilon} a_{1}\right)\right.\right.} \\
& \left.+\epsilon^{2}\left(\frac{b_{2}}{2}-\beta_{0}\left(\frac{\tilde{\mu}_{R}^{2}}{\tilde{Q}^{2}}\right)^{-\epsilon} a_{2}\right)+O^{\prime}\left(\epsilon^{3}\right)\right) \\
+ & \frac{1}{-\epsilon^{2}}\left(\left(\frac{b_{0}}{2}-\beta_{0}\left(\frac{\tilde{\mu}_{R}^{2}}{\tilde{Q}^{2}}\right)^{-\epsilon} a_{0}\right)+\epsilon\left(\frac{b_{1}}{2}-\beta_{0}\left(\frac{\tilde{\mu}_{R}^{2}}{\tilde{Q}^{2}}\right)^{-\epsilon} a_{1}\right)\right. \\
& \left.\left.+\epsilon^{2}\left(\frac{b_{2}}{2}-\beta_{0}\left(\frac{\tilde{\mu}_{R}^{2}}{\tilde{Q}^{2}}\right)^{-\epsilon} a_{2}\right)+O^{\prime}\left(\epsilon^{3}\right)\right)\right]\left(\frac{\tilde{\mu}_{R}^{2}}{\tilde{Q}^{2}}\right)^{2 \epsilon},
\end{aligned}
$$

where Eqs. 5.17 5.18 have already been taken into account.

As denoted in (3.8)

$$
\begin{aligned}
& \tilde{\phi}(u, t) \\
& \quad=Z_{\phi, r e n}\left(u, v ; \tilde{\mu}_{R}^{2}\right) \otimes \phi_{V}\left(v, s ; \tilde{\mu}_{R}^{2}, \mu_{0}^{2}\right) \otimes Z_{\phi, c o l}\left(s, t ; \mu_{0}^{2}\right) .
\end{aligned}
$$

the remaining UV singularities are multiplicatively renormalizable and factorize in the renormalization constant $Z_{\phi, r e n}\left(u, v ; \tilde{\mu}_{R}^{2}\right)$ given by

$$
\begin{aligned}
Z_{\phi, r e n}= & \mathbb{1}+\frac{\alpha_{S}\left(\tilde{\mu}_{R}^{2}\right)}{4 \pi} \frac{1}{\epsilon} a_{0}+\frac{\alpha_{S}^{2}\left(\tilde{\mu}_{R}^{2}\right)}{(4 \pi)^{2}} \frac{1}{\epsilon^{2}} \\
& \times\left[\left(\frac{b_{0}}{2}-\beta_{0} a_{0}\right)+\epsilon\left(\frac{b_{1}}{2}-\beta_{0} a_{1}-a_{0} a_{1}\right)\right] \\
& +\cdots,
\end{aligned}
$$

with

$$
b_{0}-\beta_{0} a_{0}-a_{0}^{2}=0
$$

(i.e., $\left.b_{0}(x, y)-\beta_{0} a_{0}(x, y)-a_{0}(x, u) \otimes a_{0}(u, y)=0\right)$ emerging as the condition of multiplicative renormalizability. As for the collinear singularities, they factorize at the factorization scale $\mu_{0}^{2}$ in $Z_{\phi, c o l}\left(s, t ; \mu_{0}^{2}\right)$ given by

$$
\begin{aligned}
& Z_{\phi, c o l} \\
& =\mathbb{1}+\frac{\alpha_{S}\left(\mu_{0}^{2}\right)}{4 \pi} \frac{1}{-\epsilon} a_{0}+\frac{\alpha_{S}^{2}\left(\mu_{0}^{2}\right)}{(4 \pi)^{2}} \frac{1}{-\epsilon^{2}} \\
& \times\left[\left(\frac{b_{0}}{2}-\beta_{0} a_{0}-a_{0}^{2}\right)+\epsilon\left(\frac{b_{1}}{2}-\beta_{0} a_{1}-a_{0} a_{1}\right)\right] \\
& \quad+\cdots .
\end{aligned}
$$

Finally, based on Eqs. 3.8 and 5.20 5.22, the function $\phi_{V}\left(v, s ; \tilde{\mu}_{R}^{2}, \mu_{0}^{2}\right)$ is obtained. It is free of singularities, and after the $\epsilon \rightarrow 0$ limit is taken, it takes the form

$$
\begin{aligned}
\phi_{V}= & \mathbb{1}+\frac{\alpha_{S}\left(\tilde{\mu}_{R}^{2}\right)}{4 \pi} a_{0} \ln \frac{\tilde{\mu}_{R}^{2}}{\mu_{0}^{2}}+\frac{\alpha_{S}^{2}\left(\tilde{\mu}_{R}^{2}\right)}{(4 \pi)^{2}} \\
& \times\left[\frac{b_{0}}{2} \ln ^{2} \frac{\tilde{\mu}_{R}^{2}}{\mu_{0}^{2}}+\left(b_{1}-2 \beta_{0} a_{1}-2 a_{0} a_{1}\right) \ln \frac{\tilde{\mu}_{R}^{2}}{\mu_{0}^{2}}\right] \\
& +\cdots
\end{aligned}
$$

Note that the auxiliary scale $\tilde{Q}^{2}$ has disappeared after renormalization and factorization of collinear singularities. We can make a distinction between the scale $\tilde{\mu}_{R, 1}^{2}$ introduced by the coupling constant renormalization and the scale $\tilde{\mu}_{R, 2}^{2}$ at which the remaining UV singularities are factorized in the renormalization constant $Z_{\phi, \text { ren }}$. It can be easily shown that the scale $\tilde{\mu}_{R, 1}^{2}$ vanishes from the end results, i.e., that $Z_{\phi, r e n}$ and $\phi_{V}$ depend only on the scale $\tilde{\mu}_{R, 2}^{2}$. Hence, $\tilde{\mu}_{R} \equiv \tilde{\mu}_{R, 2}^{2}$ and $\mu_{0}^{2}$ are the only relevant scales. Also, note that $Z_{\phi, \text { col }}\left(\mu^{2}\right)=Z_{\phi, r e n}^{-1}\left(\mu^{2}\right)$, which is expected, since, in dimensional regularization, $\tilde{\phi}=\mathbb{1}$ when the distinction between UV and collinear singularities is abandoned.

\section{Remarks on the evolutional part of the DA}

As explained in Sec. II, the function $\tilde{\phi}(u, t)$ represents a perturbatively calculable part of the unrenormalized pion distribution amplitude $\Phi(u)$. By taking into account Eqs. (3.7) and (3.8) the distribution $\Phi(u)$ can be 
expressed by 3.9

$$
\Phi(u)=Z_{\phi, r e n}\left(u, v ; \tilde{\mu}_{R}^{2}\right) \otimes \phi_{V}\left(v, s ; \tilde{\mu}_{R}^{2}, \mu_{0}^{2}\right) \otimes \Phi\left(s, \mu_{0}^{2}\right),
$$

where $\Phi\left(s, \mu_{0}^{2}\right)$ represents the pion distribution amplitude determined at the scale $\mu_{0}^{2}$. Its evolution to the scale $\tilde{\mu}_{R}^{2}$ is determined by $\phi_{V}\left(v, s ; \tilde{\mu}_{R}^{2}, \mu_{0}^{2}\right)$ and given by (3.11):

$$
\Phi\left(v, \tilde{\mu}_{R}^{2}\right)=\phi_{V}\left(v, s ; \tilde{\mu}_{R}^{2}, \mu_{0}^{2}\right) \otimes \Phi\left(s, \mu_{0}^{2}\right) .
$$

The evolution potential $V$ defined in (2.5) can be obtained from (3.5)

$$
V=-Z_{\phi, r e n}^{-1}\left(\tilde{\mu}_{R}^{2} \frac{\partial}{\partial \tilde{\mu}_{R}^{2}} Z_{\phi, r e n}\right),
$$

using (5.20), and it reads

$$
V=\frac{\alpha_{S}\left(\tilde{\mu}_{R}^{2}\right)}{4 \pi} V_{1}+\frac{\alpha_{S}^{2}\left(\tilde{\mu}_{R}^{2}\right)}{(4 \pi)^{2}} V_{2}+\cdots,
$$

where

$$
\begin{aligned}
& V_{1}=a_{0} \\
& V_{2}=b_{1}-2 \beta_{0} a_{1}-2 a_{0} a_{1} .
\end{aligned}
$$

By noting that

$$
\frac{\alpha_{S}\left(\tilde{\mu}_{R}^{2}\right)}{4 \pi} \ln \frac{\tilde{\mu}_{R}^{2}}{\mu_{0}^{2}}=\frac{1}{\beta_{0}}\left(1-\frac{\alpha_{S}\left(\tilde{\mu}_{R}^{2}\right)}{\alpha_{S}\left(\mu_{0}^{2}\right)}\right)=O\left(\alpha_{S}^{0}\right)
$$

and by employing the multiplicative renormalizability condition (5.21) as well as the results (5.24b), the $\alpha_{S}$ expansion of $\phi_{V}$ given in (5.23) can be reorganized and written in the form

$$
\phi_{V}=\phi_{V}^{L O}+\frac{\alpha_{S}\left(\tilde{\mu}_{R}^{2}\right)}{4 \pi} \phi_{V}^{N L O}+\cdots,
$$

where

$$
\begin{aligned}
\phi_{V}^{L O}= & \mathbb{1}+\frac{\alpha_{S}\left(\tilde{\mu}_{R}^{2}\right)}{4 \pi} \ln \frac{\tilde{\mu}_{R}^{2}}{\mu_{0}^{2}} V_{1} \\
& +\frac{\alpha_{S}^{2}\left(\tilde{\mu}_{R}^{2}\right)}{(4 \pi)^{2}} \ln ^{2} \frac{\tilde{\mu}_{R}^{2}}{\mu_{0}^{2}} \frac{1}{2}\left(V_{1}^{2}+\beta_{0} V_{1}\right)+\cdots
\end{aligned}
$$

and

$$
\phi_{V}^{N L O}=\frac{\alpha_{S}\left(\tilde{\mu}_{R}^{2}\right)}{4 \pi} \ln \frac{\tilde{\mu}_{R}^{2}}{\mu_{0}^{2}} V_{2}+\cdots
$$

denote the LO and NLO part, respectively. By substituting (5.26a) into (3.11) one obtains

$$
\begin{aligned}
\Phi\left(v, \tilde{\mu}_{R}^{2}\right) \\
=\phi_{V}^{L O}\left(v, s ; \tilde{\mu}_{R}^{2}, \mu_{0}^{2}\right) \otimes \Phi\left(s, \mu_{0}^{2}\right) \\
\quad+\frac{\alpha_{S}\left(\tilde{\mu}_{R}^{2}\right)}{4 \pi} \phi_{V}^{N O}\left(v, s ; \tilde{\mu}_{R}^{2}, \mu_{0}^{2}\right) \otimes \Phi\left(s, \mu_{0}^{2}\right)+\cdots \\
=\Phi^{L O}\left(v, \tilde{\mu}_{R}^{2}\right)+\frac{\alpha_{S}\left(\tilde{\mu}_{R}^{2}\right)}{4 \pi} \Phi^{N L O}\left(v, \tilde{\mu}_{R}^{2}\right)+\cdots .
\end{aligned}
$$

As it is seen from (5.26b) and 5.26c), the results of the two-loop calculation correspond to the first terms of the LO and NLO contributions to the $\phi_{V}$ function.

The complete LO and NLO behavior of $\phi_{V}\left(v, s ; \tilde{\mu}_{R}^{2}\right)$ and, consequently, of $\Phi\left(v, \tilde{\mu}_{B}^{2}\right)$ can be determined by solving the evolution equation (2.5), or equivalently (3.12)

$$
\begin{aligned}
& \tilde{\mu}_{R}^{2} \frac{\partial}{\partial \tilde{\mu}_{R}^{2}} \phi_{V}\left(v, s, \tilde{\mu}_{R}^{2}, \mu_{0}^{2}\right) \\
& \quad=V\left(v, s^{\prime}, \tilde{\mu}_{R}^{2}\right) \otimes \phi_{V}\left(s^{\prime}, s, \tilde{\mu}_{R}^{2}, \mu_{0}^{2}\right) .
\end{aligned}
$$

The LO result is of the form

$$
\begin{aligned}
\phi_{V}^{L O}\left(v, s ; \tilde{\mu}_{R}^{2}\right)= & \sum_{n=0}^{\infty}, \frac{v(1-v)}{N_{n}} C_{n}^{3 / 2}(2 v-1) \\
& \times C_{n}^{3 / 2}(2 s-1)\left(\frac{\alpha_{S}\left(\tilde{\mu}_{R}^{2}\right)}{\alpha_{S}\left(\mu_{0}^{2}\right)}\right)^{-\gamma_{n}^{(0)} / \beta_{0}},
\end{aligned}
$$

where $N_{n}=(n+1)(n+2) /(4(2 n+3))$, and $C_{n}^{3 / 2}(2 x-1)$ are the Gegenbauer polynomials representing the eigenfunctions of the LO kernel $V_{1}$ with the corresponding eigenvalues

$$
\gamma_{n}^{(0)}=C_{F}\left[3+\frac{2}{(n+1)(n+2)}-4 \sum_{i=1}^{n+1} \frac{1}{i}\right] .
$$

One can show the agreement between the complete LO prediction given above and the expansion (5.26b). The complete formal solution of the NLO evolution equation was obtained in 23] making use of conformal constraints, and the form of $\phi_{V}^{N L O}$ can be extracted from the results listed in $[8]$.

\section{Analytical results up to $n_{f}$-proportional NNLO terms (obtained using the naive- $\gamma_{5}$ scheme)}

After this lengthy general analysis we now turn to displaying the results. Using the multiplicative renormalizability condition (5.21) and the notation (5.24b), the renormalization constant $Z_{\phi, r e n}\left(u, v ; \tilde{\mu}_{R}^{2}\right)$ from Eq. (5.20) is expressed by

$$
\begin{aligned}
& Z_{\phi, \text { ren }}\left(u, v ; \tilde{\mu}_{R}^{2}\right) \\
& =\delta(u-v)+\frac{\alpha_{S}\left(\tilde{\mu}_{R}^{2}\right)}{4 \pi} \frac{1}{\epsilon} V_{1}(u, v)+\frac{\alpha_{S}^{2}\left(\tilde{\mu}_{R}^{2}\right)}{(4 \pi)^{2}} \frac{1}{\epsilon^{2}} \\
& \quad \times\left[\left(-\frac{2}{3} n_{f}\right)\left(\frac{-V_{1}(u, v)}{2}+\epsilon \frac{V_{2}^{n_{f}}(u, v)}{2}\right)+\cdots\right] \\
& \quad+\cdots .
\end{aligned}
$$

Here we list only the relevant combinations of $a_{i}, b_{i}$ coefficients:

$$
\begin{aligned}
& V_{1}(u, t)=a_{0}(u, t) \\
& =2 C_{F}\left\{\frac{u}{t}\left[1+\frac{1}{t-u}\right] \theta(t-u)+\left(\begin{array}{c}
u \rightarrow 1-u \\
t \rightarrow 1-t
\end{array}\right)\right\}_{+},
\end{aligned}
$$




$$
\begin{aligned}
& V_{2}^{n_{f}}(u, t)=b_{1}^{n_{f}}(u, t)-2 a_{1}(u, t) \\
& =2 C_{F}\left\{\frac{u}{t}\left[1+\left(1+\frac{1}{t-u}\right)\left(\frac{5}{3}+\ln \frac{u}{t}\right)\right] \theta(t-u)\right. \\
& \left.\quad+\left(\begin{array}{c}
u \rightarrow 1-u \\
t \rightarrow 1-t
\end{array}\right)\right\}_{+}
\end{aligned}
$$

Our results confirm the well-known form of the one-loop kernel $V_{1}$ [2] and the two-loop $n_{f}$-proportional kernel $V_{2}^{n_{f}}$ [19, 20, 21]. For later use we also specify the convolution of the functions given in (5.31) with the frequently encountered $1 /(1-x)$ term:

$$
\frac{1}{1-x} \otimes V_{1}(x, u)=C_{F} \frac{1}{1-u}(3+2 \ln (1-u))
$$

and

$$
\begin{aligned}
& \frac{1}{1-x} \otimes V_{2}^{n_{f}}(x, u) \\
& =C_{F} \frac{1}{1-u}\left[\frac{1}{2}+\left(\frac{16}{3}-\frac{2}{u}\right) \ln (1-u)+2 \operatorname{Li}_{2}(u)\right]
\end{aligned}
$$

\section{The results obtained using the $H V$ scheme}

In the following we present the results obtained using the HV scheme.

The function $\tilde{\phi}$ calculated in the naive- $\gamma_{5}$ scheme and the function $\tilde{\phi}^{H V}$ obtained in the HV scheme are related by

$$
\tilde{\phi}=\mathcal{Z}_{H V, U V}^{-1} \tilde{\phi}^{H V} \mathcal{Z}_{H V, c o l}^{-1}
$$

The factors $\mathcal{Z}_{H V, U V}^{-1}$ and $\mathcal{Z}_{H V, \text { col }}^{-1}$ remove the "spurious" anomalous terms introduced by the presence of dimensionally regulated UV and collinear singularities, respectively.

The renormalization of the $\tilde{\phi}^{H V}$ function proceeds analogously to the renormalization of $\tilde{\phi}$ described in preceding subsection. While

$$
a_{0}^{H V}=a_{0}, \quad b_{0}^{H V}=b_{0},
$$

the coefficients $a_{i}, b_{i}$ for $i \geq 1$ get replaced by

$$
\begin{aligned}
a_{i}^{H V} & =a_{i}+\Delta a_{i} \\
b_{i}^{H V} & =b_{i}+\Delta b_{i},
\end{aligned}
$$

and, according to 3.8 , the UV and collinear singularities are factorized

$$
\tilde{\phi}^{H V}=Z_{\phi, r e n}^{H V} \phi_{V}^{H V} Z_{\phi, c o l}^{H V} .
$$

By comparing $Z_{\phi, r e n}^{H V}, Z_{\phi, c o l}^{H V}$, and $\phi_{V}^{H V}$ with the results 5.20 5.23 obtained using the naive- $\gamma_{5}$ scheme, Eq. (5.36) takes the form

$$
\begin{aligned}
\tilde{\phi}^{H V}= & \left(Z_{\phi, r e n} \mathcal{Z}_{H V, U V}^{\text {div }}\right)\left(\mathcal{Z}_{H V, U V}^{\text {fin }} \phi_{V} \mathcal{Z}_{H V, c o l}^{\text {fin }}\right) \\
& \times\left(\mathcal{Z}_{H V, c o l}^{\text {div }} Z_{\phi, c o l}\right),
\end{aligned}
$$

where

$$
\begin{aligned}
\mathcal{Z}_{H V}^{d i v} & \equiv \mathcal{Z}_{H V, U V}^{d i v} \\
= & \mathbb{1}+\frac{\alpha_{S}^{2}\left(\tilde{\mu}_{R}^{2}\right)}{(4 \pi)^{2}} \frac{1}{\epsilon}\left(\frac{\Delta b_{1}}{2}-\beta_{0} \Delta a_{1}-a_{0} \Delta a_{1}\right) \\
& +\cdots
\end{aligned}
$$

and

$$
\begin{aligned}
\mathcal{Z}_{H V}^{f i n} & \equiv \mathcal{Z}_{H V, U V}^{f i n} \\
= & \mathbb{1}+\frac{\alpha_{S}\left(\tilde{\mu}_{R}^{2}\right)}{4 \pi}\left(\Delta a_{1}+O(\epsilon)\right)+\frac{\alpha_{S}^{2}\left(\tilde{\mu}_{R}^{2}\right)}{(4 \pi)^{2}} \\
& \times\left(\frac{\Delta b_{1}}{2}-\beta_{0} \Delta a_{2}-a_{0} \Delta a_{2}-a_{1} \Delta a_{1}+O(\epsilon)\right) \\
& +\cdots,
\end{aligned}
$$

while $\mathcal{Z}_{H V, \text { col }}^{\text {div, }}\left(\mu^{2}\right)=\left(\mathcal{Z}_{H V, U V}^{\text {div,fin }}\right)^{-1}\left(\mu^{2}\right)$. The condition of multiplicative renormalizability of the "spurious" anomalous terms introduced by the HV scheme reads

$$
\Delta b_{1}-\beta_{0} \Delta a_{1}-2 a_{0} \Delta a_{1}=0
$$

Finally, we list the results obtained by substituting $K_{H V}^{(1)}$ and $K_{n_{f}, H V}^{(2)} 4.374 .38$ in place of $K^{(1)}$ and $K^{(2)}$ in Eqs. 4.224 .33 . The combinations of the $\Delta a_{i}$ and $\Delta b_{i}$ coefficients, which appear in (5.38 5.42) after 5.40) is taken into account, read

$$
\Delta a_{1}(u, t)=2 C_{F}\left\{4 \frac{u}{t} \theta(t-u)+\left(\begin{array}{c}
u \rightarrow 1-u \\
t \rightarrow 1-t
\end{array}\right)\right\}
$$

$$
\begin{aligned}
& \Delta b_{1}^{n_{f}}(u, t)-2 \Delta a_{2}(u, t) \\
& =2 C_{F}\left\{\frac{u}{t}\left(\frac{8}{3}+4 \ln \frac{u}{t}\right) \theta(t-u)+\left(\begin{array}{c}
u \rightarrow 1-u \\
t \rightarrow 1-t
\end{array}\right)\right\} .
\end{aligned}
$$

The complete renormalization constant $\mathcal{Z}_{H V} \equiv \mathcal{Z}_{H V, U V}$ from Eq. (5.33) is then given by

$$
\mathcal{Z}_{H V}\left(u, v ; \tilde{\mu}_{R}^{2}\right)=\mathcal{Z}_{H V}^{d i v}\left(u, w ; \tilde{\mu}_{R}^{2}\right) \otimes \mathcal{Z}_{H V}^{f i n}\left(w, v ; \tilde{\mu}_{R}^{2}\right) .
$$

By utilizing the condition of multiplicative renormalizability of the "spurious" terms (5.40), it takes the form 


$$
\begin{aligned}
\mathcal{Z}_{H V}\left(u, v ; \tilde{\mu}_{R}^{2}\right)= & \delta(u-v)+\frac{\alpha_{S}\left(\tilde{\mu}_{R}^{2}\right)}{4 \pi}\left(\Delta a_{1}(u, v)+O(\epsilon)\right) \\
& +\frac{\alpha_{S}^{2}\left(\tilde{\mu}_{R}^{2}\right)}{(4 \pi)^{2}}\left[\left(-\frac{2}{3} n_{f}\right)\left(\frac{1}{\epsilon} \frac{-\Delta a_{1}(u, v)}{2}+\frac{\Delta b_{1}^{n_{f}}(u, v)-2 \Delta a_{2}(u, v)}{2}+O(\epsilon)\right)+\cdots\right] \\
& +\cdots
\end{aligned}
$$

It is interesting to note that by using the "reduction" formulas, which relate the exclusive ("nonforward") and inclusive ("forward") kernels [21], the agreement between the renormalization constant $\mathcal{Z}_{H V}$ given above and an analogous "HV" renormalization constant for the longitudinal spin structure function $g_{1}$ [31] is established (up to $O(\epsilon)$ terms).

For later use we specify the convolution of functions defined in Eq. (5.41) with the 1/(1-x) term:

$$
\frac{1}{1-x} \otimes \Delta a_{1}(x, u)=C_{F} \frac{1}{1-u}\left(-8 \frac{1-u}{u} \ln (1-u)\right),
$$

and

$$
\begin{aligned}
& \frac{1}{1-x} \otimes\left(\Delta b_{1}^{n_{f}}(x, u)-2 \Delta a_{2}(x, u)\right) \\
& =C_{F} \frac{1}{1-u}\left(-\frac{16}{3} \frac{1-u}{u} \ln (1-u)-8 \frac{1-u}{u} \operatorname{Li}_{2}(u)\right) .
\end{aligned}
$$

\section{THE EXPRESSION FOR THE PION TRANSITION FORM FACTOR UP TO $n_{f}$-PROPORTIONAL NNLO TERMS}

We now combine the results for the hard-scattering amplitude and the DA obtained in the preceding sections. After resolving the $\gamma_{5}$ problem and discussing the dependence of the prediction on the factorization scale $\mu_{F}^{2}$, we finally present the expression for the pion transition form factor up to $n_{f}$-proportional NNLO terms.

\section{A. Resolving the $\gamma_{5}$ ambiguity}

In subsections $\mathrm{VB}$ and $\mathrm{VC}$, we have presented the results of the perturbative treatment of the hard-scattering amplitude $T\left(u, Q^{2}\right)$ and the distribution amplitude $\Phi(u)$, respectively. Along the lines outlined in Sec. III, we now proceed to combine these results to obtain the finite and $\gamma_{5}$-scheme independent expression for the pion transition form factor $F_{\gamma^{*} \gamma \pi}\left(Q^{2}\right)$, up to the $n_{f}$-proportional NNLO contributions.

The lack of the ambiguity in the DA results along with the fact that the prediction for the pion transition form factor should not depend on the choice of the $\gamma_{5}$-scheme make it possible to resolve the ambiguity of the $\gamma_{5}$ treatment in the hard-scattering calculation.

\section{Naive $\gamma_{5}$-scheme}

The appearance of two $\gamma_{5}$ matrices imposes the use of the naive- $\gamma_{5}$ scheme in the DA calculation and the corresponding results are presented in (5.26 5.31). The $\gamma_{5}$ matrix present in the hard-scattering amplitude can also be treated in the naive- $\gamma_{5}$ scheme in which case a number of results emerge. After the Ward identities of QED are taken into account, the remaining ambiguity in the hard-scattering amplitude result (5.12-5.16) is parameterized by the parameter $\delta$ (as explained in Appendix A and Subsec. IV A).

Matching Eqs. (5.15) and (5.32) one observes that

$$
\begin{aligned}
\frac{1}{1-x} \otimes V_{1}(x, u) & =A_{c o l}^{(1)}(u) \\
\frac{1}{1-x} \otimes V_{2}^{n_{f}}(x, u) & =\left.A_{c o l}^{\left(2, n_{f}\right)}(u)\right|_{\delta=1} .
\end{aligned}
$$

If these relations are taken into account in Eq. (5.16), then a comparison with (5.30), for $\tilde{\mu}_{R}^{2}=\mu_{F}^{2}$ (i.e., for the DA $\Phi(u)$ renormalized at the $\mu_{F}^{2}$ scale), gives

$$
\left.Z_{T, c o l}\right|_{\delta=1}=Z_{\phi, \text { ren }}^{-1}
$$

i.e., the relation (3.14) is satisfied and the singularities in (3.13) cancel for the $\delta$ parameter taking the value 1 . Hence, we obtain

$$
\left.Z_{T, c o l}\left(x, u ; \mu_{F}^{2}\right) \equiv Z_{T, c o l}\left(x, u ; \mu_{F}^{2}\right)\right|_{\delta=1},
$$

and, consequently,

$$
\left.T_{H}\left(x, Q^{2}, \mu_{F}^{2}\right) \equiv T_{H}\left(x, Q^{2}, \mu_{F}^{2}\right)\right|_{\delta=1},
$$

where Eq. (6.2), together with (5.30), determines $\left.Z_{T . c o l}\left(x, u ; \mu_{F}^{2}\right)\right|_{\delta=1}$, while taking $\delta=1$ in Eqs. (5.12 5.15) gives $\left.T_{H}\left(x, Q^{2}, \mu_{F}^{2}\right)\right|_{\delta=1}$. Hereby, we have confirmed the $\gamma_{5}$ prescription employed in [10] (see Appendix A for further details).

In preceding consideration, we have resolved the $\gamma_{5}$ ambiguity of the hard-scattering prediction by adopting the naive $\gamma_{5}$-scheme and by using the unambiguous DA results (along with the QED Ward identities) to single out the correct prediction. 


\section{HV scheme}

Let us now present the calculation performed in the HV scheme. Continuing to $D$ dimensions and adopting the HV scheme leads to unique results, but the "spurious" anomalous terms, which violate chiral symmetry, appear and additional renormalization is required, both for the DA and the hard-scattering amplitude.

The results for the hard-scattering amplitude obtained in the HV scheme correspond to the $\delta=0$ choice in 5.12 5.16), and the notation $T^{H V}, T_{H}^{H V}, Z_{T, \text { col }}^{H V}$ has been introduced. The fact that the UV singularities appearing in the hard-scattering amplitude get completely renormalized by the coupling constant renormalization indicates that, contrary to the DA case, only the presence of collinear singularities along with the nonanticommuting nature of $\gamma_{5}$ matrix introduces "spurious" anomalous terms.

The corresponding renormalization constant for the DA denoted by $\mathcal{Z}_{H V}=\mathcal{Z}_{H V}^{\text {div }} \mathcal{Z}_{H V}^{\text {fin }}$ and displayed in 5.38 5.43 has been determined by comparing the "correct" results obtained in the naive- $\gamma_{5}$ with the corresponding results obtained using the $\mathrm{HV}$ scheme. As a result, one finds that the unrenormalized DA in the $\mathrm{HV}$ scheme, $\Phi^{H V}\left(u^{\prime}\right)$, and the unrenormalized DA in the naive- $\gamma_{5}$ scheme, $\Phi(u)$, are related by

$$
\Phi=\mathcal{Z}_{H V}^{-1} \Phi^{H V}
$$

Similarly, the renormalization constants $Z_{\phi, \text { ren }}$ and $Z_{\phi, \text { ren }}^{H V}$ determined in the naive- $\gamma_{5}$ and the HV schemes, respectively, are related by

$$
Z_{\phi, r e n}=\mathcal{Z}_{H V}^{\text {div }}{ }^{-1} Z_{\phi, r e n}^{H V}
$$

while the additional finite "HV" renormalization of the renormalized distribution $\Phi^{H V}\left(v^{\prime}, \tilde{\mu}_{R}^{2}\right)$ calculated in the $\mathrm{HV}$ scheme, is needed to obtain the renormalized DA $\Phi\left(v, \tilde{\mu}_{R}^{2}\right)$ free of "spurious" anomalies:

$$
\Phi=\mathcal{Z}_{H V}^{f i n-1} \Phi^{H V}
$$

The prediction for the pion transition form factor cannot depend on the choice of the scheme and chiral symmetry is restored for the complete result, i.e.,

$$
\begin{aligned}
F_{\gamma^{*} \gamma \pi} & =T \Phi^{\dagger}=T \mathcal{Z}_{H V}^{-1} \mathcal{Z}_{H V} \Phi^{\dagger} \\
& =T^{H V} \Phi^{H V \dagger}
\end{aligned}
$$

or equivalently

$$
\begin{aligned}
F_{\gamma^{*} \gamma \pi} & =T_{H} \Phi^{*}=T_{H}\left(\mathcal{Z}_{H V}^{f i n}\right)^{-1} \mathcal{Z}_{H V}^{f i n} \Phi^{*} \\
& =T_{H}^{H V} \Phi^{H V *}
\end{aligned}
$$

On the basis of Eqs. 5.44) and (5.385.43), along with Eqs. (5.125.16), it can easily be shown that

$$
T=T^{H V} \mathcal{Z}_{H V}
$$

while

$$
T_{H}=T_{H}^{H V} \mathcal{Z}_{H V}^{f i n}
$$

and

$$
Z_{T, \text { col }}=Z_{T, \text { col }}^{H V} \mathcal{Z}_{H V}^{d i v}
$$

with $T_{H}$ and $Z_{T, \text { col }}$ being given by Eqs. (6.4) and (6.3), respectively, and $T=T_{H} Z_{T, \text { col }}$.

Hence, we have resolved the $\gamma_{5}$ ambiguity appearing in the hard-scattering amplitude calculation by consistently treating, in either the naive- $\gamma_{5}$ or the HV scheme, both the hard-scattering amplitude and the distribution amplitude (which, actually, is free of the $\gamma_{5}$ ambiguity).

\section{B. Discussing the factorization scale dependence}

After the $\gamma_{5}$ ambiguity is resolved, and the collinear singularities present in the $T\left(u, Q^{2}\right)$ and $\Phi(u)$ amplitudes cancel, we are left with the finite prediction for the pion transition form factor

$$
F_{\gamma^{*} \gamma \pi}\left(Q^{2}\right)=T_{H}\left(x, Q^{2}, \mu_{F}^{2}\right) \otimes \Phi^{*}\left(x, \mu_{F}^{2}\right) .
$$

The hard-scattering amplitude $T_{H}\left(x, Q^{2}, \mu_{F}^{2}\right)$, evaluated up to $n_{f}$-proportional NNLO terms, is given by Eqs. (5.12 5.15) with $\delta=1$.

The distribution amplitude $\Phi^{*}\left(x, \mu_{F}^{2}\right)$ is determined by evoluting $\Phi^{*}\left(x, \mu_{0}^{2}\right)$ (obtained at the scale $\mu_{0}^{2}$ using some nonperturbative method) to the scale $\mu_{F}^{2}$ according to (3.11)

$$
\Phi\left(v, \tilde{\mu}_{R}^{2}\right)=\phi_{V}\left(v, s ; \mu_{F}^{2}, \mu_{0}^{2}\right) \otimes \Phi\left(s, \mu_{0}^{2}\right),
$$

$$
\begin{aligned}
& \text { i.e., } 5.27 \text { ) } \\
& \Phi\left(v, \mu_{F}^{2}\right) \\
& =\phi_{V}^{L O}\left(v, s ; \mu_{F}^{2}, \mu_{0}^{2}\right) \otimes \Phi\left(s, \mu_{0}^{2}\right) \\
& +\frac{\alpha_{S}\left(\mu_{F}^{2}\right)}{4 \pi} \phi_{V}^{N L O}\left(v, s ; \mu_{F}^{2}, \mu_{0}^{2}\right) \otimes \Phi\left(s, \mu_{0}^{2}\right)+\cdots .
\end{aligned}
$$

In subsection $\mathrm{VC}$ we have analysed in detail the evolutional part $\phi_{V}$, and as noted there, the two-loop DA calculation explicitly gives only the first few terms of the LO and NLO contributions, while the complete LO and NLO behavior of the DA is determined by solving the evolution equation (2.5), or equivalently (3.12)

$$
\begin{aligned}
& \mu_{F}^{2} \frac{\partial}{\partial \mu_{F}^{2}} \phi_{V}\left(v, s, \mu_{F}^{2}, \mu_{0}^{2}\right) \\
& \quad=V\left(v, s^{\prime}, \mu_{F}^{2}\right) \otimes \phi_{V}\left(s^{\prime}, s, \mu_{F}^{2}, \mu_{0}^{2}\right) .
\end{aligned}
$$

The dependence of $T_{H}\left(x, Q^{2}, \mu_{F}^{2}\right)$ on the factorization scale $\mu_{F}^{2}$ can be determined analogously to the $\mu_{F}^{2}$ dependence of the DA. Thus, by differentiating Eq. (3.1) with respect to $\mu_{F}^{2}$ and taking into account Eqs. (3.5) and (3.14) one finds that $T_{H}\left(x, Q^{2}, \mu_{F}^{2}\right)$ satisfies the equation

$$
\mu_{F}^{2} \frac{\partial}{\partial \mu_{F}^{2}} T_{H}\left(x, Q^{2}, \mu_{F}^{2}\right)=-T_{H}\left(y, Q^{2}, \mu_{F}^{2}\right) \otimes V\left(y, x ; \mu_{F}^{2}\right),
$$


which, as it is seen, is analogous to the DA evolution equation (2.5). Therefore, just as in the case of the DA, any finite order solution of (6.14) contains the complete $\mu_{F}^{2}$ dependence of $T_{H}\left(x, Q^{2}, \mu_{F}^{2}\right)$ which is not the case with the expansion (5.12) truncated at the same order (and not taking into account (5.25)).

The hard-scattering amplitude $T_{H}\left(x, Q^{2}, \mu_{F}^{2}\right)$ can be written in the factorized form

$T_{H}\left(x, Q^{2}, \mu_{F}^{2}\right)=T_{H}\left(y, Q^{2}, \mu_{F}^{2}=Q^{2}\right) \otimes \phi_{V}\left(y, x, Q^{2}, \mu_{F}^{2}\right)$,

with $\phi_{V}\left(y, x, Q^{2}, \mu_{F}^{2}\right)$ containing all its $\mu_{F}^{2}$ dependence. This can easily be demonstrated if use is made of $T_{H}$ and $\phi_{V}$ determined up to $n_{f}$-proportional NNLO terms (see Eqs. (5.26), (5.32), and (C3b)). On the other hand, using Eq. (3.12) one can show by partial integration that 6.15) indeed represents the solution of the evolution equation (6.14).

When calculating to any finite order in $\alpha_{S}$, it is inappropriate to convolute the $\Phi\left(x, \mu_{F}^{2}\right)$ distribution obtained by solving the evolution equation (2.5) (i.e., given by (2.8 2.13) ) with $T_{H}\left(x, Q^{2}, \mu_{F}^{2}\right)$ obtained by the truncation of the expansion (5.12). Namely, in the latter function, only the partial dependence on $\mu_{F}^{2}$ is included, in contrast to the former. Notice that when the complete dependence of $T_{H}$ on $\mu_{F}^{2}$ is taken into account even the LO term in (6.15) is $\mu_{F}^{2}$ dependent, in contrast to $T^{(0)}$ given in (2.4), and this leads to $\mu_{F}^{2}$ independent LO prediction for the pion transition form factor.

Substituting Eqs. (6.15) and (3.11) into Eq. (6.13) and taking into account that

$$
\phi_{V}\left(y, x, Q^{2}, \mu_{F}^{2}\right) \otimes \phi_{V}\left(x, s, \mu_{F}^{2}, \mu_{0}^{2}\right)=\phi_{V}\left(y, s, Q^{2}, \mu_{0}^{2}\right),
$$

one obtains

$F_{\gamma^{*} \gamma \pi}\left(Q^{2}\right)=T_{H}\left(y, Q^{2}, Q^{2}\right) \otimes \phi_{V}\left(y, s, Q^{2}, \mu_{0}^{2}\right) \otimes \Phi^{*}\left(s, \mu_{0}^{2}\right)$
The relation (6.16) is valid at every order of perturbative calculation (to NLO this can easily be checked by substituting (5.28) and the NLO results of Ref. 23] into (6.16)). It represents the resummation of the $\ln \left(Q^{2} / \mu_{0}^{2}\right)$ logarithms over the intermediate $\mu_{F}^{2}$ scale, performed in such a way that, first, the logarithms $\ln \left(\mu_{F}^{2} / \mu_{0}^{2}\right)$ originating from the perturbative part of the DA are resummed, and then the summation of $\ln \left(Q^{2} / \mu_{F}^{2}\right)$ logarithms from the hard-scattering part is performed. Therefore, the summations of the $\mu_{F}^{2}$ logarithms can be accomplished with any choice of $\mu_{F}^{2}$, because the effect in the final prediction, at every order, is the same as if the complete renormalization-group resummation of $\ln \left(Q^{2} / \mu_{0}^{2}\right)$ logarithms has been performed.

Consequently, the $F_{\gamma^{*} \gamma \pi}$ prediction (2.2) (as well as the prediction for any other exclusive quantity obtained in the standard hard-scattering picture) is independent of the factorization scale $\mu_{F}^{2}$ at every order in $\alpha_{S}$, provided both $T_{H}$ and $\Phi$ are consistently treated regarding the $\mu_{F}^{2}$ dependence. The intermediate scale at which the short- and long-distance dynamics separate, the factorization scale $\mu_{F}^{2}$, disappears from the final prediction at every order in $\alpha_{S}$ and therefore does not introduce any theoretical uncertainty into the PQCD calculation for exclusive processes.

We would like to point out here that by adopting the common choice $\mu_{F}^{2}=Q^{2}$, one avoids the need for the resummation of the $\ln \left(Q^{2} / \mu_{F}^{2}\right)$ logarithms in the hard-scattering part, making the calculation simpler and hence, for practical purposes, the preferable form of $F_{\gamma^{*} \gamma \pi}\left(Q^{2}\right)$ is given by

$$
F_{\gamma^{*} \gamma \pi}\left(Q^{2}\right)=T_{H}\left(x, Q^{2}, Q^{2}\right) \otimes \Phi^{*}\left(x, Q^{2}\right)
$$

\section{Presenting the final results}

Finally, we summarize. Taking into account Eqs. 5.12 5.15) and the results of the preceding subsections, the hardscattering amplitude $T_{H}\left(x, Q^{2}, \mu_{F}^{2}=Q^{2}\right)$, free of all effects of collinear singularities (the terms containing functions $A_{c o l}^{(n)} \ln ^{n} \mu_{F}^{2} / Q^{2}$ factorized in $\left.\phi_{V}\right)$, takes the form

$$
\begin{aligned}
T_{H}\left(x, Q^{2}, \mu_{F}^{2}=Q^{2}\right)= & T_{H}^{(0)}\left(x, Q^{2}\right)+\frac{\alpha_{S}\left(\mu_{R}^{2}\right)}{4 \pi} T_{H}^{(1)}\left(x, Q^{2}, \mu_{F}^{2}=Q^{2}\right) \\
& +\frac{\alpha_{S}^{2}\left(\mu_{R}^{2}\right)}{(4 \pi)^{2}}\left[\left(-\frac{2}{3} n_{f}\right) T_{H}^{\left(2, n_{f}\right)}\left(x, Q^{2}, \mu_{F}^{2}=Q^{2}, \mu_{R}^{2}\right)+\cdots\right]+\cdots
\end{aligned}
$$


where

$$
\begin{aligned}
T_{H}^{(0)}\left(x, Q^{2}\right) & =\frac{N_{T}}{Q^{2}} A^{(0)}(x)+(x \rightarrow 1-x), \\
T_{H}^{(1)}\left(x, Q^{2}, \mu_{F}^{2}=Q^{2}\right) & =\frac{N_{T}}{Q^{2}} A^{(1)}(x)+(x \rightarrow 1-x), \\
T_{H}^{\left(2, n_{f}\right)}\left(x, Q^{2}, \mu_{F}^{2}=Q^{2}, \mu_{R}^{2}\right) & =\frac{N_{T}}{Q^{2}}\left(A^{\left(2, n_{f}\right)}(x)+A^{(1)}(x) \ln \frac{\mu_{R}^{2}}{Q^{2}}\right)+(x \rightarrow 1-x),
\end{aligned}
$$

and

$$
\begin{aligned}
A^{(0)}(x)= & \frac{1}{1-x}, \\
A^{(1)}(x)= & C_{F} \frac{1}{1-x}\left[-9-\frac{1-x}{x} \ln (1-x)+\ln ^{2}(1-x)\right], \\
A^{\left(2, n_{f}\right)}(x)= & C_{F} \frac{1}{1-x}\left[-\frac{457}{24}-\left(\frac{47}{18}-\frac{1}{2 x}\right) \ln (1-x)+\left(\frac{13}{6}-\frac{1}{2 x}\right) \ln ^{2}(1-x)\right. \\
& \left.-\frac{1}{3} \ln ^{3}(1-x)-\frac{14}{3} \operatorname{Li}_{2}(x)+\operatorname{Li}_{3}(x)-2 S_{1,2}(x)\right] .
\end{aligned}
$$

To obtain the distribution amplitude $\Phi\left(x, Q^{2}\right)$ one evolutes $\Phi\left(x, \mu_{0}^{2}\right)$, determined using some nonperturbative methods at the scale $\mu_{0}^{2}$, to the scale $Q^{2}$ according to (2.8.2.13).

By substituting Eqs. (6.19), (2.8), and (2.10) into (6.18) and taking $(\mathrm{C} 3 \mathrm{~b})$ into account the pion transition form factor $F_{\gamma^{*} \gamma \pi}$ expressed as a perturbative series in $\alpha_{S}\left(\mu_{R}^{2}\right)$ reads

$$
\begin{aligned}
F_{\gamma^{*} \gamma \pi} & \left(Q^{2}\right) \\
= & F_{\gamma^{*} \gamma \pi}^{(0)}\left(Q^{2}\right)+\frac{\alpha_{S}\left(\mu_{R}^{2}\right)}{4 \pi} F_{\gamma^{*} \gamma \pi}^{(1)}\left(Q^{2}\right) \\
& +\frac{\alpha_{S}^{2}\left(\mu_{R}^{2}\right)}{(4 \pi)^{2}}\left[\left(-\frac{2}{3} n_{f}\right) F_{\gamma^{*} \gamma \pi}^{\left(2, n_{f}\right)}\left(Q^{2}, \mu_{R}^{2}\right)+\cdots\right] \\
& +\cdots,
\end{aligned}
$$

where

$$
\begin{aligned}
F_{\gamma^{*} \gamma \pi}^{(0)}\left(Q^{2}\right)= & T_{H}^{(0)}\left(x, Q^{2}\right) \otimes \Phi^{L O}\left(x, Q^{2}\right), \\
F_{\gamma^{*} \gamma \pi}^{(1)}\left(Q^{2}\right)= & T_{H}^{(1)}\left(x, Q^{2}, Q^{2}\right) \otimes \Phi^{L O}\left(x, Q^{2}\right) \\
& +T_{H}^{(0)}\left(x, Q^{2}\right) \otimes \Phi^{N L O}\left(x, Q^{2}\right),
\end{aligned}
$$

$$
\begin{aligned}
F_{\gamma^{*} \gamma \pi}^{\left(2, n_{f}\right)}\left(Q^{2}, \mu_{R}^{2}\right)= & T_{H}^{\left(2, n_{f}\right)}\left(x, Q^{2}, Q^{2}, \mu_{R}^{2}\right) \otimes \Phi^{L O}\left(x, Q^{2}\right) \\
& +T_{H}^{(0)}\left(x, Q^{2}\right) \otimes \Phi^{N L O}\left(x, Q^{2}\right) \ln \frac{\mu_{R}^{2}}{Q^{2}}
\end{aligned}
$$

As it is seen, we are left with one expansion parameter: $\alpha_{S}\left(\mu_{R}^{2}\right)$. The scale $\mu_{R}^{2}$ actually represents the renormalization scale of the complete perturbatively calculable part of the pion transition form factor (6.17), i.e., of

$$
T_{H}\left(y, Q^{2}, \mu_{0}^{2}\right)=T_{H}\left(y, Q^{2}, Q^{2}\right) \otimes \phi_{V}\left(y, s, Q^{2}, \mu_{0}^{2}\right) .
$$

Although, the physical pion transition form factor $F_{\gamma^{*} \gamma \pi}\left(Q^{2}\right)$ does not depend on the choice of the renormalization scale $\mu_{R}^{2}$, when calculating to any finite order a residual dependence on the $\mu_{R}^{2}$ scale remains.

\section{NUMERICAL PREDICTIONS}

\section{A. Fixing the renormalization scale according to the BLM procedure}

The dependence of finite order predictions on the renormalization scale introduces a theoretical uncertainty in their interpretation (see [8] for a detailed discussion), which is especially evident in calculations to lowest order in order $\alpha_{S}$. It would be advantageous to optimize the scale choice according to some sensible criteria. The BLM procedure 13, 14, 15] offers such criteria. The essence of the BLM procedure is that all vacuum-polarization effects (gluon vacuum polarization contributions, analogous to QED, as well as, quark vacuum polarization and vertex corrections) from the $\mathrm{QCD}$ $\beta$ function are incorporated into the running coupling constant rather than into the coefficients of the perturbative expansion. In practice, this amounts to computing quark-loop insertions in the diagrams of that order (since $\left.\beta_{0}=11-2 / 3 n_{f}, \cdots\right)$ and setting the scale by demanding that the coefficients of the perturbative expansion are $n_{f}$ independent. 
Hence, according to the BLM scale setting prescription, the renormalization scale for the pion transition form factor entering at the NLO is determined from the NNLO $n_{f}$-proportional terms, and is fixed by the requirement

$$
F_{\gamma^{*} \gamma \pi}^{\left(2, n_{f}\right)}\left(Q^{2}, \mu_{R}^{2}\right)=0
$$

$\mu_{R}^{2}$ has been implicitly assumed, i.e., $\mu_{R}^{2}$ has been treated as independent of the momentum fractions $x$ throughout the paper; otherwise the factorization of singularities would take a cumbersome form (if menagable at all). Apart from that we would be faced with the problem of a clear separation of the short- and long- distance effects 35.

Note that in the present calculation the effective nature of

Therefore, the only consistent way to asses the BLM scale is to solve Eq. (7.1), resulting in some mean value BLM scale

$$
\mu_{R}^{2}=\mu_{B L M}^{2}=a_{B L M}\left(Q^{2}\right) Q^{2}
$$

where

$$
a_{B L M}\left(Q^{2}\right)=\exp \left(-\frac{A^{\left(2, n_{f}\right)}(x) \otimes \Phi_{L O}\left(x, Q^{2}\right)}{A^{(1)}(x) \otimes \Phi_{L O}\left(x, Q^{2}\right)+A^{(0)}(x) \otimes \Phi_{N L O}\left(x, Q^{2}\right)}\right) .
$$

As it is seen, the scale $\mu_{B L M}^{2}$ depends on the specific form of the distribution amplitude.

In Section II the nonperturbative input, i.e., the distribution amplitude $\phi\left(x, \mu_{0}^{2}\right)=\Phi\left(x, \mu_{0}\right) / N_{\phi}$ determined at the scale $\mu_{0}^{2}$, has been presented in the form of an expansion over Gegenbauer polynomials (2.10). The evolution to the scale $\mu_{F}^{2}$ has been described by Eqs. (2.11 2.13). Retaining only the first three terms in the general expansion of the pion DA given in Eq. (2.10)

$$
\phi\left(x, \mu_{0}^{2}\right)=6 x(1-x)\left[1+B_{2} C_{2}^{3 / 2}(2 x-1)+B_{4} C_{4}^{3 / 2}(2 x-1)\right],
$$

the LO and NLO contributions to $Q^{2} F_{\gamma^{*} \gamma \pi}\left(Q^{2}\right)$ take the form

$$
\begin{aligned}
& Q^{2} F_{\gamma^{*} \gamma \pi}^{(0)}\left(Q^{2}\right)=2 C_{\pi} f_{\pi}\left[3\left(1+B_{2}^{L O}\left(Q^{2}\right)+B_{4}^{L O}\left(Q^{2}\right)\right)\right] \\
& Q^{2} F_{\gamma^{*} \gamma \pi}^{(1)}\left(Q^{2}\right)=2 C_{\pi} f_{\pi}\left[\left(-20+\frac{295}{18} B_{2}^{L O}\left(Q^{2}\right)+\frac{10487}{225} B_{4}^{L O}\left(Q^{2}\right)\right)+3 \sum_{k=2}^{\infty} B_{k}^{N L O}\left(Q^{2}\right)\right]
\end{aligned}
$$

while the $n_{f}$ proportional NNLO contribution amounts to

$$
\begin{aligned}
Q^{2} F_{\gamma^{*} \gamma \pi}^{\left(2, n_{f}\right)}\left(Q^{2}\right)=2 C_{\pi} & f_{\pi}\left[\left(-43.47+78.47 B_{2}^{L O}\left(Q^{2}\right)+197.165 B_{4}^{L O}\left(Q^{2}\right)\right)\right. \\
+ & \left(-20+\frac{295}{18} B_{2}^{L O}\left(Q^{2}\right)+\frac{10487}{225} B_{4}^{L O}\left(Q^{2}\right)\right) \ln \frac{\mu_{R}^{2}}{Q^{2}} \\
+ & \left.\left(3 \sum_{k=2}^{\infty}{ }^{\prime} B_{k}^{N L O}\left(Q^{2}\right)\right) \ln \frac{\mu_{R}^{2}}{Q^{2}}\right] .
\end{aligned}
$$

Therefore, on the basis of Eqs. (7.1) and (7.6) one finds that the BLM scale for the pion transition form factor is given by (7.2a) with

$$
a_{B L M}\left(Q^{2}\right)=\exp \left(-\frac{-43.47+78.47 B_{2}^{L O}\left(Q^{2}\right)+197.165 B_{4}^{L O}\left(Q^{2}\right)}{-20+\frac{295}{18} B_{2}^{L O}\left(Q^{2}\right)+\frac{10487}{225} B_{4}^{L O}\left(Q^{2}\right)+3 \sum_{k=2}^{\infty}{ }^{N} B_{k}^{N O}\left(Q^{2}\right)}\right) .
$$

Expressions (6.22) and $(7.47 .6)$, representing the com-

plete NLO prediction for the pion transition form factor, 
together with the expressions (7.2a) and (7.7), specifying the corresponding BLM scale, are valid for arbitrary distribution amplitude (with the evolutional effects included) and represent the main results of this paper.

\section{B. Numerical predictions in the $\overline{M S}$ and $\alpha_{V}$ schemes}

Based on the general expressions derived in a preceding subsection, we now proceed to obtain numerical predictions for the pion transition form factor using two specific distribution amplitudes: the asymptotic DA and the CZ distribution amplitude.

There is increasing theoretical evidence coming from different calculations [32, 33, 34, 38] that the low energy pion distribution amplitude does not differ much from its asymptotic form $\phi_{a s}(x)=6 x(1-x)$ (which represents the solution of the evolution equation (2.5) for $\mu_{F}^{2} \rightarrow \infty$ ). The distribution $\phi\left(x, \mu_{0}^{2}\right)=\phi_{a s}(x)$ is characterized by the fact that at the LO it has no evolution, while the NLO evolutional effects are tiny [8], and for the purpose of this calculation these effects can safely be neglected.

The expression for the pion transition form factor $Q^{2} F_{\gamma^{*} \gamma \pi}\left(Q^{2}\right)$, based on the Eqs. (6.22) and (7.4 7.6), and corresponding to the asymptotic distribution then reads

$$
\begin{aligned}
& Q^{2} F_{\gamma^{*} \gamma \pi}\left(Q^{2}\right) \\
& =2 C_{\pi} f_{\pi}\left\{3+\frac{\alpha_{S}\left(\mu_{R}^{2}\right)}{4 \pi}(-20)\right. \\
& \quad+\frac{\alpha_{S}^{2}\left(\mu_{R}^{2}\right)}{(4 \pi)^{2}}\left[\left(-\frac{2}{3} n_{f}\right)\left(-43.47-20 \ln \frac{\mu_{R}^{2}}{Q^{2}}\right)+\cdots\right] \\
& \quad+\cdots\} .
\end{aligned}
$$

The $n_{f}$-proportional NNLO contribution determines the value of the BLM scale

$$
\mu_{R}^{2}=\left(\mu_{B L M}^{2}\right)^{a s} \approx 0.114 Q^{2} \approx \frac{Q^{2}}{9} .
$$

One notes that this scale is considerably softer than the total momentum transfer $Q^{2}$, which is consistent with partitioning of $Q^{2}$ among the pion constituents. It should be pointed out, however, that in the $\overline{M S}$ scheme the BLM scale does not reflect the mean gluon momenta. Based on (7.8), the NLO prediction amounts to

$$
Q^{2} F_{\gamma^{*} \gamma \pi}\left(Q^{2}\right)=0.185+\frac{\alpha_{S}\left(\mu_{R}^{2}\right)}{\pi}(-0.309)+\cdots .
$$

This prediction obtained with the asymptotic distribution and the $\overline{M S}$ definition of the strong coupling renormalized at the BLM scale given by (7.9) is displayed in Fig. 9, along with the CLEO experimental data. For comparison also included in Fig. 9 is the NLO prediction obtained by employing the widely used choice $\mu_{R}^{2}=Q^{2}$. The usual one-loop formula for the QCD running coupling constant (C5) has been used with $\Lambda=\Lambda_{\overline{M S}}=0.2$ $\mathrm{GeV}^{2}$.
As it is seen from Fig. 9, the NLO results for the pion transition form factor display the following features. First, inclusion of the NLO contributions decreases the LO prediction. Second, predictions based on the asymptotic distribution are in reasonably good agreement with currently available experimental data.

In comparison with the choice $\mu_{R}^{2}=Q^{2}$, the BLM scale choice increases the absolute value of the ratio of the NLO to LO prediction by $\approx 11-6 \%$ for the values of $Q^{2}$ between 6 and $20 \mathrm{GeV}^{2}$. As an extension of the BLM scale-fixing prescription to all orders in perturbation theory, in [39] all the $\left(\beta_{0} \alpha_{s}\right)^{n}$ contributions to the pion transition form factor were resummed under the assumption of "Naive Non-Abelianization" (NNA). Our results cannot be directly compared with [39], but there are indications that the usual BLM scale-setting overestimates the size of higher-order contributions associated with the one-loop running coupling [40].

The complete NLO prediction for the pion transition form factor corresponding to the end-point concentrated $C Z$ distribution amplitude [37, given by Eqs. (7.4-7.6) with the coefficients $B_{2}=2 / 3$ and $B_{4}=0$, is shown in Fig. 10. Owing to the fact that the LO and NLO evolutional corrections to the $\mathrm{CZ}$ distribution are considerable [8], they have been taken into account. The BLM scale for the $\mathrm{CZ}$ distribution is higher than the BLM scale for the asymptotic DA, and it varies from $Q^{2} / 1.84$ to $Q^{2} / 2.37$ for $Q^{2}$ between 2 and $20 \mathrm{GeV}^{2}$.

As it is evident from Fig. 10, the complete NLO prediction for the pion transition form factor derived from the $\mathrm{CZ}$ distribution exceeds the experimental data significantly. This result can be considered as a serious failure of the $\mathrm{CZ}$ distribution amplitude. Comparing Figs. 9 and 10, one observes that the difference between our results for the asymptotic and CZ distribution amplitudes is sufficiently large for an unambiguous experimental discrimination between the two possibilities. Therefore, one expects that the pion distribution amplitude is closer to the asymptotic form than to the strongly end-point concentrated DA's like CZ 34 .

The size of higher-order QCD corrections represents the missing ingredient in assessing the validity of the perturbative prediction and the convergence of the expansion. One hopes that the BLM prescription offers a systematic way to choose the renormalization scale and minimize higher-order contributions. In order to check this for the case of the pion transition form factor, one would have to evaluate the complete NNLO contribution, which is a very demanding task. Another sensible indicator of the applicability of the perturbative calculation is the size of the expansion parameter $\alpha_{S}\left(\mu_{R}^{2}\right)$. The rather low BLM scale given in (7.9), and consequently the large $\alpha_{S}\left(\mu_{B L M}^{2}\right)$, questions the applicability of the perturbative prediction at experimentally accessible momentum transfers. Namely, the NLO predictions obtained in this paper assuming the asymptotic DA and the BLM scale (7.9) satisfy the requirement $\alpha_{S}\left(\mu_{R}^{2}\right)<0.5$ for $Q^{2} \geq 6$ $\mathrm{GeV}^{2}$. It should be pointed out, however, that there is 


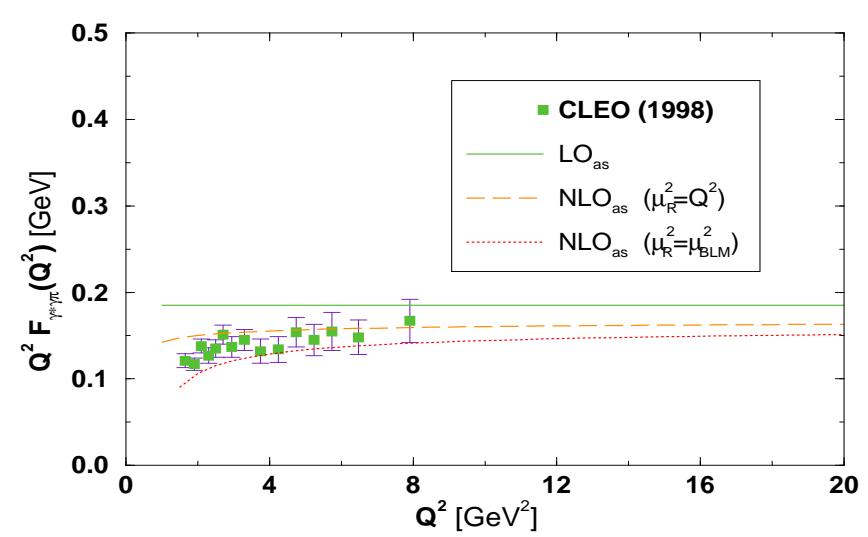

FIG. 9: The LO and NLO predictions for the pion transition form factor (scaled with $\left.Q^{2}\right) Q^{2} F_{\gamma^{*} \gamma \pi}\left(Q^{2}\right)$, obtained using the asymptotic DA. The NLO predictions are obtained using the BLM scale $\mu_{R}^{2}=\mu_{B L M}^{2} \approx Q^{2} / 9 \sqrt{7.9}$ and the commonly used choice $\mu_{R}^{2}=Q^{2}$. The experimental data are taken from [36.

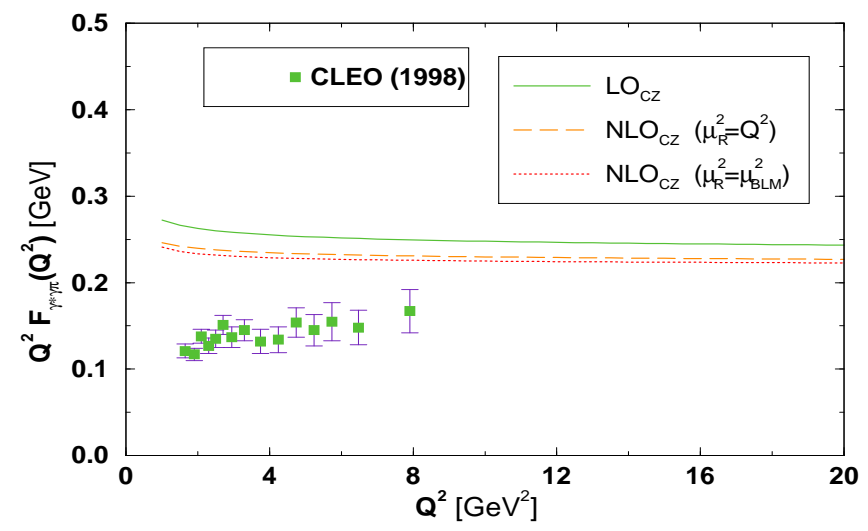

FIG. 10: The LO and NLO predictions for the pion transition form factor (scaled with $\left.Q^{2}\right) Q^{2} F_{\gamma^{*} \gamma \pi}\left(Q^{2}\right)$, obtained using the $\mathrm{CZ}$ distribution amplitude. The NLO predictions are obtained using the BLM scale determined from (7.7), and the commonly used choice $\mu_{R}^{2}=Q^{2}$. The experimental data are taken from 36.

an intrinsic disadvantage in using the $\overline{M S}$ running coupling (given by $(\overline{\mathrm{C} 5})$ ) as an expansion parameter, since it has a simple pole at $\mu_{R}^{2}=\Lambda^{2}$. This does not reflect the nonperturbative behavior of $\alpha_{S}\left(\mu_{R}^{2}\right)$ for small $\mu_{R}^{2}$, and a number of proposals have been suggested for the form of the coupling constant in this regime 41, 42, but its implementation demands caution [8]. For a recent application of 42 to the calculation of the pion transition form factor, see 43 .

So far nothing has been said concerning the renormalization scheme dependence of the predictions. It is known that the renormalization scheme dependence can be avoided by considering relations between physical observables, which must be independent of the choice of

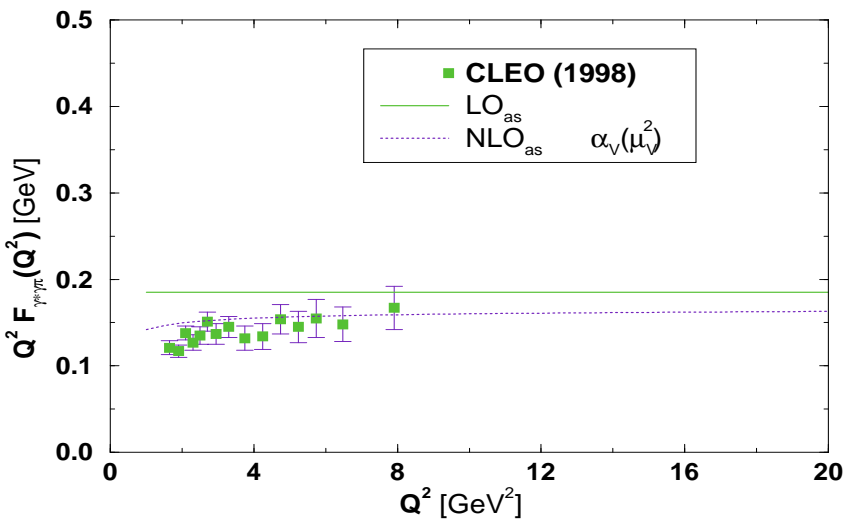

FIG. 11: The LO and NLO predictions for the pion transition form factor (scaled with $\left.Q^{2}\right) Q^{2} F_{\gamma^{*} \gamma \pi}\left(Q^{2}\right)$, obtained corresponding to the asymptotic DA in the $\alpha_{V}$ scheme with $\mu_{V}^{2} \approx Q^{2} / 1.7$ (7.14). The experimental data are taken from 36.

the scheme and scale to any fixed order of perturbation theory. This requirement can be expressed in the form of "commensurate scale relations" (CSR), in which the BLM scale-setting method is used to fix the renormalization scale [14]. In practice, a CSR for two observables is obtained by relating their respective perturbative predictions calculated in, for example, the $\overline{M S}$ scheme, and then by algebraically eliminating $\alpha_{\overline{M S}}$. The choice of the BLM scale ensures that the resulting CSR is independent of the choice of the intermediate renormalization scheme. Following this approach, in $[15]$ the exclusive hadronic amplitudes were calculated in the $\alpha_{V}$ scheme, in which the effective coupling $\alpha_{V}\left(\mu^{2}\right)$ is defined from the heavy-quark potential $V\left(\mu^{2}\right)$. The $\alpha_{V}$ scheme is a natural, physically based scheme, which by definition automatically incorporates vacuum polarization effects into the coupling. The $\mu_{V}^{2}$ scale which then appears in the $\alpha_{V}$ coupling reflects the mean virtuality of the exchanged gluons.

If use is made of the scale-fixed relation between the couplings $\alpha_{\overline{M S}}$ and $\alpha_{V}$ 15

$$
\alpha \overline{M S}\left(e^{-5 / 3} \mu_{V}^{2}\right)=\alpha_{V}\left(\mu_{V}^{2}\right)\left(1+\frac{\alpha_{V}\left(\mu_{V}^{2}\right)}{4 \pi} \frac{8 C_{A}}{3}+\cdots\right),
$$

then, to the order we are calculating, the prediction for the pion transition form factor takes the form

$$
F_{\gamma^{*} \gamma \pi}\left(Q^{2}\right)=F_{\gamma^{*} \gamma \pi}^{(0)}\left(Q^{2}\right)+\frac{\alpha_{V}\left(\mu_{V}^{2}\right)}{4 \pi} F_{\gamma^{*} \gamma \pi}^{(1)}\left(Q^{2}\right)+\cdots,
$$

with the "V" scale being given by

$$
\mu_{V}^{2}=e^{5 / 3} \mu_{B L M}^{2} .
$$

Now, on the basis of (7.9) and (7.13) one finds that the $V$ scale, corresponding to the asymptotic distribution and 
reflecting the mean (NLO) gluon momentum, is

$$
\left(\mu_{V}^{2}\right)^{a s} \approx 0.6025 Q^{2} \approx \frac{Q^{2}}{1.7} .
$$

Just for comparison, it is worth mentioning here that the corresponding $\mu_{V}^{2}$ scale for the pion electromagnetic form factor amounts to $Q^{2} / 20$ [8, 15].

Furthermore, since $\alpha_{V}$ is an effective running coupling defined from the physical observable it must be finite at low momenta, and the appropriate parameterization of the low-energy region should in principle be included. Nevertheless, in the energy region we are interested in, the usual one-loop conventional solution of the renormalization group equation for the QCD coupling $(\overline{C 5})$ can be employed. The numerical NLO prediction for the pion transition form factor obtained from Eqs. (7.12 7.14) and calculated with $\Lambda_{V}=0.16 \mathrm{GeV}^{2}$ is depicted in Fig. 11 . As can be seen, it is in good agreement with experimental data. The LO QCD correction, i.e., the NLO contribution, lowers the LO prediction for $\approx 16 \%$ for $Q^{2} \approx 6$ $\mathrm{GeV}^{2}$, i.e., for $\alpha_{V}\left(\mu_{V}^{2}\right) \approx 0.3$.

\section{SUMMARY AND CONCLUSIONS}

In this paper we have determined the NLO BrodskyLepage-Mackenzie (BLM) scale and obtained the complete NLO prediction for the pion transition form factor.

To determine the NLO BLM scale, a consistent and detailed calculation of both the hard-scattering and the perturbatively calculable part of the pion distribution amplitude has been performed up to $n_{f}$-proportional NNLO contributions. The calculation has been carried out in the Feynman gauge. To control both the UV and collinear divergences the dimensional regularization method has been employed. By combining, according to Eq. (3.13), and matching the results for the hard-scattering amplitude with the corresponding results obtained for the distribution amplitude, a proper cancellation of collinear singularities has been established and the $\gamma_{5}$ ambiguity problem (related to the use of dimensional regularization) has been resolved using the naive- $\gamma_{5}$ as well as the 't Hooft-Veltman (HV) schemes. As a result, the complete leading-twist NLO prediction for the pion transition form factor has been obtained as given by Eqs. $(6.22)$ and (7.4 (7.6), and Eqs. (7.2a) and (7.7), specifying the corresponding BLM scale. Derived in the $\overline{M S}$ scheme (for which suitable compact form (5.3) has been adopted), these expressions are valid for arbitrary form of the distribution amplitude (with the evolutional effects taken into account), and represent the main result of the present paper.

It has been demonstrated that the prediction for the leading-twist perturbative QCD prediction for the pion transition form factor is independent of the factorization scale $\mu_{F}^{2}$ at every order in the strong coupling constant $\alpha_{S}$ provided both the hard-scattering amplitude and the pion distribution amplitude are treated consistently regarding their $\mu_{F}^{2}$ dependence. The factorization scale disappears from the final prediction at every order in $\alpha_{S}$ without introducing any theoretical uncertainty. Consequently, for practical purposes the simplest and commonly used choice $\mu_{F}^{2}=Q^{2}$ is justified at the intermediate steps of the calculation.

Based on the general expressions (6.22) and (7.4 7.6.), the NLO predictions for the pion transition form factor have been obtained using the asymptotic and the $\mathrm{CZ}$ distribution amplitudes, with the renormalization scales being given by the respective BLM scales determined from Eqs. (7.2a) and (7.7). These predictions are shown in Figs. 9 and 10, respectively. By comparing these figures, one observes that, while, on one hand, the prediction derived from the asymptotic distribution is in good agreement with the presently available experimental data, on the other hand, the prediction obtained assuming the $\mathrm{CZ}$ distribution exceeds the data significantly, clearly demonstrating the inadequacy of the CZ distribution. This is in accordance with the conclusions reached in 32, 33, 34, 38], according to which the distribution amplitude is closer to the asymptotic form than to the end-point concentrated distribution of the CZ type. The renormalization scale $\mu_{R}^{2}$ fixed according to the BLM scale setting prescription within the $\overline{M S}$ scheme and corresponding to the asymptotic pion distribution amplitude, turns out to be $\mu_{R, B L M}^{2} \approx Q^{2} / 9$. Thus, in the region of $Q^{2}<8 \mathrm{GeV}^{2}$, in which the experimental data exist, $\mu_{B L M}^{2}<1 \mathrm{GeV}^{2}$. Consequently, the prediction obtained with $\mu_{R}^{2}=\mu_{B L M}^{2}$ cannot, in this region, be considered reliable.

In addition to the results calculated with the asymptotic distribution and $\overline{M S}$ renormalization scheme, the numerical prediction assuming the same distribution but in the $\alpha_{V}$ scheme, with the renormalization scale $\mu_{R}^{2}=$ $\mu_{V}^{2}=e^{5 / 3} \mu_{B L M}^{2} \approx Q^{2} / 2$, has also been obtained. It is displayed in Fig. 11 and as seen, is in good agreement with experimental data. Due to the fact that the scale $\mu_{V}^{2}$ reflects the mean gluon momentum in the NLO diagrams, it is to be expected that the higher-order QCD corrections are minimized, so that the leading order QCD term gives a good approximation to the complete sum.

\section{Acknowledgments}

One of us (B.M.) acknowledges the support by the Alexander von Humboldt Foundation and the hospitality of the theory groups at the Institut für Physik, Universität Mainz \& Institut für Theoretische Physik, Universität Würzburg. This work was supported by the Ministry of Science and Technology of the Republic of Croatia under Contract No. 00980102. 


\section{APPENDIX A: $\gamma_{5}$ PROBLEM}

\section{General remarks}

When using dimensional regularization, one runs into trouble with quantities that have the well-defined properties only in $D=4$ space-time dimensions, that is, with the Levi-Civita tensor $\epsilon_{\mu \nu \lambda \kappa}$, which is a genuine 4 dimensional object, and consequently with the pseudoscalar $\gamma_{5}$ Dirac matrix. The generalization of the $\gamma_{5}$ matrix in $D$ dimensions represents a problem, since it is not possible to simultaneously retain its anticommuting and trace properties. In practice, the ambiguity arises when evaluating a trace containing a $\gamma_{5}$ and pairs of contracted $\gamma$ matrices and/or pairs of Dirac slashed loop momenta ${ }^{5}$.

To deal with a $\gamma_{5}$ matrix, several possible schemes have been proposed in the literature. Following the previous calculation of the pion transition form factor [10], we have compared two of them in the present calculation.

In the so-called naive- $\gamma_{5}$ scheme [16], the anticommutation property of $\gamma_{5}$

$$
\left\{\gamma_{\mu}, \gamma_{5}\right\}=0
$$

is retained, while the cyclicity of the trace is abandoned, so that, for example,

$$
\begin{aligned}
& \operatorname{Tr}\left[\gamma_{5} \not d \gamma_{\mu} \not \phi \not \phi \not \gamma^{\mu}\right]=(D-6) \operatorname{Tr}\left[\gamma_{5} \not \subset \not b \not \phi d\right],(\mathrm{A} 2 \mathrm{a}) \\
& \operatorname{Tr}\left[\gamma^{\mu} \gamma_{5} \not d \gamma_{\mu} \not \partial \not \phi \not d\right]=(2-D) \operatorname{Tr}\left[\gamma_{5} \not \subset \not b \not \phi \not d\right] .(\mathrm{A} 2 \mathrm{~b})
\end{aligned}
$$

The traces obtained by cyclic permutation of the matrices $\gamma_{5}, \not \subset, \gamma_{\mu}, \not h, \not, \not,, \gamma^{\mu}$ can be divided into two classes, depending on the location of $\gamma_{5}$ with respect to the contracted $\gamma$ matrices: those in which $\gamma_{5}$ is outside the contracted pair as in (A2a), and those where $\gamma_{\mathrm{s}}$ are contracted through $\gamma_{5}$ as in (A2b). As is seen, the result (A2a) and the result (A2b), in which the anticommuting property (A1) of $\gamma_{5}$ had to be used before the contraction of $\gamma$ matrices can be performed, differ by $D-4$. Consequently, if the trace is multiplied by a pole in $D-4$, there appears a finite ambiguity in the result.

An alternative scheme has been proposed in the original paper on the dimensional regularization by 't Hooft and Veltman [17], and further systematized by Breitenlohner and Maison 18]. In this scheme, to which we refer as $\mathrm{HV}$ scheme, the anticommutativity of $\gamma_{5}$ is abandoned

5 The presence of a pair of Dirac slashed loop momenta leads in fact to the appearance of a pair of contracted $\gamma$ matrices, since the loop integration

$$
\int \frac{d^{D} l}{(2 \pi)^{D}} \frac{l^{\kappa} l^{\tau}}{(\text { Denominator })}=g^{\kappa \tau} I_{g}+\cdots
$$

transforms $l^{\kappa} l^{\tau} \gamma_{\kappa} \cdots \gamma_{\tau}$ into $\gamma_{\kappa} \cdots \gamma^{\kappa} I_{g}+\cdots$. Apart from the contracting $\gamma$ matrices and pairs of Dirac slashed loop momenta, the rest of the trace elements could be treated as 4 dimensional, so their (anti)commutation with $\gamma_{5}$ does not make a difference. and replaced by

$$
\begin{aligned}
\left\{\gamma_{\mu}, \gamma_{5}\right\} & =0 \text { for } \mu=0, \cdots, 4 \\
{\left[\gamma_{\mu}, \gamma_{5}\right] } & =0 \text { for } \mu=4, \cdots, D .
\end{aligned}
$$

For calculational purposes, it proves useful to introduce the following notation [18]:

$$
\begin{aligned}
g_{\mu \nu} & =\hat{\hat{g}}_{\mu \nu}+\hat{g}_{\mu \nu}, \\
\gamma_{\mu} & =\hat{\hat{\gamma}}_{\mu}+\hat{\gamma}_{\mu}, \\
l_{\mu} & =\hat{\hat{l}}_{\mu}+\hat{l}_{\mu},
\end{aligned}
$$

where

$$
\hat{\hat{g}}_{\mu}^{\mu}=4, \quad \hat{g}_{\mu}^{\mu}=D-4,
$$

and

$$
g_{\mu \kappa} \hat{g}_{\nu}^{\kappa}=\hat{g}_{\mu \nu}, \quad \hat{g}_{\mu \nu} \gamma^{\nu}\left(l^{\nu}\right)=\hat{\gamma}_{\mu}\left(\hat{l}_{\mu}\right) .
$$

The relation (A3) can then be written as

$$
\gamma_{\mu} \gamma_{5}=-\gamma_{5} \hat{\hat{\gamma}}_{\mu}+\gamma_{5} \hat{\gamma}_{\mu}
$$

This prescription for $\gamma_{5}$ violates the Ward identities and introduces "spurious" anomalies which violate chiral symmetry. To restore the Ward identities, finite counterterms should be added order by order in perturbation theory 26. In this scheme, the cyclicity of the trace is retained and the traces given in (A2) become

$$
\begin{aligned}
\operatorname{Tr}\left[\gamma_{5} \not d \gamma_{\mu} \not \partial \not \phi \not \gamma^{\mu}\right] & =\operatorname{Tr}\left[\gamma^{\mu} \gamma_{5} \not d \gamma_{\mu} \not \phi \not \phi \not d\right] \\
& =(D-6) \operatorname{Tr}\left[\gamma_{5} \not \phi \not \phi \not \phi d\right]
\end{aligned}
$$

As is seen, the result (A8), obtained in the $\mathrm{HV}$ scheme, corresponds to the result (A2a), obtained using the naive$\gamma_{5}$ scheme $^{6}$.

If a trace contains an even number of $\gamma_{5}$ matrices, then the property $\gamma_{5}^{2}=1$ can be used to eliminate $\gamma_{5}$ 's from the trace, and the Ward identities are preserved if the naive- $\gamma_{5}$ scheme (A1) is used 16 (the cyclicity of the trace is restored and the corresponding results are unambiguous). On the other hand, in the HV scheme the "spurious" anomalies can occur owing to the nonanticommuting property of $\gamma_{5}{ }^{7}$.

As for the traces containing an odd number of $\gamma_{5}$ matrices, we are left with the above mentioned ambiguities in the results.

6 As stated in [17], should we allow to anticommute $\gamma_{5}$ before continuation to $\vec{D}$ dimensions and after that use the HV scheme defined by A3), different results would emerge and ambiguity would reappear. We would again obtain two classes of results: the result A\& for $\gamma_{5}$ outside the contracting pair of $\gamma$ matrices, and the result $\mathrm{A} 2 \mathrm{~b}$ ) for anticommuting the matrix $\gamma_{5}$ in between the contracting $\gamma \mathrm{s}$. In this sense, the HV scheme would also lead to ambiguous results.

7 Again, should we allow to anticommute $\gamma_{5}$ before continuing to $D$ dimensions and then to use the HV scheme, different classes of results could be obtained, depending on the position of $\gamma_{5} \mathrm{~s}$. Hence, in this scheme, the ambiguity would still be present. 


\section{The $\gamma_{5}$ ambiguity in the $\gamma^{*} \gamma \rightarrow q \bar{q}$ amplitude}

There are two approaches one can take in order to resolve the $\gamma_{5}$ ambiguity problem in practical calculations (such as the calculation of the hard-scattering amplitude of the pion transition form factor). For each diagram, one can determine the way of manipulating the $\gamma_{5}$ matrices so that the Ward identities are preserved [9, 10]. Alternatively, one can perform the calculation using the $\mathrm{HV}$ scheme, and then introduce an additional renormalization constant which eliminates the "spurious" anomalies introduced by this prescription. In [10], the counterterm at the NLO order was calculated for the special case of hard-scattering amplitude already convoluted with the asymptotic distribution amplitude.

We have calculated the LO, NLO, and $n_{f}$-proportional NNLO terms to the hard-scattering amplitude using both the naive and the HV prescription for $\gamma_{5}$. By combining these results with the results for the distribution amplitude obtained in the same order, ambiguity in the naive$\gamma_{5}$ scheme has been resolved and the HV renormalization constant determined.

Although our end NLO result for the hard-scattering amplitude agrees with the result given in [10] (determined up to $\left.O\left(\epsilon^{0}\right)\right)$, the same is not true for the contributions of the individual one-loop diagrams of Fig. A when calculated using the naive prescription for $\gamma_{5}$.

Namely, owing to the fact that the relative position of $\gamma_{5}$ with respect to the Dirac slashed loop momenta was ignored, classes of terms appearing in the contribution of individual diagrams were omitted in [10].

For example, the trace corresponding to the diagram A23 of Fig. A leads to three different results depending on the position of $\gamma_{5}$ :

$$
\begin{aligned}
& \operatorname{Tr}\left[\gamma_{5} \not p \gamma^{\mu}(x p-\not q) \gamma_{\lambda}(x \not p-\not q-\not l) \gamma^{\nu}(-(1-x) p-\not h) \gamma^{\lambda}\right] \\
& \neq \operatorname{Tr}\left[\gamma^{\lambda} \gamma_{5} P \gamma^{\mu}(x \not P-\not q) \gamma_{\lambda}(x \not P-\not q-\not h) \gamma^{\nu}(-(1-x) P-\not l)\right] \\
& \neq \operatorname{Tr}\left[(-(1-x) p-\not l) \gamma^{\lambda} \gamma_{5} p \gamma^{\mu}(x p-\not q) \gamma_{\lambda}(x p-\not q-\not l) \gamma^{\nu}\right] \text {. }
\end{aligned}
$$

In Ref. [10], however, only the results for traces (A9a) and (A9b) were given. To conveniently describe the $\gamma_{5}$ ambiguity present in the contributions of $A 23$ and other diagrams, we have introduced the parameters $\delta$ and $\delta^{\prime}$. Thus, $\delta=0$ corresponds to the situation where $\gamma_{5}$ lies outside the contracting $\gamma \mathrm{s}$ and pairs of $/ \mathrm{s}$, as in (A9a). Corresponding to the case when $\gamma$ 's are con- tracted through the $\gamma_{5}$ matrix is $\delta=1$. For $\gamma_{5}$ not being placed between the pairs of $/ \mathrm{s}$, as in (A9b), $\delta^{\prime}=0$, while for $\gamma_{5}$ placed between the pairs $\Lambda \mathrm{s}$, as in (A9d), $\delta^{\prime}=1$. The contributions obtained using the HV scheme correspond to $\delta=0$. The contributions defined in 4.6a) of the individual NLO diagrams of Fig. 4 can then be parameterized by

$$
\begin{aligned}
\widetilde{T}_{A 11(22)}= & {\left[-\frac{1}{2}\left(\left[\eta_{U V}+2 \delta\right]-1\right)-\frac{1}{2}\left(\left[\eta_{I R}-2 \delta\right]+1\right)\right]+O(\epsilon) } \\
\widetilde{T}_{A 33}= & {\left[-\left[\eta_{U V}+2 \delta\right]+\ln (1-u)-1\right]+O(\epsilon) } \\
\widetilde{T}_{A 23}= & {\left[\left[\eta_{U V}+2 \delta\left(1+\delta^{\prime}\right)\right]+2 \eta_{I R}+\ln (1-u)-4\right]+O(\epsilon) } \\
\widetilde{T}_{A 13}= & {\left[\left[\eta_{U V}+2 \delta\left(1+\delta^{\prime}\right)\right]+2 \eta_{I R}\left(1+\frac{1}{u} \ln (1-u)\right)\right.} \\
& \left.+\frac{1}{u} \ln ^{2}(1-u)-\frac{3-u}{u} \ln (1-u)-4\right]+O(\epsilon) \\
\widetilde{T}_{A 12}= & {\left[-2 \frac{1-u}{u} \ln (1-u)\left[\eta_{I R}+2 \delta\left(1+\delta^{\prime}\right)\right]\right.} \\
& \left.-\frac{1-u}{u} \ln ^{2}(1-u)+10 \frac{1-u}{u} \ln (1-u)\right]+O(\epsilon),
\end{aligned}
$$

where to facilitate the comparison with the results of Ref. $\quad$ [10], we have introduced $\eta_{U V}=1 / \hat{\epsilon}+\ln \mu^{2} / Q^{2}, \eta_{I R}=$ 
$-1 / \hat{\epsilon}-\ln \mu^{2} / Q^{2}$, and $1 / \hat{\epsilon}=1 / \epsilon-\gamma+\ln (4 \pi)$. In 10 the results corresponding to $\delta^{\prime}=1$ in (A12) and (A13), as well as to $\delta^{\prime}=0$ in (A14), were omitted.

It was argued in 10 that since the quark propagator and the photon vertex corrections were related by the Ward identity of QED, they should be calculated as if they were not part of the trace with $\gamma_{5}$. This then determines the choice $\delta=0$ in diagrams $A 11, A 22, A 33$, $A 23$, and $A 13$. The remaining ambiguity associated with the IR (collinear) pole in diagram $A 12$ was resolved by repeating the calculation in the "equal-mass regularization", in which the quark and the gluon were given the same small mass $m$. It was found that the correct choice for diagram $A 12$ corresponded to the result obtained by contracting the $\gamma_{\mathrm{s}}$ through $\gamma_{5}$, and for $\gamma_{5}$ placed between the $\ell_{\mathrm{s}}$ (although the latter requirement was not stressed in 10), that is, for $\delta=1$ and $\delta^{\prime}=1$.

We have confirmed these choices by our calculation. In presenting our results in Sec. IV, we have already adopted the $\delta=0$ choice (or, equivalently, the HV results) for diagrams $A 11, A 22, A 33, A 23$, and $A 13$, as well as for the diagrams obtained from these by inserting the quark vacuum polarization loop. The ambiguity present in diagram $A 12$ and corresponding vacuum polarization diagram, is parameterized only by $\delta$, with $\delta^{6}$ taken as 1 .

A remark concerning the presentation of the $\gamma^{*} \gamma \rightarrow \bar{q} q$ amplitude in the form given by (2.1) is in order.

The multiplication of the amplitude by the factor $1 /\left(i e^{2}\right) N \epsilon_{\mu \nu \alpha^{\prime} \beta^{\prime}} P^{\alpha^{\prime}} q^{\beta^{\prime}}$ and the contraction of the LeviCivita tensors before the loop integrals are evaluated simplifies the calculation (since then at most three-point Feynman integrals appear). Since the generalization of the Levi-Civita tensors to $D$ dimensions is not unique, caution should be exercised when contracting these tensors. When using the HV scheme, the relation 18]

$$
\epsilon^{\mu_{1} \mu_{2} \mu_{3} \mu_{4}} \epsilon_{\nu_{1} \nu_{2} \nu_{3} \nu_{4}}=-\sum_{\pi \in S_{4}} \operatorname{sign} \pi \prod_{i=1}^{4} \hat{\hat{g}}_{\nu_{\pi(i)}}^{\mu_{i}}
$$

should be employed, in which case $N=2 / Q^{4}$. Note that the loop integrals with $\hat{\hat{l}}$ terms in the numerator of the integrand will be encountered. Only the one-loop integrals containing $\hat{\hat{l}}^{2}$ terms are different from the corresponding counterparts with $l$ in place of $\hat{\hat{l}}$, while the presence of the $\hat{i}^{\mu_{1}} \ldots \hat{l}^{\mu_{n}}$ terms does not alter the usual results. When using the naive- $\gamma_{5}$ scheme, the $\hat{\hat{g}}_{\nu_{\pi(i)}}^{\mu_{i}}$ from A15 can be replaced by $g_{\nu_{\pi(i)}}^{\mu_{i}}$, and then $N=2 /((D-2)(D-3)) 2 / Q^{4}$. We have checked our results by evaluating the contributions with and without the Levi-Civita contraction. The results agree.

\section{APPENDIX B: FEYNMAN RULES FOR THE PERTURBATIVELY CALCULABLE PART OF THE DA}

In this section we list the Feynman rules for the $\tilde{\phi}(u, t)$ operator (3.6) rederived following [20].

In Section II the distribution amplitude $\tilde{\phi}(u, t)$ for a state composed of a free quark and antiquark has been introduced (3.6)

$$
\begin{aligned}
\tilde{\phi}(u, t)= & \int \frac{d z^{-}}{2 \pi} e^{i(u-(1-u)) z^{-} / 2} \\
& \times\left\langle 0\left|\bar{\Psi}(-z) \frac{\gamma^{+} \gamma_{5}}{2 \sqrt{2}} \Omega \Psi(z)\right| q \bar{q} ; t\right\rangle \frac{1}{\sqrt{N_{c}}} .
\end{aligned}
$$

with $z^{+}=z_{\perp}=0$. The quark and antiquark carry the momenta $t P$ and $(1-t) P$, respectively, and the frame in which $P^{+}=P^{0}+P^{3}=1, P^{-}=P^{0}-P^{3}=0, P_{\perp}=0$ has been chosen. The path-ordered factor $\Omega(3.3)$

$$
\Omega=\exp \left\{i g \int_{-1}^{1} d s A^{+}(z s) z^{-} / 2\right\}
$$

makes $\tilde{\phi}(u, t)$ gauge invariant, and it can be expanded in perturbation theory as

$$
\Omega=\sum_{n=0}^{\infty} \frac{(i g)^{n}}{n !} \int_{-1}^{1} \prod_{k}^{n} d s_{k} A^{+}\left(s_{k} z\right) z^{-} / 2 .
$$

The path-ordering is immaterial since $A^{+}$fields commute. In the light-cone gauge $\left(A^{+}=0\right)$, this operator is unity, but generally (for example, in the Feynman gauge we are using) introduces extra diagrams. The $n$-th order term in the (B1) series will correspond to diagrams with $n$ gluon lines attached to the operator vertex. By inserting the term

$$
\theta\left(1-s_{k}\right) \theta\left(1+s_{k}\right)=i \int \frac{d r}{2 \pi} e^{i r s_{k}} \frac{1}{r}\left(e^{-i r}-e^{i r}\right)
$$

the limits of the integration in $(\mathrm{B} 1)$ are changed, and the function $\tilde{\phi}(u, t)$ takes the form 


$$
\begin{aligned}
\tilde{\phi}(u, t)= & \int \frac{d z^{-}}{2 \pi} e^{i(u-(1-u)) z^{-} / 2} \\
& \times \sum_{n=0}^{\infty} \frac{(i g)^{n}}{n !} i \int \frac{d r}{2 \pi} \frac{1}{r}\left(e^{-i r}-e^{i r}\right) \int_{-\infty}^{\infty} d s_{1} \cdots d s_{n} e^{\imath r\left(s_{1}+\cdots+s_{n}\right)} \\
& \times\left\langle 0\left|\bar{\Psi}(-z) \frac{\gamma^{+} \gamma_{5}}{2 \sqrt{2}} A^{+}\left(s_{1} z\right) \cdots A^{+}\left(s_{n} z\right) \Psi(z)\right| q \bar{q}\right\rangle\left(\frac{z^{-}}{2}\right)^{n} \frac{1}{\sqrt{N_{c}}} .
\end{aligned}
$$

The fields in (B3), along with the standard quark-gluon interaction insertions, are contracted in all possible ways, yielding different Feynman diagrams contributions. Here we list the Feynman rules derived from (B3).

The operator $\langle 0| \bar{\Psi}(-z) \frac{\gamma^{+} \gamma_{5}}{2 \sqrt{2}} \Psi(z)$ from Eq. (B3) will be represented by the crossed circle $\otimes$ in Feynman diagrams. The $\otimes$ vertex has a quark line entering and leaving the $\otimes$ vertex, and an arbitrary number of attached gluon lines $\left(A^{+}\right.$field can be contracted only with $A^{-}$, so the gluon line does not re-enter the vertex).

The general form of the $\otimes$ vertex with $n$ gluons attached to it is given by

$$
\frac{\gamma^{+} \gamma_{5}}{2 \sqrt{2}} \int \frac{d z^{-}}{2 \pi} e^{i S z^{-} / 2} \prod_{j=1}^{n}(i g) \frac{i}{q_{j}^{+}}\left(1-e^{i z^{-} q_{j}^{+}}\right),
$$

where

$$
S=u-(1-u)-k_{1}^{+}-k_{2}^{+}-\sum_{j=1}^{n} q_{j}^{+},
$$

and $q_{1}, \cdots, q_{n}$ are 4-momenta of the gluons entering the circle, while $k_{1}$ and $k_{2}$ are the 4-momenta of the quarks entering and leaving the circle, respectively. The form given in [20] is slightly different and incomplete regarding the gluon 4-momenta sign convention. In the special case when there are no gluons attached to the $\otimes$ vertex, and the notation $k_{1}=k$ and $k_{2}=k-P$ is used, the expression (B4) takes the form

$$
\frac{\gamma^{+} \gamma_{5}}{2 \sqrt{2}} \delta\left(u-k^{+}\right)
$$

The gluon propagator for the gluon attached to the $\otimes$ vertex (stemming from the $A^{+} A_{\nu}$ contraction) takes the form

$$
-i \frac{g_{\nu}^{+}}{q^{2}+i \eta} \delta_{a b}
$$

so after the $g_{\nu}^{+} \gamma^{\nu}$ contraction, the gluon-quark vertex for a gluon attached to the $\otimes$ vertex becomes

$$
-i g \gamma^{+} \frac{\lambda_{a}}{2}
$$

We will not specify here the usual Feynman rules already used in the calculation of the hard-scattering amplitude

Let us just note that similarly to the calculation of the hard-scattering amplitude, the correct spin and parity state of the $q \bar{q}$ state has been projected by multiplying the diagrams by

$$
\frac{\gamma_{5} \gamma^{-}}{2 \sqrt{2}}=\frac{\gamma_{5} P}{\sqrt{2}}
$$

and taking the trace. The color singlet nature of the $q \bar{q}$ state is taken into account by including the factor

$$
\sum_{\alpha=1}^{3} \frac{\delta_{\alpha \beta}}{\sqrt{N_{c}}}
$$

and consequently the trace over color indices must be taken. The result should be multiplied by an extra factor $1 / \sqrt{N_{c}}$ (see Eq. (B3)), which takes into account that $\tilde{\phi}(u, t)$ is normalized to give the LO result $\delta(u-t)$ (i.e., normalized to $\mathbb{1})$. Finally, note that we are investigating the meson flavor nonsinglet distribution amplitude.

\section{APPENDIX C: ON THE COUPLING CONSTANT RENORMALIZATION}

In this section we briefly resume the relevant ingredients of the coupling constant renormalization, which are frequently obscured in practical applications found in the literature, and we also introduce our specific representation (5.3) for the $\overline{M S}$ renormalization used in this calculation.

\section{Coupling constant renormalization}

It is well known that in $D=4$ dimensions, the QCD coupling strength $g$ is a dimensionless quantity $[g]=M^{0}$ ( $M$ denotes the mass unit), while in $D=4-2 \epsilon$ dimensions $[g]=M^{\epsilon}$. Obviously, the dimension of the "bare" coupling constant $\alpha_{S}$, related to "bare" $g$ by $g^{2}=$ $4 \pi \alpha_{S}$, corresponds to $\left[\alpha_{S}\right]=M^{2 \epsilon}$. The renormalized coupling, i.e., the running coupling $\alpha_{S}\left(\mu^{2}\right)$, is, naturally, a dimensionless quantity and the coupling constant renormalization introduces the additional scale $\mu^{2}$, whose 
presence balances the dimensions in the renormalization equation $\alpha_{S} \mu^{-2 \epsilon}=\alpha_{S}\left(\mu^{2}\right) Z_{\alpha}$. The scale introduced by the renormalization of the coupling constant is often referred to as renormalization (or coupling constant) scale and denoted by $\mu_{R}^{2}$, while the renormalization of the coupling constant in the MS scheme (the simplest renormalization scheme) reads

$$
\alpha_{S}=\mu_{R}^{2 \epsilon} \alpha_{S}\left(\mu_{R}^{2}\right)\left(1-\frac{\alpha_{S}\left(\mu_{R}^{2}\right)}{4 \pi} \beta_{0} \frac{1}{\epsilon}+O\left(\alpha_{S}^{2}\right)\right),
$$

where $\beta_{0}=11-2 / 3 n_{f}$.

In practical calculations, the additional scale $\mu^{2}$ is often introduced before the coupling constant renormalization (the presence of $\mu^{2}$ in Feynman integrals is quite standard), which corresponds to introducing the dimensionless $\left(\left[\alpha_{S}\right]=M^{0}\right)$ "bare" coupling constant $\alpha_{S}$ related to $g$ by $g^{2}=4 \pi \alpha_{S} \mu^{2 \epsilon}$, and consequently $\alpha_{S} \sim \mu^{-2 \epsilon}$. The "bare" and renormalized coupling constants are then related by $\alpha_{S}=\alpha_{S}\left(\mu^{2}\right) Z_{\alpha}$, and, if we choose to renormalize the coupling at the renormalization scale $\mu_{R}^{2}$ different from the scale $\mu^{2}$ introduced by regularization, the renormalization in MS scheme reads

$$
\alpha_{S}=\left(\frac{\mu_{R}^{2}}{\mu^{2}}\right)^{\epsilon} \alpha_{S}\left(\mu_{R}^{2}\right)\left(1-\frac{\alpha_{S}\left(\mu_{R}^{2}\right)}{4 \pi} \beta_{0} \frac{1}{\epsilon}+O\left(\alpha_{S}^{2}\right)\right) .
$$

We adopt the latter definition of the "bare" coupling constant as dimensionless quantity, and neglect the $O\left(\alpha_{S}^{3}\right)$ terms in further considerations.

From (C2) one trivially obtains the scale changing relation

$$
\begin{aligned}
& \alpha_{S}\left(\mu^{2}\right) \\
& =\left(\frac{\mu_{R}^{2}}{\mu^{2}}\right)^{\epsilon} \alpha_{S}\left(\mu_{R}^{2}\right)\left[1+\frac{\alpha_{S}\left(\mu_{R}^{2}\right)}{4 \pi} \beta_{0} \frac{1}{\epsilon}\left(\left(\frac{\mu_{R}^{2}}{\mu^{2}}\right)^{\epsilon}-1\right)\right] \\
& =\alpha_{S}\left(\mu_{R}^{2}\right)\left(1+\frac{\alpha_{S}\left(\mu_{R}^{2}\right)}{4 \pi} \beta_{0} \ln \frac{\mu_{R}^{2}}{\mu^{2}}\right)+O(\epsilon), \quad(\mathrm{C} 3 \mathrm{~b})
\end{aligned}
$$

the $\beta$ function

$$
\begin{aligned}
\beta\left(\alpha_{S}\left(\mu^{2}\right), \epsilon\right) & =\mu^{2} \frac{\partial}{\partial \mu^{2}} \alpha_{S}\left(\mu^{2}\right) \\
& =-\epsilon \alpha_{S}\left(\mu^{2}\right)-\frac{\alpha_{S}^{2}\left(\mu^{2}\right)}{4 \pi} \beta_{0},
\end{aligned}
$$

and, consequently, the running coupling

$$
\alpha_{S}\left(\mu^{2}\right)=\frac{4 \pi}{\beta_{0}\left(\left(\frac{\mu^{2}}{\Lambda^{2}}\right)^{\epsilon}-1\right) \frac{1}{\epsilon}}=\frac{4 \pi}{\beta_{0} \ln \frac{\mu^{2}}{\Lambda^{2}}}+O(\epsilon) .
$$

It is safe to ignore the $O(\epsilon)$ terms in (다), since the expression for the running coupling is usually introduced after all singularities have been removed (by renormalization and/or factorization). Generally, one cannot neglect the $O(\epsilon)$ terms in (C2) or (C3) (neither in order $\alpha_{S}$ nor in $\alpha_{S}^{2}$ ), and the use of the compact forms (C2) and (C3a) is preferred until all singularities are removed ${ }^{8}$.

\section{Renormalization schemes}

Next we turn to the choice of the renormalization scheme and the representations of that choice.

One can introduce different renormalization schemes by modifying the renormalization constant $Z_{\alpha}$ to $\bar{Z}_{\alpha}$ as a function of

$$
f(\epsilon)=1+\epsilon f^{(1)}+\epsilon^{2} f^{(2)}+\cdots,
$$

where $f(\epsilon)$ defines the renormalization scheme choice, while $\alpha_{S}=\alpha_{S}\left(\mu^{2}\right) \bar{Z}_{\alpha}$. Equation (C2) is then generalized to

$$
\alpha_{S}=\left(\frac{\mu_{R}^{2}}{\mu^{2}}\right)^{\epsilon} \alpha_{S}\left(\mu_{R}^{2}\right)\left(1-\frac{\alpha_{S}\left(\mu_{R}^{2}\right)}{4 \pi} \beta_{0} f(\epsilon) \frac{1}{\epsilon}\right),
$$

and the choice $f(\epsilon)=1 \equiv f_{M S}(\epsilon)$ corresponds to the MS scheme. Equations (C3) get modified by $\beta_{0} \rightarrow$ $\beta_{0} f(\epsilon)$.

The $\overline{M S}$ scheme is defined by

$$
f_{\overline{M S}}(\epsilon)=1+\epsilon(-\gamma+\ln (4 \pi))+O\left(\epsilon^{2}\right),
$$

and, to the order we are calculating, the $O\left(\epsilon^{2}\right)$ terms are arbitrary. Different definitions can be found in the literature: the original definition of the $\overline{M S}$ scheme 30] $f_{\overline{M S}}(\epsilon)=1+\epsilon(-\gamma+\ln (4 \pi))$ or the choices $f_{\overline{M S}}(\epsilon)=$ $\exp (\epsilon(-\gamma+\ln 4 \pi))$ and $f_{\overline{M S}}(\epsilon)=(4 \pi)^{\epsilon} / \Gamma(1-\epsilon)$ (for example, 27, 28] and [29], respectively), which mimic the $\epsilon$ dependence of the $\gamma^{n}, \ln ^{n} 4 \pi,\left(\pi^{2}\right)^{n}$ proportional terms introduced by dimensional regularization. Although they are all valid choices leading to the same $(\overline{M S})$ result (after renormalization and factorization of singularities), they unnecessarily complicate the calculation, since the intermediate results should be expanded over $\epsilon$ and since

8 One of the motivations for this short summary on the coupling constant renormalization was the appearance of different forms of $\alpha_{S}$ renormalization equations in the literature. The quite often used form

$$
\alpha_{S}=\alpha_{S}\left(\mu_{R}^{2}\right)\left[1-\frac{\alpha_{S}\left(\mu_{R}^{2}\right)}{4 \pi} \beta_{0}\left(\frac{1}{\epsilon}-\ln \frac{\mu_{R}^{2}}{\mu^{2}}\right)\right]
$$

represents an "effective" expression, which can be strictly used only for calculations in which there are no singularities apart from those that get renormalized by this coupling constant renormalization. The curious looking $\alpha_{S}$ renormalization procedure given in, for example, 27, presumably represents an attempt to generalize the above given "effective" form to all orders in $\epsilon$. In the presence of additional UV or IR singularities, the terms containing both scales $\mu_{R}^{2}$ and $\mu^{2}$ remain after the $\alpha_{S}$ renormalization, which is clearly inconsistent. The final finite results are correct since these curious looking terms are moved to renormalization and/or factorization constants. 
they do not contain the $(\Gamma)$ functions originally introduced by dimensional regularization. A more appropriate choice would be the one that contains combinations of $\Gamma$ s that naturally emerge in the calculation. For this calculation, in which both UV and IR singularities were regularized by dimensional regularization, the appropriate choice is (see (5.1) and (5.4))

$$
f_{\overline{M S}}(\epsilon)=\epsilon \Gamma(\epsilon) \Gamma(1-\epsilon) \frac{\Gamma(1-\epsilon)}{\Gamma(1-2 \epsilon)}(4 \pi)^{\epsilon}=\epsilon \Gamma_{U V}^{(0)}(\epsilon),
$$

while, for example, for the calculation in which only UV singularities were regularized by dimensional regularization, the choice $f_{\overline{M S}}(\epsilon)=\epsilon \Gamma(\epsilon)$ would be appropriate.

Alternatively, we can represent the dependence of the coupling constant renormalization on the renormalization scheme by $\alpha_{S} f(\epsilon)=\alpha_{S}\left(\mu^{2}\right) Z_{\alpha}$ (see, for example, [28]). The generalization of (C2) is then given by

$$
\alpha_{S}=\left(\frac{\mu_{R}^{2}}{\mu^{2}}\right)^{\epsilon}(f(\epsilon))^{-1} \alpha_{S}\left(\mu_{R}^{2}\right)\left(1-\frac{\alpha_{S}\left(\mu_{R}^{2}\right)}{4 \pi} \beta_{0} \frac{1}{\epsilon}\right),
$$

and it represents a representation alternative to (C7). Equations (C3 C5) are valid for the (C10) representation.

Since $\beta_{0}$ (and $\beta_{1}$ ) does not dependent on the choice of the renormalization scheme, the renormalization scheme and scale dependence can be described, to the order we are calculating, only by one parameter, (for example, the scale). The renormalization scheme and the renormalization scale are treated on the same footing in representation ( $\mathrm{C} 10)$ and their equivalence is explicit. For example, by substituting $\mu_{R}^{2}=\tilde{\mu}_{R}^{2}(f(\epsilon))^{\frac{1}{\epsilon}}, \Lambda^{2}=\Lambda_{M S}^{2}(f(\epsilon))^{\frac{1}{\epsilon}}$ into ( $\mathrm{C} 10)$, the renormalization equation (C2) in the MS scheme is obtained.

The another advantage of the $(\mathrm{C} 10)$ representation is that after the coupling constant renormalization is performed, the dimensional parameter $\epsilon$ remains as the only artifact of dimensional regularization. Consequently, when using Eq. (C7), the renormalization and factorization constants $Z_{\mathcal{M}, \text { ren }}, Z_{\mathcal{M}, \text { col }}$ contain $\gamma^{n}, \ln ^{n} 4 \pi,\left(\pi^{2}\right)^{n}$ proportional terms apart from the $\frac{1}{\epsilon^{n}}$ poles. In contrast, when using Eq. (C10) $Z_{\mathcal{M}, \text { ren }}, Z_{\mathcal{M} \text {, col }}$ contain only simple $\frac{1}{\epsilon^{n}}$ poles.

In this work the representation (C10) along with the definition (C9) of the $\overline{M S}$ scheme is used.
[1] S. L. Adler, Phys. Rev. 177, 2426 (1969); J. S. Bell and R. Jackiw, Nuovo Cim. A 60, 47 (1969).

[2] G. P. Lepage and S. J. Brodsky, Phys. Rev. D 22, 2157 (1980).

[3] A. V. Radyushkin and R. T. Ruskov, Nucl. Phys. B481, 625 (1996).

[4] G. P. Lepage and S. J. Brodsky, Phys. Lett. B 87, 359 (1979); Phys. Rev. Lett. 43, 545 (1979) [Erratum-ibid. 43, 1625 (1979)]; A. V. Efremov and A. V. Radyushkin, Theor. Math. Phys. 42, 97 (1980) [Teor. Mat. Fiz. 42, 147 (1980)]; Phys. Lett. B 94, 245 (1980); A. Duncan and A. H. Mueller, Phys. Lett. B 90, 159 (1980); Phys. Rev. D 21, 1636 (1980).

[5] J. Botts and G. Sterman, Nucl. Phys. B325, 62 (1989); H. Li and G. Sterman, Nucl. Phys.B381, 129 (1992); D. Tung and H. Li, Chin. J. Phys. 35, 651 (1997).

[6] R. D. Field, R. Gupta, S. Otto and L. Chang, Nucl. Phys. B186, 429 (1981); F. M. Dittes and A. V. Radyushkin, Sov. J. Nucl. Phys. 34, 293 (1981) [Yad. Fiz. 34, 529 (1981)]. M. H. Sarmadi, Ph. D. thesis, University of Pittsburgh, 1982, UMI 83-18195; R. S. Khalmuradov and A. V. Radyushkin, Sov. J. Nucl. Phys. 42, 289 (1985) [Yad. Fiz. 42, 458 (1985)]; E. Braaten and S. Tse, Phys. Rev. D 35, 2255 (1987).

[7] E. P. Kadantseva, S. V. Mikhailov and A. V. Radyushkin, Yad. Fiz. 44, 507 (1986) [Sov. J. Nucl. Phys. 44, 326 (1986)].

[8] B. Melic, B. Nizic and K. Passek, Phys. Rev. D 60, 074004 (1999); FizikaB 8, 327 (1999); in Proc. of Joint INT/JLab Workshop, Newport News 1999, Exclusive and semi-exclusive processes at high momentum transfer, 279-286, hep-ph/9908510.

[9] F. del Aguila and M. K. Chase, Nucl. Phys. B193, 517 (1981).
[10] E. Braaten, Phys. Rev. D 28, 524 (1983).

[11] I. V. Musatov and A. V. Radyushkin, Phys. Rev. D 56, 2713 (1997).

[12] B. Nizic, Phys. Rev. D 35, 80 (1987).

[13] S. J. Brodsky, G. P. Lepage and P. B. Mackenzie, Phys. Rev. D 28, 228 (1983).

[14] S. J. Brodsky and H. J. Lu, Phys. Rev. D 51, 3652 (1995).

[15] S. J. Brodsky, C. Ji, A. Pang and D. G. Robertson, Phys. Rev. D 57, 245 (1998).

[16] M. Chanowitz, M. Furman and I. Hinchliffe, Nucl. Phys. B159, 225 (1979).

[17] G. 't Hooft and M. Veltman, Nucl. Phys. B44, 189 (1972).

[18] P. Breitenlohner and D. Maison, Commun. Math. Phys. 52, 39 (1977).

[19] F. M. Dittes and A. V. Radyushkin, Phys. Lett. B 134, 359 (1984). M. H. Sarmadi, Phys. Lett. B 143, 471 (1984).

[20] G. R. Katz, Phys. Rev. D 31, 652 (1985); Ph.D.Thesis, Cornell University, 1986, UMI 86-23193.

[21] S. V. Mikhailov and A. V. Radyushkin, Nucl. Phys. B254, 89 (1985).

[22] S. V. Mikhailov and A. V. Radyushkin, Nucl. Phys. B273, 297 (1986).

[23] D. Müller, Phys. Rev. D 49, 2525 (1994), Phys. Rev. D 51, 3855 (1995).

[24] S. J. Brodsky, P. Damgaard, Y. Frishman and G. P. Lepage, Phys. Rev. D 33, 1881 (1986).

[25] G. Curci, W. Furmanski and R. Petronzio, Nucl. Phys. B175, 27 (1980).

[26] G. Bonneau, Nucl. Phys. B171, 477 (1980); Phys. Lett. B 96, 147 (1980); Nucl. Phys. B177, 523 (1981).

[27] E. B. Zijlstra and W. L. van Neerven, Nucl. Phys. B383, 525 (1992); P. J. Rijken and W. L. van Neerven, Nucl. 
Phys. B523, 245 (1998).

[28] S. Catani, Phys. Lett. B 427, 161 (1998).

[29] Z. Kunszt, A. Signer and Z. Trocsanyi, Nucl. Phys. B411, 397 (1994).

[30] W. A. Bardeen, A. J. Buras, D. W. Duke and T. Muta, Phys. Rev. D 18, 3998 (1978).

[31] Y. Matiounine, J. Smith and W. L. van Neerven, Phys. Rev. D 58, 076002 (1998).

[32] V. Braun and I. Halperin, Phys. Lett. B 328, 457 (1994); R. Jakob, P. Kroll and M. Raulfs, J. Phys. G 22, 45 (1996).

[33] A. V. Radyushkin, Few Body Syst. Suppl 11, 57 (1999).

[34] A. V. Radyushkin, Acta Phys. Polon. B 26, 2067 (1995); S. Ong, Phys. Rev. D 52, 3111 (1995); P. Kroll and M. Raulfs, Phys. Lett. B 387, 848 (1996).

[35] A. P. Bakulev, A. V. Radyushkin and N. G. Stefanis, Phys. Rev. D 62, 113001 (2000).

[36] J. Gronberg et al. [CLEO Collaboration], Phys. Rev. D 57, 33 (1998).

[37] V. L. Chernyak and A. R. Zhitnitsky, Phys. Rept. 112,
173 (1984).

[38] A. Schmedding and O. Yakovlev, Phys. Rev. D 62, 116002 (2000); A. P. Bakulev, S. V. Mikhailov and N. G. Stefanis, Phys. Lett. B 508, 279 (2001).

[39] P. Gosdzinsky and N. Kivel, Nucl. Phys. B521, 274 (1998).

[40] M. Beneke and V. M. Braun, Phys. Lett. B 348, 513 (1995); P. Ball, M. Beneke and V. M. Braun, Nucl. Phys. B452, 563 (1995).

[41] J. M. Cornwall, Phys. Rev. D 26, 1453 (1982); A. Donnachie and P. V. Landshoff, Nucl. Phys. B311, 509 (1989).

[42] D. V. Shirkov and I. L. Solovtsov, JINR Rapid Commun. 2 [76] (1996) 5, hep-ph/9604363; Phys. Rev. Lett. 79, 1209 (1997); D. V. Shirkov, Nucl. Phys. Proc. Suppl. 64, 106 (1998).

[43] N. G. Stefanis, W. Schroers and H. C. Kim, Eur. Phys. J. C 18, 137 (2000). 